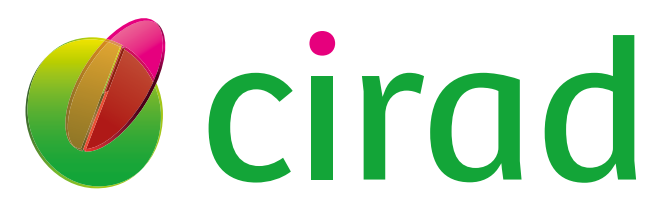

LARECHERCHE AGRONOMIQUE POUR LE DÉVELOPPEMENT

IMPRESS EX ANTE

\title{
UNE PROPOSITION DE DÉMARCHE POUR CONSTRUIRE EX ANTE LES CHEMINS DE L'IMPACT
}

Auteurs de l'équipe ImpresS impliqués dans l'élaboration et la rédaction de ce guide: Blundo Canto G. • Barret D. - Faure G. - Hainzelin E. - Monier C. • Triomphe B.

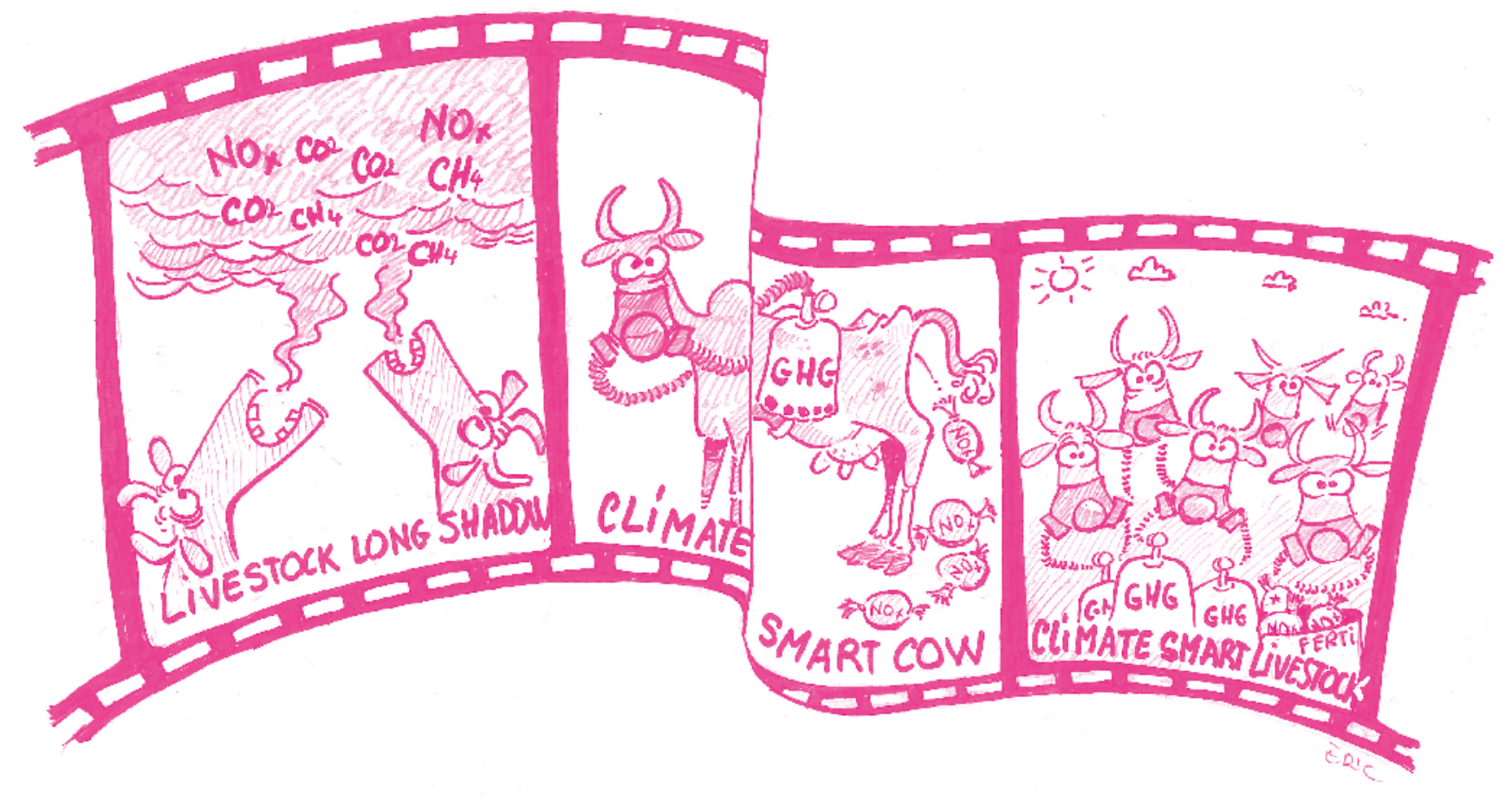



IMPRESS EX ANTE

UNE PROPOSITION DE DÉMARCHE POUR CONSTRUIRE EX ANTE LES CHEMINS DE L'IMPACT

Auteurs de l'équipe ImpresS impliqués dans l'élaboration et la rédaction de ce guide : Blundo Canto G. • Barret D. • Faure G. • Hainzelin E. • Monier C. • Triomphe B. 
(c) Cirad, janvier 2018

ISBN : 978-2-87614-734-8

https://doi.org/10.19182/agritrop/00010

Amélioration éditoriale : Sylvie Albert

Illustrations : Eric Vall, UMR Selmet, Cirad

Mise en page : Jean-Marie Forgue, Alter ego communication, 34150

Coordination : Patricia Doucet, délégation à la communication du Cirad

(C) Cette œuvre est mise à disposition selon les termes de la licence Creative Commons CC-BY 4.0 : Attribution 4.0 International https://creativecommons.org/licenses/by/4.0/deed.fr
Comment citer ce document

Blundo Canto G., Barret D., Faure G., Hainzelin E., Monier C., Triomphe B. Vall E. (illus.), 2018. ImpresS ex ante. Une proposition de démarche pour construire ex ante les chemins de l'impact. Montpellier, France : Cirad, 64 p. ISBN : 978-2-87614-734-8.

https://doi.org/10.19182/agritrop/00010. 


\section{Sommaire}

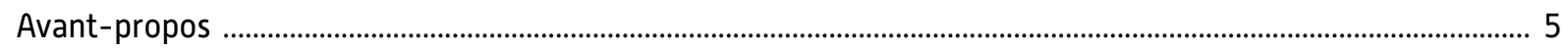

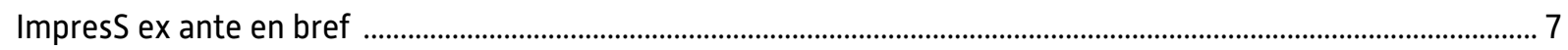

ImpresS ex ante : une démarche participative, itérative et adaptative en six étapes ............................................. 9

Introduction à la démarche ............................................................................................................................. 9

Les principes directeurs de la démarche ........................................................................................................ 11

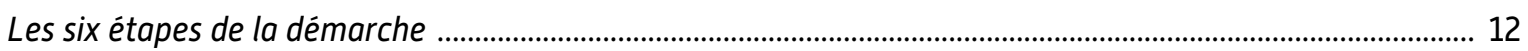

Une démarche adaptative, itérative et participative ........................................................................................ 13

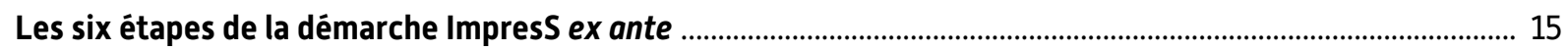

Étape 1: Construire le récit ........................................................................................................................ 15

Étape 2: Cartographier les résultats (outcomes] …………………………………………………………..... 28

Étape 3 : Prendre en compte les politiques publiques ...................................................................................... 35

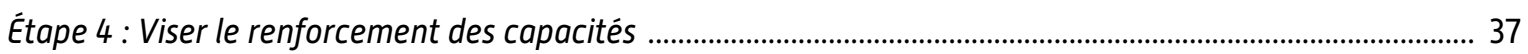

Étape 5 : Finaliser le chemin de l'impact et imaginer des chemins de l'impact alternatifs .............................. 39

Étape 6 : Suivi-évaluation participatif et apprentissage .................................................................................. 42

L'atelier ImpresS ex ante ……………………………………………………………………………………... 45

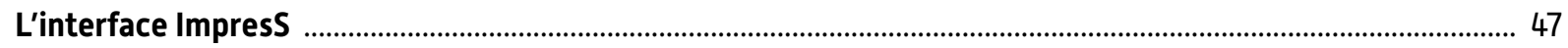

Adapter la démarche ImpresS ex ante à différente situations ............................................................................. 53

Adapter la démarche à différentes ressources disponibles .................................................................................. 53

Adapter le récit pour différentes audiences ........................................................................................................... 55

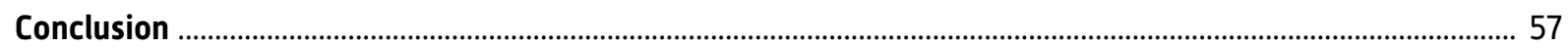

Liste des figures, tableaux, encadrés \& trucs et astuces ..................................................................................... 58

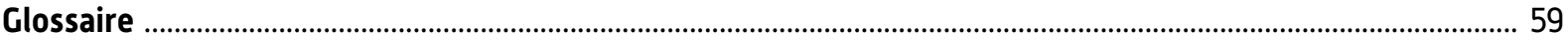

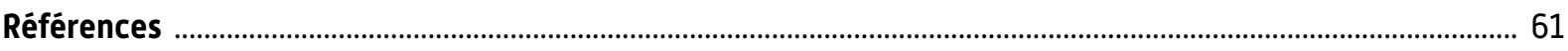

Annexe. Complémentarités et intégration avec la démarche projet et les outils classiques .............................. 62 



\section{Avant-propos}

Ce document est la première mise en forme d'une réflexion en cours sur la construction ex ante des chemins de l'impact d'une intervention de la recherche. Des améliorations concernant son utilisation dans différents contextes seront proposées sur la base de retours d'expérience ; ce n'est donc pas un guide définitif. II est utile pour des chercheurs ou des responsables engagés dans la recherche afin de construire des projets ou des programmes de recherche en s'intéressant avant tout aux transformations que peuvent provoquer des recherches conduites avec des acteurs. Il est aussi utile aux partenaires des chercheurs et aux chargés de valorisation qui accompagnent la formulation des projets et programmes ex ante. Ce guide est structuré de façon didactique, en précisant les étapes de la démarche proposée :

1. Construire le récit ;

2. Cartographier les résultats ;

3. Prendre en compte les politiques publiques ;

4. Viser le renforcement des capacités ;

5. Finaliser le chemin de l'impact et imaginer des chemins de l'impact alternatifs ;

6. Définir un système de suivi-évaluation participatif et d'apprentissage.

Tout au long du texte, des encadrés sont utilisés pour préciser des concepts, et des outils sont proposés pour faciliter la mise en œuvre de la méthode. Mais la méthode implique des allers-retours fréquents entre les étapes, car une réflexion menée à une étape donnée peut amener à modifier ce qui a été proposé dans une étape antérieure. Les textes en rouge indiquent les moments où de telles boucles sont nécessaires.

Ce symbole après le titre d'un paragraphe signifie qu'un travail est en cours pour approfondir et améliorer la méthode proposée dans ce paragraphe. 



\section{ImpresS ex ante en bref}

La recherche est de plus en plus appelée à documenter les impacts qu'elle contribue à générer ${ }^{1}$. Cela ne se limite pas à renseigner la section " impact " des appels à propositions et appels d'offres, mais doit amener à mieux positionner les propositions de recherche par rapport à leur capacité à répondre aux besoins sociétaux et environnementaux. Le Cirad a la volonté institutionnelle, nourrie par une histoire collective, de développer une culture de l'impact, tant au niveau individuel que collectif, se fondant sur une meilleure compréhension des mécanismes à l'œuvre dans les systèmes et processus d'innovation pour générer des impacts dans la durée.

Dans ce contexte, le présent document livre une réflexion structurée, dénommée démarche ImpresS ex ante, pour orienter et outiller la construction des chemins de l'impact ${ }^{2}$ au moment de concevoir une intervention de recherche ou de recherche-développement en partenariat ${ }^{3}$ et avant sa mise en œuvre. La démarche ImpresS ex ante a comme objectif principal d'aider les chercheurs du Cirad, leurs partenaires et les chargés de valorisation, à construire une vision partagée de ces interventions et des chemins de l'impact plausibles.

Cette démarche permet de nourrir une réflexion collective sur la place de la recherche dans l'émergence d'impacts sociétaux et environnementaux et sur la façon dont ses interactions avec divers acteurs génèrent des changements de pratiques et de comportements aboutissant à ces impacts. Elle est centrée sur les changements de perceptions, de pratiques et de comportements des acteurs que l'intervention souhaite ou peut provoquer à travers l'appropriation (incluant des transformations, adaptations ou rejets possibles) de ses produits et l'analyse des obstacles potentiels à cette appropriation, afın d'aboutir à des stratégies d'intervention cohérentes et plausibles pour surmonter ces derniers. Elle est donc dans l'idéal menée avec les acteurs majeurs impliqués dans une intervention.

La démarche présentée vise en outre à aider la recherche à mieux communiquer sur ses intentions d'interventions auprès de différents types d'acteurs. Elle permet de répondre de manière plus convaincante aux appels à propositions des bailleurs, notamment sur la question de la contribution aux impacts, qui est devenue un critère d'évaluation discriminant. Elle permet également l'élaboration à destination de la société civile, des tutelles et des partenaires, d'un discours rigoureux et étayé sur l'impact auquel contribuent les recherches, parfois sur des pas de temps très longs. Elle n'est pas normative; tout en étant complémentaire des démarches projet existantes, elle se veut souple et adaptative pour permettre aux équipes qui montent des interventions de s'adapter aux moyens dont elles disposent, aux temporalités et de répondre aux attentes des différents acteurs.

1 Voir à ce sujet https://sciencebusiness.net/news/question-impact

2 Le chemin de l'impact consiste en la description d'un processus d'innovation qui met en évidence des relations de causalité entre les moyens [inputs] mobilisés par l'intervention, les produits [outputs) de l'intervention, les résultats [outcomes], qui se matérialisent directement au niveau des utilisateurs des produits [outputs] de l'intervention, et les impacts de $1^{\mathrm{er}}$ et $2^{\mathrm{e}}$ niveaux.

3 Dans tout le texte, le terme " intervention » est utilisé comme un terme générique pour défınir un projet, un programme, une grappe de projets, etc. Ce choix est lié au fait que la démarche ex ante proposée est applicable à différents " grains » de projets mais aussi à différentes interventions de recherche ou de développement impliquant la recherche. 
La démarche ImpresS ex ante s'appuie sur trois principes centraux :

1. L'étape cruciale de la construction du chemin de l'impact est la génération des résultats [outcomes], qui consistent en une appropriation (utilisation, adoption, transformation, adaptation) par les acteurs des produits (outputs) d'une intervention, cette étape conduisant à des changements chez certains acteurs ;

2. Parce que l'impact se génère dans le temps long, il faut réfléchir au-delà de l'horizon d'un projet individuel isolé, et considérer des grappes de projets inscrits dans une trajectoire d'innovation ;

3. La démarche doit produire un récit hypothétique de l'impact, décrivant ex ante des chemins plausibles de l'impact. C'est lors de la mise en œuvre de l'intervention que s'ajusteront progressivement et se transformeront ces chemins de l'impact plausibles en chemins réels [avérés], que le suivi-évaluation permettra de documenter.

Cette démarche, participative et adaptative, est menée, dans l'idéal, selon un processus itératif en six étapes :

1) construire un premier récit hypothétique de l'impact,

2) cartographier les résultats désirés,

3) prendre en compte les politiques publiques,

4) renforcer les capacités,

5) finaliser le récit du chemin de l'impact et imaginer des chemins alternatifs, et

6) défınir un système de suivi-évaluation participatif et d'apprentissage.

Chaque étape enrichit les suivantes, mais le récit est alimenté tout au long de la réflexion par les résultats des autres étapes, à travers des boucles de feedback qui permettent d'aboutir à un récit cohérent et plausible du chemin de l'impact. 


\section{ImpresS ex ante : une démarche participative, itérative et adaptative en six étapes}

\section{Introduction à la démarche}

Depuis 2013, le Cirad a fait le choix de « dépasser la culture de la promesse » [Hainzelin et al., 2017) pour développer une culture de l'impact au sein de l'institution, en y associant ses partenaires. Cet effort a pris la forme d'une méthode d'évaluation de l'impact ex post baptisée ImpresS [IMPact des REchercheS au Sud / IMPact of RESearch in the South). Entre 2015 et 2016, le Cirad a testé la méthode ImpresS ex post sur 13 études de cas ex post représentant la diversité de ses activités et de ses résultats dans le monde.

Afin de poursuivre le développement de cette culture de l'impact au Cirad, et de l'outiller, l'équipe d'ImpresS a ensuite élaboré une démarche ex ante, qui a comme objectif principal d'aider les chercheurs du Cirad, leurs partenaires et les chargés de valorisation à construire une vision partagée des interventions qui impliquent la recherche et des chemins de l'impact plausibles ${ }^{4}$. Elle vise à améliorer concrètement cette construction par une conception stratégique et participative des interventions, en explicitant les mécanismes par lesquels la recherche et ses partenaires entendent contribuer à l'impact. Cela suppose, tant au niveau individuel que collectif, de bien comprendre les mécanismes à l'œuvre dans les processus d'innovation. Cette culture de l'impact s'incarne donc dans les apprentissages, les pratiques de recherche, et la capitalisation des expériences collectives, et permettra à terme d'augmenter la probabilité de générer des impacts recherchés dans la durée.

La démarche ImpresS ex ante permet de nourrir une réflexion collective sur la place de la recherche dans l'émergence des impacts sociétaux et environnementaux et sur sa contribution, par des interactions avec les acteurs, à des changements de perceptions, de pratiques et de comportements souhaitables pour déclencher ces impacts. Elle est fondée sur les leçons tirées des 13 études de cas, de la littérature relative à l'évaluation, de quatre ateliers ex ante regroupant les chercheurs du Cirad et leurs partenaires, et d'une école-chercheurs en juin 2017, au cours de laquelle neuf équipes projet ont testé la première version de la démarche. Ainsi, le présent document emprunte à la recherche-action en partenariat (Faure et al., 2010], à l'analyse participative du chemin de l'impact (Douthwaite et al., 2007), à la cartographie des outcomes [Earl et al., 2001], aux applications de la théorie du changement (Alvarez et al., 2014 ; Mayne, 2015], à l'analyse de pouvoir des acteurs (Mayers, 2005 ; Schiffer et Hauck, 2010) et enfin aux leçons tirées de la démarches ImpresS ex post [Faure et al., 2018 ; Temple et al., 2018].

Ce document livre une réflexion structurée pour orienter et outiller la construction des chemins de l'impact pendant la conception d'une intervention et avant son démarrage. Dans ce texte, le terme « intervention ", générique et englobant, est utilisé pour définir un projet, un programme, une grappe de projets, etc. Ce choix est lié au fait que la démarche ex ante proposée est applicable à différents types d'initiatives

\footnotetext{
4 La diversité des interventions menées par les équipes du Cirad amène parfois à construire des chemins de l'impact pour lesquels seulement certains produits (outputs) sont générés par la recherche. Dans le cas d'interventions multi-acteurs, il faut en toute rigueur tenir compte du fait que les produits de l'intervention peuvent être obtenus par la recherche ou par d'autres partenaires avec l'appui de la recherche. Suivant le contexte et les objectifs visés, les équipes peuvent décider de réfléchir à l'appropriation des seuls produits issus de la recherche ou d'élargir leur réflexion aux produits issus en collaboration avec d'autres acteurs.
} 
et à différents " grains ". Construire le chemin de l'impact (figure 1) permet de bien identifier (i) les produits ${ }^{5}$ de l'intervention [outputs], (ii) les résultats [outcomes] qui sont les changements de pratiques [agricoles ou managériales], d'organisation, de règles et de comportements (usage, adaptation, transformation] liés à l'appropriation de ces produits par les acteurs interagissant directement ou indirectement avec la recherche et enfin (iii) les impacts, ou les effets de cette appropriation sur les populations ou sur l'environnement, qu'ils soient positifs ou négatifs (anticipation des risques). La démarche ImpresS ex ante explicite donc la logique de l'intervention ${ }^{6}$ de la recherche : pourquoi la mise en œuvre de certaines actions peut-elle entraîner les résultats souhaités et souhaitables ?

Input

Les équipes de Rintervention ont mobilisé ces ressources.
Output

Pour générer ces produits de lintervention.

\section{Outcome}

Les acteurs se sont saisis des produits

et les ont transformbes pour se les approprier.
Impact 1

Ces résultats ont provoqué des impacts chez les acteurs qui interagissent directement ou indirectement avec Fintervention...

\section{Impact 2}

Ces résultats ont provoque chez les bénéficiaires indirects.

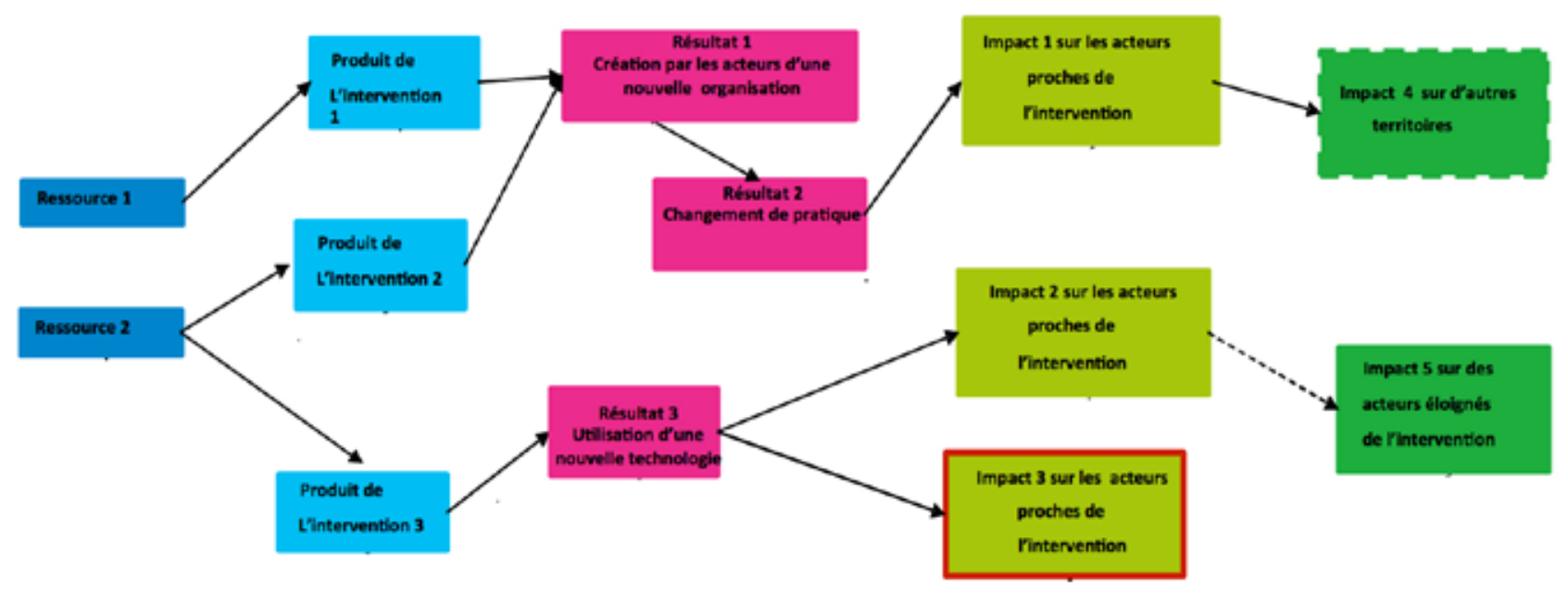

Figure 1 : Description générique d'un chemin de l'impact.

L'encadré 1 fournit la définition de quelques termes utilisés dans ce document.

5 Les produits d'une intervention peuvent être générés par la recherche « isolément " (par exemple un vaccin le ou contrôle biologique contre des ravageurs), mais ils peuvent aussi être générés par l'activité coordonnée de plusieurs acteurs (par exemple les schémas de sélection participative).

6 Ce document ne considère pas, de manière intentionnelle, les questions relatives à la définition de l'évaluation basée sur la (les) théorie[s] du (pour le] changement. L'expression " théorie du changement » peut prêter à une certaine confusion dans la langue française ; en effet, une théorie renvoie normalement à un « ensemble organisé de principes, de règles, de lois scientifiques visant à décrire et à expliquer un ensemble de faits ". Selon le dictionnaire Larousse, une théorie est aussi un " système d'hypothèses sous-tendant les interprétations des événements ". Cependant, la première définition est plus souvent utilisée et cette définition peut poser problème dans le cas de changements hypothétiques dans le futur. Pour éviter de rentrer dans des questions de sémantique, nous éviterons de disserter sur le concept de théorie du changement, même si, à l'évidence, la démarche ImpresS ex ante, qui traite des mécanismes hypothétiques par lesquels une intervention, et spécifiquement l'action de la recherche, contribue aux impacts, renvoie à cette notion telle qu'utilisée dans le monde anglo-saxon. 
Chemin de l'impact - Le chemin de l'impact consiste en la description d'un processus d'innovation qui met en évidence des relations de causalité entre les moyens [inputs] mobilisés par la recherche, les produits [outputs] de la recherche, les résultats [outcomes], qui se matérialisent directement au niveau des utilisateurs des produits (outputs) de la recherche, et les impacts de $1^{\text {er }}$ et $2^{\mathrm{e}}$ niveaux.

Moyen (input) de la recherche - L'input concerne l'ensemble des interventions et des ressources qui permettent de mener une activité de recherche (ressources humaines et matérielles, budget de recherche, informations, connaissances générées antérieurement ou tacites, autres activités de recherche, etc.) et ainsi de générer des produits (outputs) de recherche. Dans la méthode décrite dans ce guide, ces moyens (inputs) renvoient aux investissements réalisés et aux ressources mobilisées avant la période choisie pour le démarrage de l'étude de cas ou pendant la période de l'étude de cas (un financement ou le recrutement d'un chercheur peut intervenir pendant la période de l'étude de cas et reste un input].

Produit [output] de la recherche - L'output est la production issue de la recherche ou des interactions de la recherche avec les acteurs dans le[s] projet(s]. II peut s'agir de connaissance, scientifique ou non (publication, rapport, base de données, méthode, etc.), de formations professionnelles ou académiques, d'une expertise, d'une technologie, d'un réseau ou d'autres formes de production. Les outputs peuvent contribuer à l'émergence d'innovations lorsqu'ils font l'objet d'une appropriation par les acteurs de la société. Dans la méthode décrite dans ce guide, les produits de la recherche [connaissances, prototypes, etc.] élaborés avant le démarrage de l'étude de cas sont considérés comme des inputs alors que ceux élaborés pendant la période de l'étude de cas sont considérés comme des outputs.

Résultat [outcome] - L'outcome est l'appropriation d'un produit de la recherche par les acteurs interagissant directement ou indirectement avec la recherche, qui conduit à des changements de pratiques (agricoles ou managériales], des changements organisationnels, d'interactions ou de règles.

Impacts - Les impacts sont des effets à long terme, positifs et négatifs, intentionnels ou non, directs ou indirects, induits par une action de développement. Les impacts, c'est ce qui reste une fois que le projet / programme est terminé. Il peut s'agir d'impacts de différentes natures économiques, sociaux, territoriaux, environnementaux, politiques, sanitaires. Ils sont mesurés par des indicateurs.

Impacts de $\mathbf{1}^{\text {er }}$ niveau [ou impacts 1 ] - Les impacts de $1^{\text {er }}$ niveau sont mesurés sur les acteurs interagissant directement ou indirectement avec la recherche et/ou les acteurs majeurs de l'innovation, et peuvent être évalués avec ces acteurs. Leur mesure concerne deux critères: I'intensité du changement et l'ampleur du changement.

Impacts de $\mathbf{2}^{\mathrm{e}}$ niveau [ou impacts 2 ] - Les impacts de $\mathbf{2}^{\mathrm{e}}$ niveau résultent des effets de spillover (impacts indirects) ou de changement d'échelle (horizontal / scaling out et vertical / scaling up]. Ils peuvent être évalués selon les critères d'intensité du changement et d'ampleur du chan- gement, mais de manière plus approximative ou moins détaillée que les impacts de $1^{\text {er }}$ niveau.

À noter que les notions de moyens (inputs], de produits (outputs), de résultats (outcomes), et d'impacts de $1^{\text {er }}$ et 2 niveaux connaissent des interprétations différentes selon les disciplines, les auteurs, les institutions. En effet, certains auteurs décomposent parfois les outcomes en " outcomes intermédiaires " et " outcomes de long terme ". À l'inverse, d'autres ne décomposent pas les impacts en impacts de $1^{\mathrm{er}}$ et $2^{\mathrm{e}}$ niveaux. De plus, la distinction entre outputs et outcomes n'est pas toujours facile, notamment dans le cas de recherches participatives. Certains classent en outcomes ce que d'autres classent en outputs, ou inversement. De même, il n'est pas toujours facile de distinguer les outcomes des impacts, car un impact constaté au niveau d'un acteur peut devenir un outcome qui génèrera un impact pour un autre acteur qui interagit avec le premier.

Acteur - Un acteur est un individu ou une organisation impliqué[e] dans un processus d'innovation. Dans la démarche ImpresS ex post, trois catégories d'acteurs sont distinguées : les acteurs qui ont un rôle majeur dans le processus d'innovation, les acteurs qui influent (de manière intentionnelle ou non) sur l'innovation sans être acteurs du processus d'innovation et les acteurs qui sont impactés positivement ou négativement le cas échéant par I'innovation, mais qui ne sont pas des acteurs majeurs du processus d'innovation.

Descripteurs - Les descripteurs correspondent à l'expression de l'impact par les acteurs avec leurs propres mots et se référant à des changements tels qu'ils les perçoivent.

Indicateur - Un indicateur correspond à une information synthétique, de nature quantitative ou qualitative, qui permet de caractériser une ressource ou un processus ou d'aider à prendre une décision.

Radar - Un radar est une représentation graphique qui synthétise les données relatives aux différents impacts identifiés. La méthode ImpresS propose de représenter les impacts après les avoir regroupés en 11 " domaines d'impacts".

Récit de l'innovation - Ce récit est le narratif de l'innovation, donc de l'ensemble des acteurs majeurs de l'innovation. Il considère les grandes phases de l'histoire de l'innovation avec ses avancées, ses crises, les facteurs extérieurs qui influencent l'innovation. II s'agit de recenser et dater " ce qui s'est passé ", depuis les prémices du processus d'innovation jusqu'à la période actuelle.

Chronogramme - Un chronogramme permet de visualiser le récit de l'innovation dans sa dimension temporelle en précisant les évènements marquants.

Grappe de projets - Une grappe de projets est l'ensemble des projets de recherche, de recherche-développement et de développement, mais aussi des interventions non formalisées en projets, liés à l'innovation étudiée.

Intervention - Une intervention dans ce document définit soit un projet, soit un programme, soit une grappe de projets, la démarche ImpresS ex ante étant applicable à différents "grains " de projets, mais aussi à différentes interventions de recherche ou de développement impliquant la recherche. 


\section{Les principes directeurs de la démarche}

La démarche ImpresS ex ante s'appuie sur trois principes centraux :

1. L'étape cruciale de la construction du chemin de l'impact est la génération des résultats [outcomes], qui consistent en une appropriation (utilisation, adoption, transformation, adaptation) par les acteurs des produits (outputs) d'une intervention, cette étape conduisant à des changements chez certains acteurs ;

2. Parce que l'impact se génère dans le temps long, il faut réfléchir au-delà de l'horizon d'un projet individuel isolé, et considérer des grappes de projets inscrits dans une trajectoire d'innovation ;

3. La démarche doit produire un récit hypothétique de l'impact, décrivant ex ante des chemins plausibles de l'impact. C'est lors de la mise en œuvre de l'intervention que s'ajusteront progressivement et se transformeront ces chemins de l'impact plausibles en chemins réels (avérés], que le suivi-évaluation permettra de documenter.

La démarche ImpresS ex ante aide à (i) définir une programmation stratégique et la mise en œuvre de l'intervention en ciblant et en explicitant les processus qui produisent les résultats [outcomes], (ii) communiquer en interne et en externe sur les interventions de la recherche, et (iii) faciliter la conception d'un système de suivi-évaluation des actions pour mieux piloter la mise en œuvre de l'intervention, nourrir la réflexion collective et capitaliser les leçons apprises.

\section{Les six étapes de la démarche}

Une réflexion structurée en plusieurs étapes et ajustable en fonction des interventions est présentée dans ce document (figure 2). Chaque étape implique l'usage d'outils : certains sont discutés en tant que tels dans ce document, mais les équipes peuvent choisir d'autres outils existants qu'elles considèrent comme les plus pertinents pour aboutir au résultat recherché.

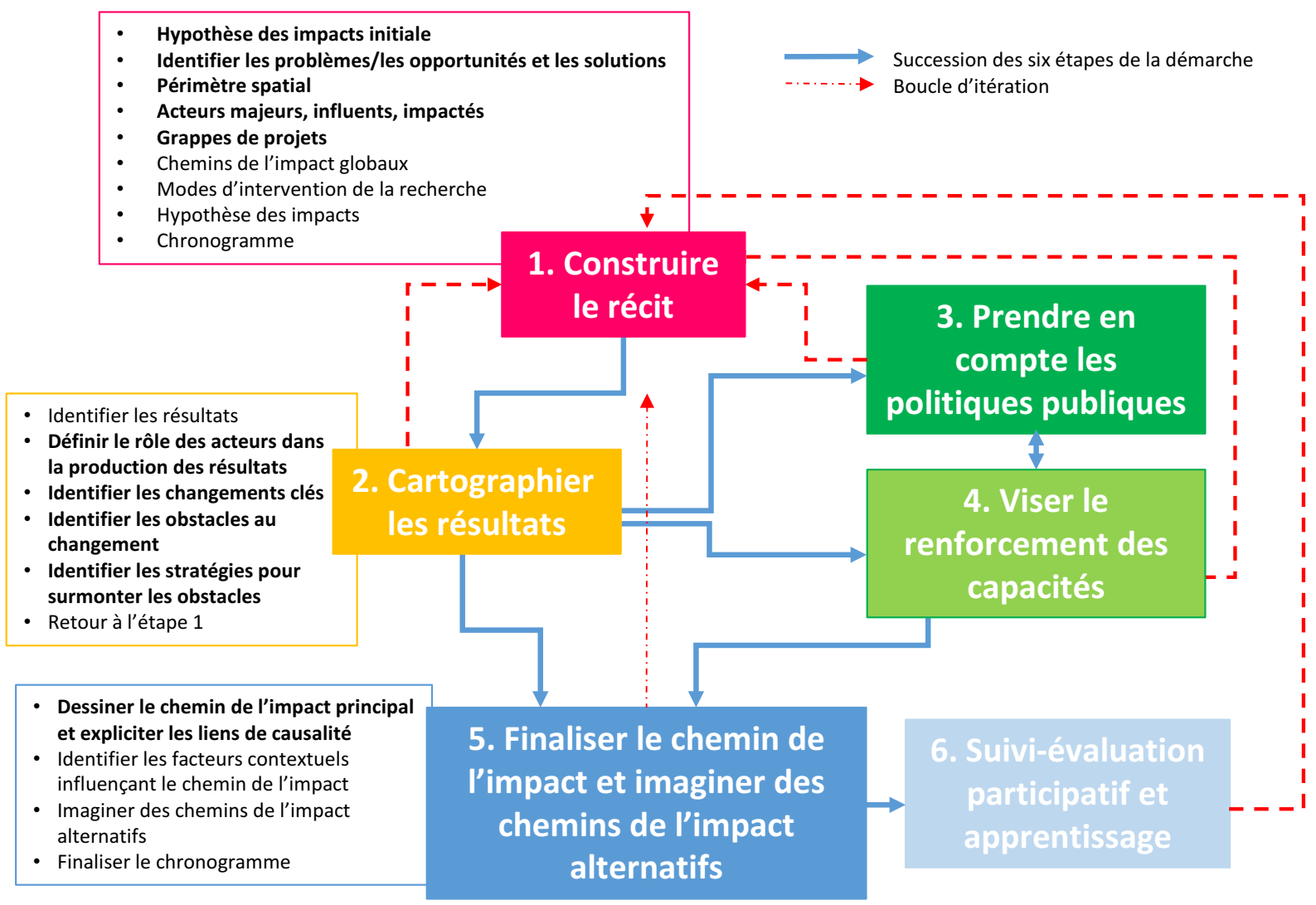

Figure 2 : La démarche ImpresS ex ante, un processus itératif en six étapes. 


\section{Une démarche adaptative, itérative et participative}

La démarche ImpresS n'est pas normative, ce n'est pas une recette à appliquer ; elle se veut flexible et adaptable à différentes exigences de temporalité et de ressources. Elle peut être déployée sur certaines étapes seulement, avec une intensité qui dépendra des moyens humains et matériels disponibles, ainsi que des délais impartis (voir partie "Adapter la démarche ImpresS ex ante à différents besoins "). Tout en s'appuyant sur un processus unique, la façon de présenter les résultats de la réflexion et le niveau de détail fourni, notamment pour la restitution du récit, dépendent de l'audience ciblée (producteurs, bailleurs, chercheurs, acteurs publics, ONG, etc.).

La démarche ImpresS ex ante vise à équiper à la fois des projets pris isolément, des programmes et des " grappes de projets", mais le niveau de spécificité et de détail varie avec le " grain " de l'intervention. L'identification des acteurs, des produits et des résultats sera plus ou moins fine selon que l'on construit une intervention qui est une grappe de projets ou qui se déroule sur plusieurs sites, ou une intervention qui est construite dans un périmètre circonscrit localement. Lorsque l'intervention est une grappe de projets, on formulera un chemin de l'impact générique valable pour le niveau le plus englobant, sur le temps long, tandis que des chemins de l'impact spécifiques seront développés à des niveaux plus locaux (au niveau de chaque projet de la grappe, au niveau de chaque site d'un même projet, etc.]. Ces chemins de l'impact spécifiques seront emboîtés de façon cohérente dans le chemin de l'impact global, qui représentera l'orientation et la vision commune de la grappe de projets ou du programme. Le chemin de l'impact global mettra en scène des types plus génériques d'acteurs, tandis que les chemins de l'impact spécifiques travailleront avec des acteurs réellement impliqués dans le projet, pour permettre de décrire précisément les actions à mener et les indicateurs de suivi à considérer.

Cette démarche est donc adaptative. Les équipes s'engageant dans la démarche ex ante ne sont pas obligées de la déployer dans sa totalité et peuvent n'approfondir que ce qui leur semble le plus pertinent pour leurs besoins propres. Par contre, le déroulement de la démarche dans sa totalité peut les aider à définir un récit plus robuste, des chemins de l'impact plus plausibles et une planification plus stratégique.

La démarche consiste en un processus itératif. Chaque étape de la démarche peut fournir des produits ou des rétroactions susceptibles d'améliorer les étapes précédentes (voir figure 2). Cependant, l'intensité effective du processus itératif dépend de la nature de l'intervention et des ressources (en temps, en capital financier et humain] que les chercheurs et leurs partenaires peuvent mobiliser.

Idéalement, la réflexion ex ante se mène dans le cadre d'un processus participatif impliquant les partenaires et les acteurs majeurs de l'intervention, ce qui permet de construire une vision partagée et d'augmenter la plausibilité du chemin de l'impact envisagé. Toutefois, la démarche peut être mise en œuvre même si les ressources permettant d'impliquer les partenaires avant de soumettre une proposition à financement manquent. Dans ce cas, il sera souhaitable, voire indispensable, d'inclure dans la proposition une demande de financement spécifique pour organiser un atelier participatif en tout début d'intervention dans l'objectif d'améliorer et de valider le chemin de l'impact ex ante avec les partenaires et/ou les acteurs majeurs.

Dans une évaluation ex ante, on se situe dans le domaine des hypothèses, avant la mise en œuvre de l'intervention (encadré 2). II s'agira donc d'argumenter sur la plausibilité de ces hypothèses, c'est-à-dire sur la capacité effective de l'intervention proposée de générer les impacts souhaités. L'argumentaire sera nourri par un travail rigoureux sur les aspects suivants : définition du périmètre de l'innovation et notamment des grappes de projets inscrites dans des trajectoires d'innovation, identification des hypothèses des impacts, des acteurs et des interactions qui favorisent l'innovation, construction des chemins de l'impact incluant l'analyse des risques et facteurs de réussite et enfin réflexion sur les scénarios alternatifs.

\section{Encadré 2 : ex ante, in itinere, ex post}

L'évaluation ex ante est menée pendant la phase précédant la mise en œuvre d'une intervention, c'està-dire dans la phase de conception. L'évaluation in itinere renvoie à une évaluation menée chemin faisant, pendant la mise en œuvre de l'action ou de l'intervention, tandis que l'évaluation ex post se fait une fois achevée l'intervention - projet, grappe de projets ou programme - dont on veut mesurer l'impact. 



\section{Les six étapes de la démarche ImpresS ex ante}

\section{Étape 1. Construire le récit}

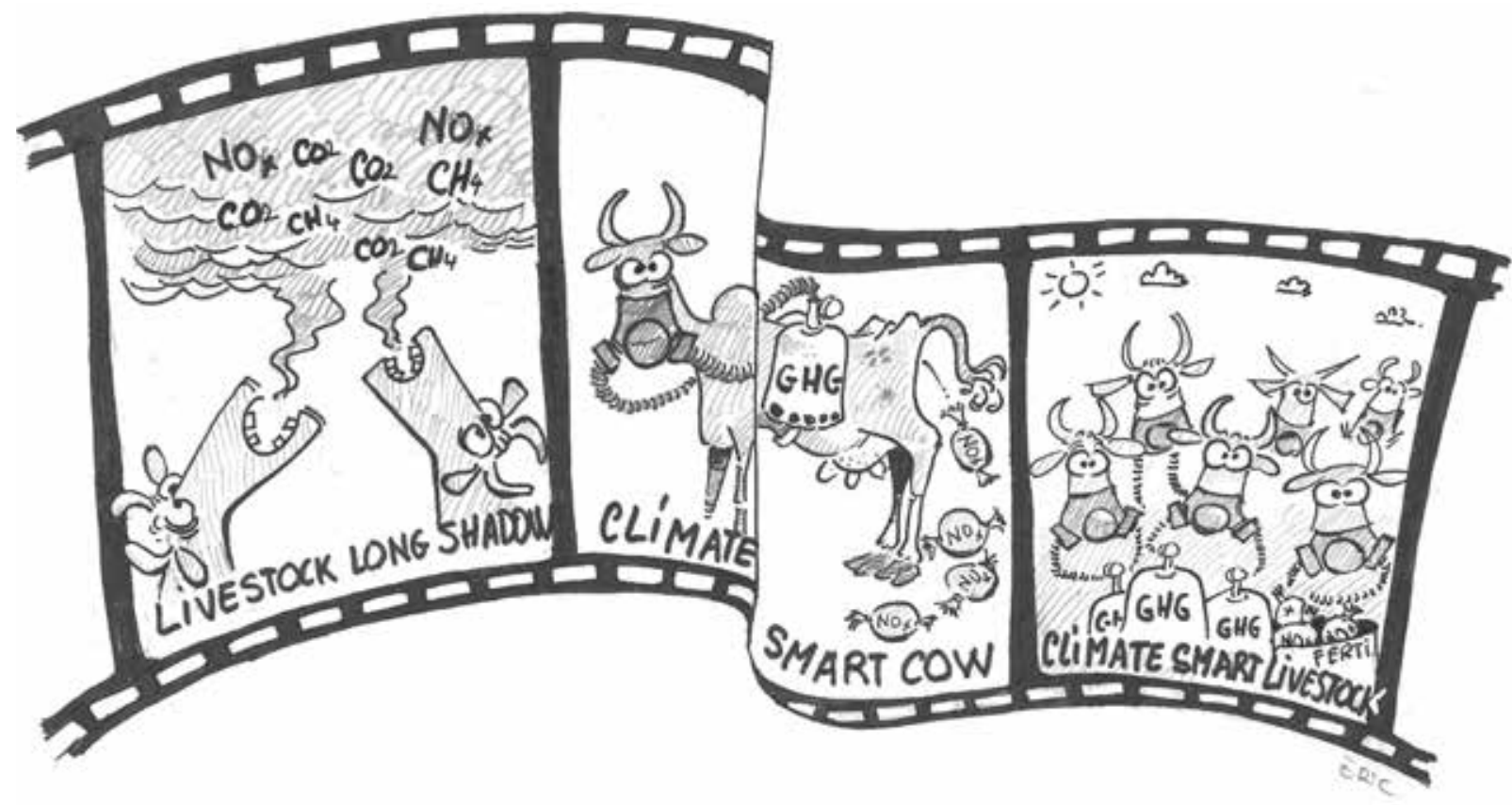

La démarche ImpresS ex ante vise à construire un récit de l'impact convaincant ${ }^{7}$ et plausible pour favoriser la création d'une vision partagée et la mobilisation des acteurs vers un objectif commun. Ce récit puise dans les interventions qui ont précédé et porté sur une problématique proche, la connaissance qu'en ont les acteurs, de même que les recherches antérieures. Il ne se résume pas à une juxtaposition d'arguments ou d'étapes à franchir, mais résulte d'un véritable exercice rhétorique de mise en forme des actions que l'intervention propose d'implémenter et des objectifs qu'elle souhaite atteindre, pour différentes audiences (acteurs divers, bailleurs), en restant toujours dans la plausibilité et la rigueur. La démarche ex ante se construit à partir d'un récit répondant aux questions : "Quelle intervention ? Où et dans quels buts ? Pour qui et avec qui ? Comment attendre ces buts ? " Ces divers éléments servent à élaborer un récit plausible et un chemin de l'impact cohérent basé sur les résultats [outcomes].

Construire des récits et des chemins de l'impact ex ante est utile pour améliorer les pratiques de montage de projets, la mise en œuvre et le suivi de ces derniers, construire une vision commune et des partenariats solides. Cela sert aussi à montrer clairement les liens entre les produits d'une intervention et les changements liés au développement auxquels l'équipe aspire. Le produit final est un récit simple, court et convaincant, qui présente la finalité visée par l'intervention et les modalités d'action, et prend en compte le rôle, les intérêts et l'influence des différents acteurs.

7 Le mot « convaincant " est faible. Les anglo-saxons parlent d'un récit « compelling " c'est-à-dire qui s'impose, qui déclenche l'empathie. 
La construction du récit à partir des neuf ingrédients détaillés ci-dessous est proposée. Il ne s'agit pas simplement d'aligner ces ingrédients mais de les relier de façon cohérente dans un exercice littéraire, une mise en forme qui emporte l'adhésion.

\subsection{Quel est le diagnostic / constat initial ?}

Pour commencer, il faut recueillir et analyser les informations sur une situation, une problématique ou un contexte (enjeux des acteurs, sociaux, politiques, etc.) qui justifient l'intervention pour des améliorations concrètes et réaliser un diagnostic rapide.

Cette première étape peut être complètement ouverte quand les intervenants n'ont pas de connaissances préalables sur la situation ; elle peut être aussi limitée à une problématique bien connue sur la base d'une analyse ou d'un diagnostic préliminaires, d'informations existantes ou de consultations initiales provenant des intéressés. C'est aussi l'occasion d'identifier les contraintes et les ressources disponibles et de se renseigner sur les projets qui ont déjà été réalisés par d'autres acteurs, sur la même thématique.

\section{Trucs $\&$ astuces $n^{\circ} 1$ : Construction du diagnostic initia}

La démarche projet des chargés de valorisation du Cirad offre une liste des items à analyser lors du diagnostic initial, dont sont reprises les suggestions suivantes:

- contextes [Cirad, géographique, social, politique, etc.] ; - acteurs (qui agit pour transformer la situation ?] ;

- acteurs cibles [à qui sont destinées les activités qui seront mises en place par l'intervention ?] ;

- opportunités de financement ;

- projets existants.
La démarche projet offre aussi des suggestions concernant des outils d'analyse :

- la démarche d'étude avec les différents outils de recueil de données (la recherche documentaire, le questionnaire, l'entretien, l'observation] ;

- le remue-méninge, le QQOQCCP [Quoi, Qui, Où, Quand, Comment, Combien, Pourquoi] ;

- le diagramme causes-effets ou diagramme d'Ishikawa, le schéma d'analyse de champ de force.

\subsection{Quelle est l'hypothèse initiale des impacts / la vision du futur ?}

Le diagnostic / constat initial devra être relié à une première hypothèse des impacts. Cette espérance d'impact se base sur la nature de l'intervention que l'on veut mener et sur la façon dont elle pourrait contribuer à générer des impacts. Il s'agit d'expliciter une vision du futur à 10-15 ans et donc du changement pour lequel l'intervention souhaite créer des conditions favorables ou franchir quelques étapes.

Cette première hypothèse des impacts, cette vision du futur, peut être orientée par les besoins ou objectifs exprimés par des acteurs locaux (attentes locales] ou partenaires [attentes des partenaires], par les impacts attendus inclus dans un appel d'offre auquel on pense répondre (attentes des bailleurs], ou par des attentes sociétales telles qu'elles sont formulées dans des documents d'orientation politique (attentes sociétales]. Quelques exemples sont présentés dans l'encadré 3. II est bon de garder en tête pour le récit l'origine de cette hypothèse des impacts.

\section{Encadré 3 : Quelques exemples d'hypothèses des impacts tirés d'expériences d'analyses ex ante}

"In 2025, as a result of the systematic and widespread application of improved pest management practices, fruit losses due to fruit fly and anthracnose in Kenya and Senegal will decrease dramatically. This will lead to increased fruit production and quality, increased income for smallholders, improvement of food and nutrition security, and better health. The subsequent reduction of pesticide use should benefit to the environment, including the restoration of biodiversity and its ecological functions such as natural pest control" [Projet BIOPHORA].
" L'exploitation durable et légale des populations d'animaux sauvages par les acteurs du monde rural dans les paysages clés pour la conservation de la faune, ainsi que la diversification de l'offre en protéines alternatives provenant d'animaux domestiques, assurent une nourriture carnée suffisante et de qualité pour assurer la sécurité alimentaire et nutritionnelle des populations, réconciliant sécurité alimentaire et conservation de la faune dans les pays d'Afrique, Caraïbes et Pacifique » (Projet Sustainable Wildlife Management). 
Une façon relativement simple d'aborder la vision du futur est de se positionner par rapport à la trajectoire passée et présente (Altamirano, 2015). Dans le cas d'une intervention qui porterait sur le territoire, le groupe choisit des thématiques d'intérêt, par exemple les sols, l'eau, la biodiversité, le genre, les familles, etc. Pour chaque thématique, il réalise une description des acteurs du territoire dans quatre situations :

1. Description du territoire, des acteurs et de leurs interactions il y a 30 ans ;
2. Description du territoire, des acteurs et de leurs interactions maintenant ;

3. Description du territoire, des acteurs et de leurs interactions d'ici 15 ans si la trajectoire actuelle n'est pas modifiée;

4. Description du territoire, des acteurs et de leurs interactions d'ici 15 ans si l'intervention travaille vers le changement.

À la fin, le groupe écrit une phrase ou un paragraphe qui synthétise la vision du futur.

\subsection{Quelle est la problématique centrale ? Quelle est l'opportunité ? Quelles sont les solutions?}

Formuler l'hypothèse initiale des impacts a permis d'expliciter une attente des acteurs. La réflexion est approfondie en identifiant les problèmes pour lesquels l'intervention doit apporter des solutions. L'analyse par un « arbre à problèmes » et un « arbre à solutions » (Chevalier et Buckles, 2008, p. 121) peut aider à défınir les problèmes, leurs raisons d'être et leurs conséquences (les branches plus « basses » et plus « hautes » de l'arbre permettent de hiérarchiser les problèmes), ainsi que les solutions (produits / outputs) potentielles (figure 3). Ces solutions sont une première ébauche de réflexion car c'est à travers un processus itératif et surtout grâce aux résultats des étapes suivantes - 2, 3, et 4 - que les solutions (produits / outputs) que l'intervention souhaite faire émerger et les conditions de leur émergence sont précisément définies.

a)

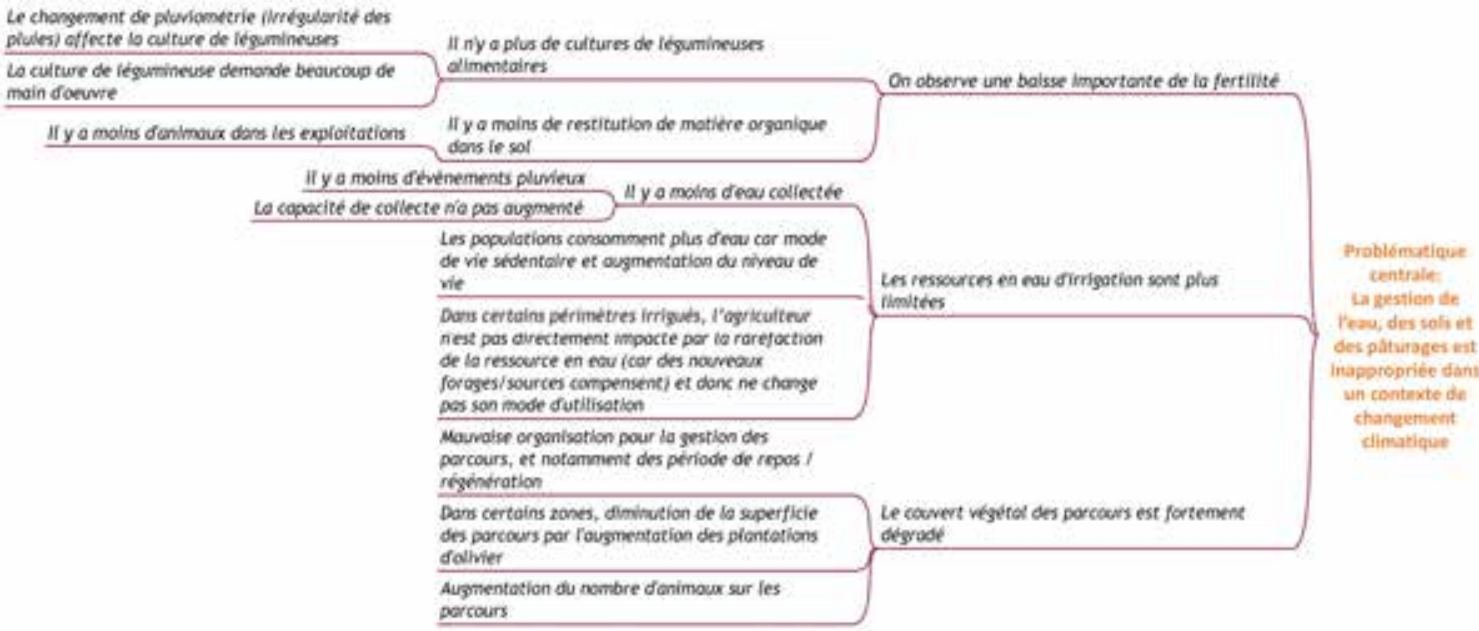

b) L'écosystème et les systèmes de production oasiens sont en équilibre face aux impacts du changement climatique

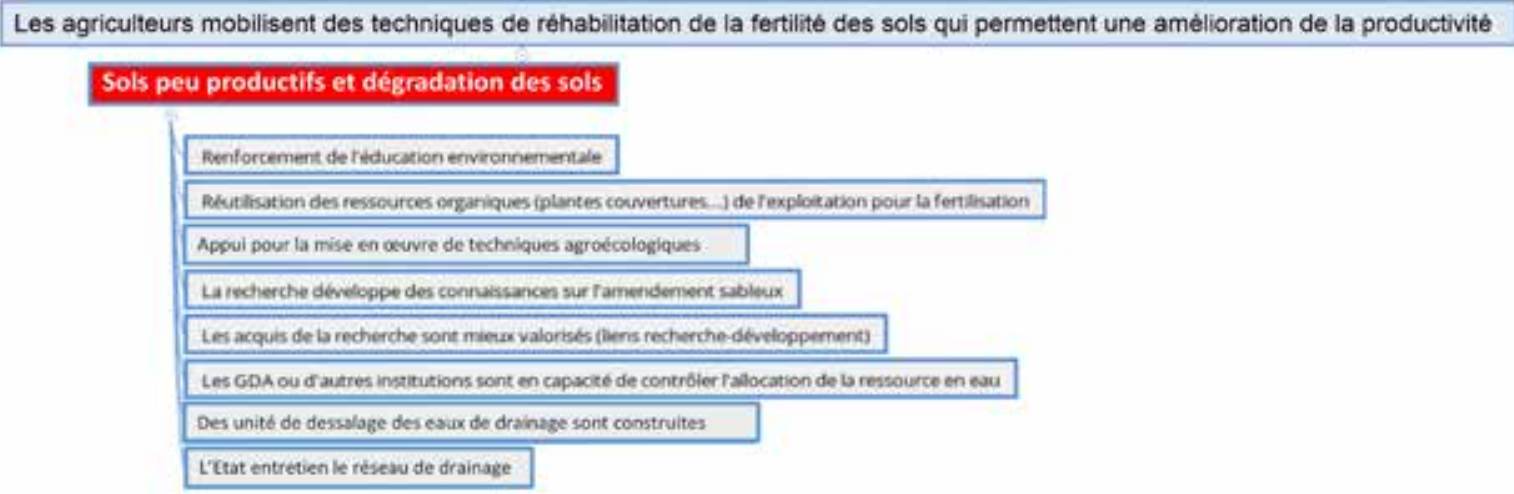

Figure 3 : Exemples d'arbre à problèmes [a] et d'arbre à solutions [b] [cas du projet GITES]. 
Dans les pratiques des chercheurs et des chargés de valorisation, l'arbre à problèmes et solutions est un outil souvent mobilisé. II existe plusieurs guides pour sa mise en œuvre et sa facilitation. Il est possible de capturer un arbre à problèmes ou à solutions sur Powerpoint ou via un autre logiciel, ou d'utiliser l'outil en ligne ImpresS [voir partie " L'interface ImpresS »).

Le programme CCAFS propose la méthodologie de facilitation suivante [Jost et al., 2014, p. 48] :

1. Demander aux participants quels sont 2-3 problèmes principaux qui empêchent que la vision du futur se réalise. Écrire ces problèmes dans la partie plus à droite d'un flip chart, un problème par feuille ;

2. Commencer avec le premier problème et demander pourquoi il existe. Il est utile de penser en termes de causes sociales, économiques, environnementales. II arrive de trouver une seule cause majeure ou d'en trouver plusieurs mineures. Les écrire à gauche du problème et les connecter avec une flèche au problème ;

3. Regarder les causes identifiées et se demander pourquoi elles existent. Écrire ces deuxièmes types des causes à gauche et les connecter aux premières causes identifiées.

4. Remarquer que vous êtes en train de dessiner un arbre avec des rames vers la gauche. II faut continuer à dessiner les autres rames en se demandant " pourquoi ». Cet exercice s'appelle " 5 pourquoi », parce que normalement il faut se demander " pourquoi " 5 fois pour arriver aux causes déterminantes et centrales [les racines]. Ce sont l'ensemble des causes qu'il faut aborder pour progresser vers la vision du futur, et elles aident à définir les produits [outputs] que l'intervention devrait générer pour contribuer au changement souhaité ;

5. Une fois tous les ramifications complétées jusqu'aux racines, vous pouvez arrêter. Après révision de l'arbre, vous pouvez identifier les causes que l'intervention peut travailler en rouge et celles que l'intervention ne peut pas travailler en noir ;

6. Faire le même exercice pour les autres problèmes.

Les chargés de valorisation du Cirad proposent également des orientations pour la construction d'un arbre à problèmes et solutions. L'exemple suivant est tiré du guide de facilitation de l'atelier de conception du projet « Adaptation au changement climatique en Tunisie » :

1. Votre idée d'intervention vise à répondre à un problème posé [constat initial / hypothèse des impacts]. Afın d'identifier des solutions adaptées, vous devez d'abord analyser ce problème, c'est-à-dire réfléchir à ses causes et conséquences : POURQUOI : Quelles sont les raisons / causes de cette situation ? ET ALORS : Qu'est-ce que cette situation entraîne comme conséquences là court / moyen / long terme] ? Essayez de réfléchir sans idée préconçue sur l'intervention :

- Mentionnez une cause / conséquence / idée par fiche bristol ;

- Chaque énoncé / idée doit être exprimé(e) comme une situation négative (manque de..., absence de...) ;

- Commencez par identifier toutes les causes / conséquences puis les relier entre elles par petits groupes avant de procéder à la construction de l'arbre.

2. Vous avez construit un arbre à problèmes à partir de votre diagnostic / constat initial... Mais vous ne pourrez pas tout résoudre avec une seule intervention.

- Sur l'arbre à problèmes, quels sont les points sur lesquels l'intervention peut apporter une amélioration?

- Si plusieurs chemins sont possibles, lesquels allez-vous choisir et pourquoi ? Préciser comment les voies choisies pour améliorer la situation initiale défınissent l'ambition et le périmètre du projet.

3. À ce point-là, vous pouvez passer à l'arbre à solutions. Vous allez tout d'abord formuler l'objectif précis que vous souhaitez atteindre grâce à l'intervention à partir du problème central que vous souhaitez résoudre. Quel est l'objectif concret que vous pensez pouvoir atteindre grâce à l'intervention ?

- Utilisez un verbe actif, à l'infınitif [augmenter, améliorer, connaître, résoudre, etc.] ;

- Formuler les objectifs plus généraux auxquels contribuera votre intervention ;

- Quelles sont les conséquences à long terme que l'intervention va améliorer ? Utilisez l'expression " contribuer à ... ".

\subsection{Quelles autres initiatives, quels autres projets passés, présents, futurs sont intervenus ou vont intervenir sur la même trajectoire d'innovation ? Comment prendre en compte une grappe de projets?}

Le temps de génération des impacts est long $[10,20$ ans ou plus $)$ et un projet, généralement d'une durée de 3 à 5 ans, ne peut prétendre obtenir à lui seul un impact significatif. De fait, une trajectoire d'innovation [voir Clossaire) se construit sur un ensemble d'interventions passées et en cours, reliées entre elles, et contribuant par un effet cumulatif à produire un impact ; la méthode ImpresS se réfère à cet ensemble comme à une grappe de projets (aussi identifiable comme "l'écosystème " du projet). Le périmètre pertinent à prendre en compte dans la conception de toute nouvelle intervention inclut donc les projets passés, en cours et futurs contribuant à une trajectoire d'innovation. 
Concrètement, cela nécessite de réaliser un diagnostic en modes rétrospectif et prospectif à partir des différentes interventions qui ont traité la problématique que l'on se propose de travailler :

- En mode rétrospectif : la nouvelle intervention fait-elle partie d'une trajectoire d'innovation existante?

- Qui a travaillé sur le sujet (interventions mais aussi acteurs non organisés] ?

- Quelles solutions ont été mises en œuvre?

- Avec quels partenariats?

- Quels produits et/ou quels résultats ont été obtenus ?

- Quels apprentissages tirer de leur expérience pour construire le récit de l'impact ?

- En mode prospectif : la nouvelle intervention fait-elle partie d'une grappe de projets ou d'interventions qui vont travailler sur cette problématique ou ce même territoire, ou sur la même filière ?

- Y a-t-il d'autres projets financés ou d'autres initiatives prêtes à démarrer sur cette problématique?

- A-t-on besoin de se coordonner pour tirer parti d'apprentissages communs ou de synergie d'actions?

- Si ces autres projets ou initiatives ont des objectifs concurrents ou divergents avec la nouvelle intervention, comment réduire les risques?

L'exemple suivant illustre une analyse de grappe de projets. Dans l'atelier du projet GITES [" Gestion intégrée des territoires en zones semi-arides "), I'analyse de la grappe de projets consistait à demander aux partenaires de positionner leurs projets en cours d'exécution par rapport aux quatre piliers de GITES que sont la gouvernance, la prospective, le dispositif de partage d'information et la production de connaissances. L'objectif était d'avoir un aperçu des champs d'intervention et de recherche investis par les participants à l'atelier et de leurs intérêts par rapport à ces quatre piliers, ainsi que d'expliciter leur implication et positionnement dans GITES. La figure 4 présente une cartographie des grappes de projets dans lesquels les participants de l'atelier GITES étaient impliqués, pour montrer comment GITES se place en continuité des travaux en cours, ce qui facilite le positionnement des uns et des autres sur le travail à faire ensemble.

OBIECTF SPECIFIQUE : PROPOSER DES SCENARII DE DEVELOPPEMENT POUR UNE GESTION INTEGREE DES RESSOURCES DANS LA VALLE DU FLEUVE SENEGAL ET L'OFFICE DU NIGER

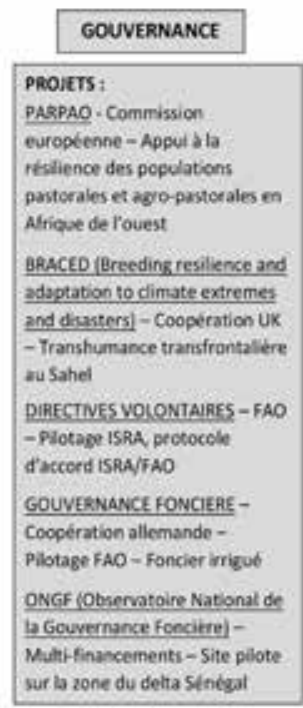

\begin{tabular}{|c|c|}
\hline PROSPECTIVES & PARTAGE DE L'TNFORMATION \\
\hline 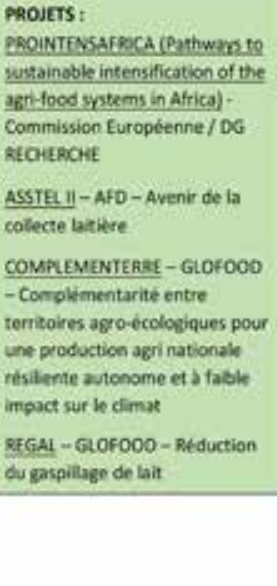 & 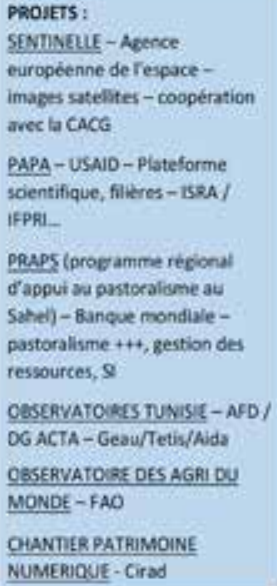 \\
\hline
\end{tabular}

\begin{tabular}{|c|c|}
\hline PROOUCTION DE C & NNAISSANCES \\
\hline 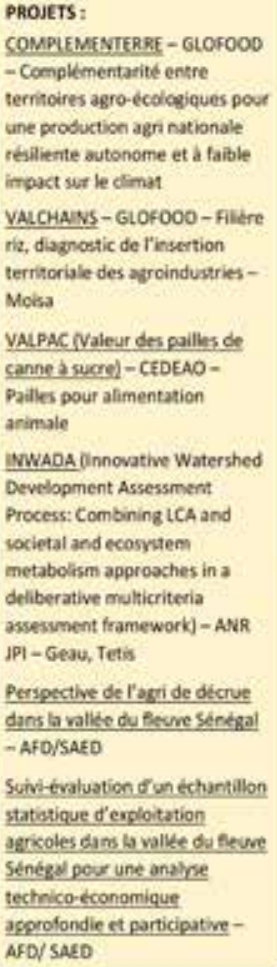 & 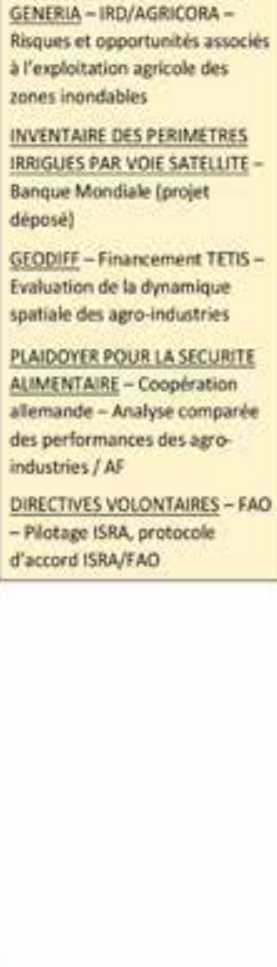 \\
\hline
\end{tabular}

Figure 4 : Une partie de la grappe des projets identifiée par le projet GITES. 


\subsection{Qui sont les acteurs majeurs, influents et impactés de l'intervention ?}

Il est ensuite nécessaire de mieux définir les acteurs impliqués dans le processus d'innovation (voir Glossaire) visé par l'intervention. La première " carte d'acteurs " obtenue n'est pas forcément complète à cette étape ; elle sera cependant d'autant plus précise que l'équipe projet aura participé à des interventions similaires dans la même région et/ou aura associé ces différents acteurs tôt dans l'élaboration de l'intervention. Quatre catégories d'acteurs sont utilisées pour la cartographie :

1.Les acteurs majeurs partenaires de l'intervention, avec lesquels l'équipe projet aura une interaction directe afin de livrer ensemble des produits 8 . Un acteur partenaire est un acteur qui apporte un investissement intellectuel, matériel, humain ou financier substantiel ; il participe formellement à l'intervention à travers une convention définissant les termes du partenariat entre les acteurs majeurs et parfois avec le bailleur ;

2.Les acteurs majeurs, qui sont clés dans le processus d'innovation mais qui ne sont pas partenaires formels de l'intervention;

3. Les acteurs influents, qui sont susceptibles d'influencer positivement ou négativement le processus d'innovation (y compris l'appropriation des produits et la génération des résultats) sans avoir un rôle d'acteurs actifs dans le processus d'innovation;

4. Les acteurs impactés, qui sont positivement ou négativement impactés par le processus d'innovation.

Si la délimitation entre ces quatre catégories s'avère floue, il sera nécessaire de faire un choix qui sera explicité. Les catégories ne sont pas mutuellement exclusives : les acteurs majeurs peuvent être aussi impactés, tout comme les acteurs influents. Elles peuvent évoluer au cours du temps.

L'encadré 4 montre des différences et similarités du lexique ImpresS avec le lexique de la démarche projet. Encadré 4 : Partenaires, prestataires de service, bénéficiaires ?

La démarche ImpresS fait le choix d'avoir un lexique bien défıni en termes d'acteurs, de résultats, d'impacts, etc. Ce lexique vise à une compréhension commune des différents concepts mobilisés, pour faciliter la communication. Néanmoins, des définitions et des concepts différents existent dans les procédures de montage de projet, la littérature, etc.

Un partenaire participe au projet car celui-ci entre dans sa stratégie d'action et son mandat en tant qu'organisation ou institution : une convention, définissant les termes du partenariat, est passée entre l'organisation porteuse du projet et l'organisation partenaire (ou indirectement entre l'organisme partenaire et le bailleur].

Un prestataire de service est un acteur qui effectue une tâche définie dans le cadre d'un contrat et pour laquelle il est rémunéré. II n'a pas d'influence sur le projet. Dans le lexique ImpresS, ce type d'acteurs, qui fait référence à la gestion ou à la mise en œuvre d'une intervention, n'est pas spécifiquement pris en compte.

Groupes cibles : ensemble d'individus à qui sont destinés les produits et résultats des activités qui seront mises en place par l'intervention. La démarche Impress distingue les acteurs majeurs partenaires de l'intervention, qui participent à la production des résultats [certains agriculteurs, par exemple, peuvent être des partenaires], et les acteurs impactés notamment dans les phases de changement d'échelle. Les groupes cibles peuvent faire référence aux deux publics.

Bénéficiaires finaux : individus qui verront leur situation modifiée indirectement par l'intervention (effet multiplicateur, changement d'échelle, etc.). Ils peuvent en outre valoriser les résultats de celle-ci pour poursuivre des objectifs qui leur sont propres. Dans le lexique ImpresS, ils correspondent aux acteurs impactés [incluant les acteurs impactés négativement).

Parties prenantes : individu ou collectif (groupe / organisation] concerné par une intervention, dont les intérêts peuvent être affectés positivement ou négativement à la suite de l'exécution [ou la non-exécution] de celle-ci. Dans le lexique ImpresS, ils correspondent aux acteurs impactés.

\subsection{Quelles interactions et relations existent entre les acteurs ?}

Cartographier les acteurs signifie aussi identifier leurs interactions (encadré 5). Ils peuvent être liés par exemple par des activités communes de recherche ou de développement, des flux de financement ou d'information, des intérêts communs ou encore des relations conflictuelles.

8 Par exemple, si dans une intervention seuls les chercheurs produisent les outputs, ceux-ci sont simplement des produits de recherche. Si d'autres types d'acteurs sont impliqués dans l'intervention, tels que des ONG ou des organisations de producteurs, alors les produits de l'intervention sont ceux du partenariat. 
Un réseau est un ensemble d'acteurs qui entretiennent des relations fortes ou faibles. La carte des acteurs est une visualisation des relations entre ces acteurs. Elle permet d'analyser quels acteurs / individus ont un rôle influent dans les réseaux (au centre de différents échanges, rôle d'intermédiaires, etc.), quels acteurs sont périphériques ou dépendants des autres. Elle se concentre sur les relations entre acteurs plutôt que sur leurs caractéristiques individuelles (Durland et Fredericks, 2005]. L'analyse de réseaux sociaux peut donner lieu à l'utilisation d'outils mathématiques pour analyser les relations entre les individus. Les données sont collectées à travers des enquêtes, des entretiens ou des focus groups. Cartographier le réseau d'acteurs est la première étape de l'analyse des réseaux sociaux.

Dans la démarche ImpresS, on cherche à comprendre les relations entre les différentes parties prenantes de l'innovation, ainsi que leur rôle dans le processus d'innovation. Une carte où les acteurs sont représentés par des nœuds et les relations entre les acteurs par des liens entre ces nœuds est ainsi élaborée. Les acteurs peuvent être des individus spécifiques agissant au sein d'une organisation, ou plus globalement des organisations. Les liens peuvent être représentés de manière simplifiée, sans en spécifier la nature ou l'intensité, mais il peut être utile de traduire ces liens en verbes d'action. Il est possible de représenter l'intensité des liens en faisant varier l'épaisseur de la flèche ou de représenter ces liens dans le temps pour montrer par exem ple comment ils se sont densifiés au cours du processus d'innovation.

Les liens peuvent représenter des flux d'échanges d'éléments de natures différentes. Il peut s'agir de flux d'information et de connaissance, de matériel ou financier. De plus, ces liens peuvent représenter différentes interactions de collaboration ou de rivalité avec des gradients possibles (coopération, compétition, compétition, conflit, etc.). Ces liens peuvent aussi correspondre à des relations de hiérarchie ou d'influence de certains acteurs vis-à-vis d'autres.

L'équipe ImpresS propose un outil numérique pour cartographier les acteurs d'une forme relativement simple et applicable hors connexion internet [voir partie "L'interface Impress »). L'interface ImpresS permet de visualiser les acteurs, de les identifier par couleur par rapport à leur rôle (majeur, majeur partenaire, influent, impacté] et de donner un nom au type de relation entre eux. C'est une visualisation simple qui appuie le travail de cartographie pendant un atelier avec les partenaires ou pour saisir et visualiser rapidement l'information.

Il est nécessaire de caractériser ces relations dans le cadre de la problématique centrale de l'intervention. Il est important d'identifier les acteurs qui ont une influence sur d'autres acteurs pour comprendre les enjeux à considérer et la structure institutionnelle dans laquelle l'intervention va s'insérer [voir figure 5].

Les liens entre les acteurs majeurs et les acteurs impactés d'une part et entre les acteurs influents et les acteurs impactés d'autre part sont particulièrement importants à prendre en compte pour réfléchir sur les changements d'échelle verticaux [scaling up] et horizontaux [scaling out].

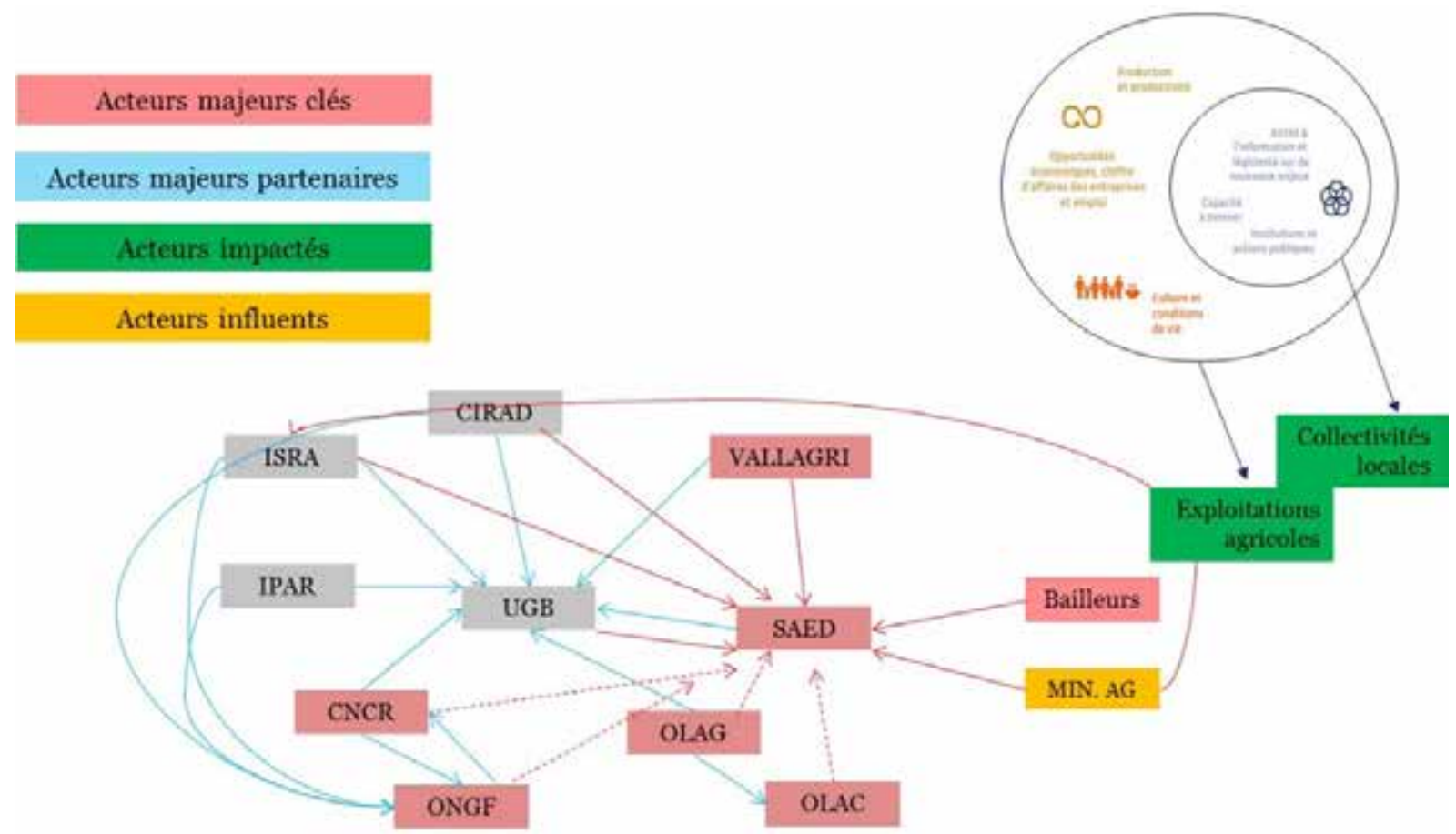

Figure 5 : Exemple de cartographie d'acteurs adaptée du projet GITES. 


\subsection{Quel est le périmètre sur lequel travailler ?}

Après cette évaluation des acteurs, le périmètre de l'intervention est identifié en détail. Il faudra définir à la fois une zone d'intervention et la zone plus large qu'on envisage d'impacter à travers des mécanismes de changement d'échelle. Ce périmètre peut représenter non seulement un espace géographique (territoire) et temporel (durée de l'intervention par exemple), mais aussi une filière ou tout un secteur agro-industriel. Les questions clés sont : quelle est la zone d'intervention cohérente avec les partenaires et ressources mobilisables ? Quel espace l'intervention envisage-t-elle d'impacter au-delà de la zone d'action, via des activités de changement d'échelle?

\subsection{Selon quels chemins de l'impact globaux travailler ?}

Il peut être intéressant de positionner l'intervention à la fois au regard (i) de trois chemins de l'impact globaux donnés par la littérature sur l'innovation et (ii) d'un modèle d'intervention de la recherche que l'équipe projet est en mesure de mettre en œuvre pour soutenir le processus d'innovation. Douthwaite et al. (2017) proposent un modèle dans lequel la recherche agricole pour le développement contribue aux impacts sociétaux et environnementaux selon trois chemins de l'impact interconnectés (Figure 6) :

1. L'impact via l'adoption des technologies par les acteurs - le chemin du développement et d'adoption technologique. Le chemin du développement et d'adoption technologique est familier à la plupart des chercheurs (Douthwaite et al., 2017) et renvoie au modèle de transfert linéaire de technologies. C'est une simplification de la réalité du développement technologique dans des trajectoires d'innovation existantes, comme la sélection variétale pour la résistance aux maladies, ou la mécanisation de l'agriculture ;

2. L'impact par le renforcement de la capacité à innover au sein des systèmes d'innovation agricoles, ou via l'initiative locale ou la conquête sociale - le chemin de développement endogène : des processus de recherche collaborative et participative construisent la capacité à innover des acteurs ruraux et des organisations d'accompagnement des acteurs ruraux. Ce chemin se base sur la nécessité d'améliorer la capacité à innover et les interactions des acteurs qui contribuent à des objectifs de développement communs. Les approches participatives et collaboratives sont essentielles et identifient des défis communs en construisant un capital social cognitif structurel dans le processus, favorisant un développement endogène ;

3. L'impact à travers l'influence de la sphère politique - le chemin de l'influence politique : la recherche génère des idées, établit des faits avec l'intention d'influencer la décision politique. Le changement politique aide la construction d'un environnement favorable pour l'innovation rurale.

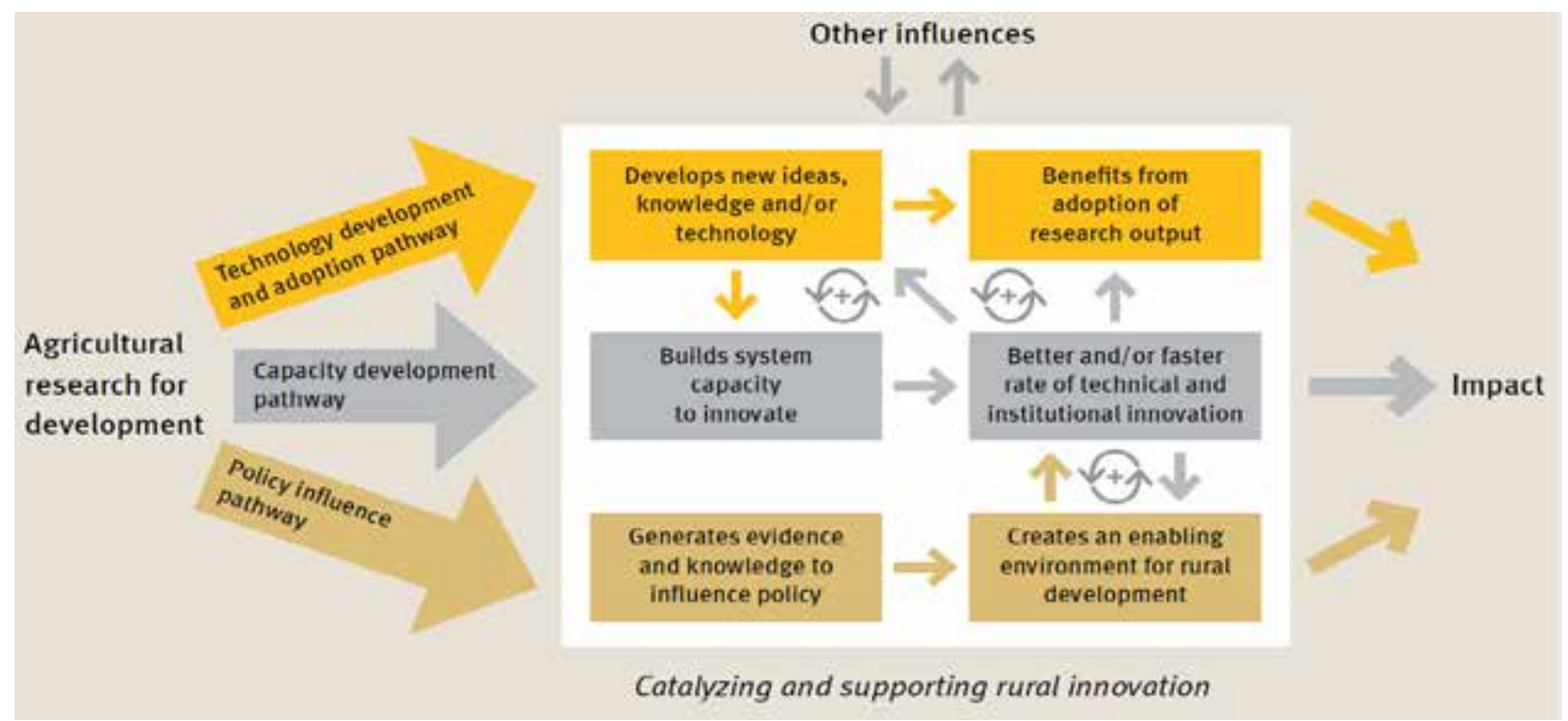

Figure 6 : Trois chemins de l'impact globaux interconnectés qui montrent la contribution de la recherche à l'impact [Douthwaite et al., 2017, p. 5].

Selon Douthwaite et al. (2017), toute intervention de recherche agronomique pour le développement aurait un impact résultant d'une combinaison de ces trois chemins. De fait, des interactions et des boucles de rétroaction 
positives entre ces chemins constituent souvent les éléments déclencheurs pour générer des résultats : par exemple, une action de recherche sur des dimensions techniques impliquerait aussi de renforcer les capacités à innover des acteurs pour favoriser l'appropriation de la technologie prévue, et ensuite exigerait des interactions avec la sphère politique pour le changement d'échelle.

Dans le cadre du montage d'une intervention, les trois chemins de l'impact peuvent être interconnectés dans le temps, et la recherche peut contribuer à l'impact selon différents chemins à différents moments.

Identifier comment l'intervention se positionne par rapport aux trois types de chemins possibles peut aider à orienter les étapes suivantes en termes d'activités, d'acteurs à engager et de types de résultats à viser. Par rapport au récit, cela aide à expliquer directement et simplement sur quel type de chemin l'intervention est positionnée (adoption technologique, développement endogène par renforcement des capacités ou influence politique).

\subsection{Quel est le mode d'intervention principal de la recherche?}

Pour orienter la réflexion sur les activités à mener, les acteurs à engager et les résultats à viser, il peut être utile d'expliciter le mode opératoire de la recherche dans l'intervention. La façon dont la recherche interagit avec les autres acteurs de l'innovation est un des éléments qui configure le chemin de l'impact. Trois éléments interviennent : (i) le poids des connaissances scientifiques dans le processus d'innovation et le niveau de contrôle que la recherche exerce sur les technologies qui en sont issues, (ii) le rôle que la recherche se voit jouer pour piloter le chemin de l'impact et (iii) le poids des autres acteurs dans le processus d'innovation et d'orientation vers les impacts. En fonction de ces éléments, et selon son mode d'intervention, la posture de la recherche et son contrôle sur les produits, les résultats et de l'ensemble du processus varieront (Devaux-Spatarakis et al., 2016).

Les recherches menées au Cirad peuvent s'inscrire dans quatre " archétypes » qui ont été décrits lors de l'analyse de 13 études de cas dans le cadre de la démarche ImpresS ex post (figure 7) :

- Transfert participatif de connaissances et de technologies (contrôle fort) ;

- Co-construction des innovations (contrôle moyen) ;

- Accompagnement du processus d'innovation [contrôle faible] ;

- Innovation ouverte [contrôle faible).

\section{Transfert participatif de connaissances et de technologies}

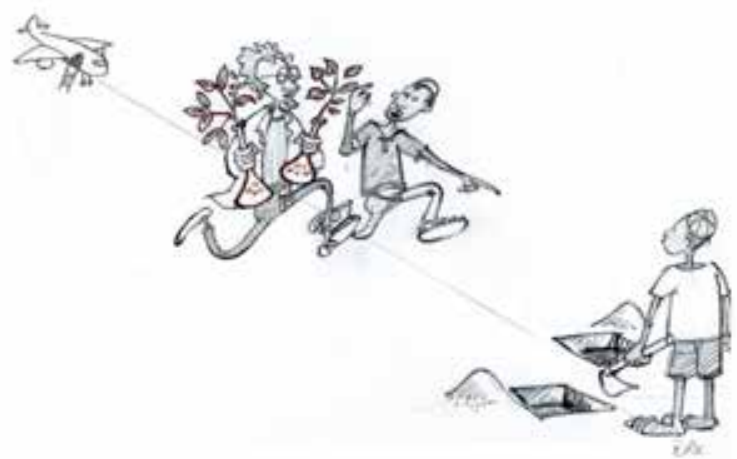

Accompagnement du processus d'innovation

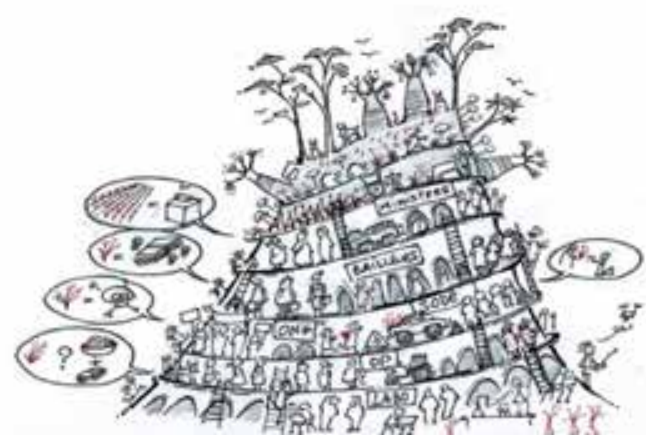

Co-construction des innovations

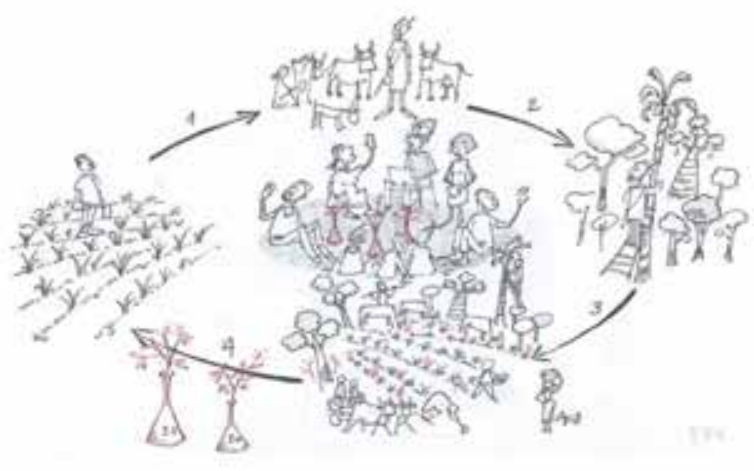

Innovation ouverte

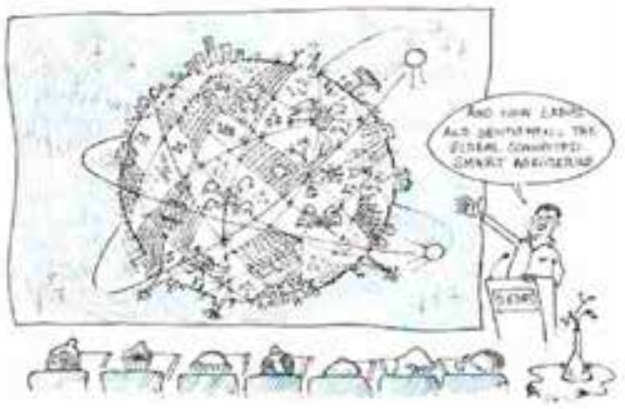

Figure 7 : Archétypes des situations d'intervention de la recherche au Cirad (Dessins : Éric Vall]. 
Dans le récit du chemin de l'impact, décrire le mode d'intervention de la recherche peut aider à mieux formuler la manière dont l'intervention va construire ses produits, par quels processus elle va s'efforcer d'atteindre ces résultats, et comment ces derniers vont catalyser des impacts. Cela peut aussi aider à expliciter comment la recherche va parvenir à atteindre ses objectifs alors que, dans certains cas, elle n'a pas le contrôle du chemin de l'impact.

\subsection{Boucle : affiner l'hypothèse des impacts}

Après avoir réfléchi aux différents points clés du récit, il est utile de revenir sur l'hypothèse des impacts et de commencer à l'affıner (tout en gardant à l'esprit que l'hypothèse des impacts ne sera finalisée qu'à l'issue de l'étape 5].

Les impacts suivants peuvent être distingués :

- Les impacts de $1^{\text {er }}$ niveau, concernant les acteurs interagissant directement ou indirectement avec la recherche ${ }^{9}$;

- Les impacts de $2^{\mathrm{e}}$ niveau, après un changement d'échelle (horizontal / scaling out, ou vertical / scaling up) de cette innovation à d'autres territoires ou d'autres publics, ou des spillovers [effets inattendus et non programmés de l'innovation). Ces impacts concernent des acteurs au-delà de ceux qui interagissent directement ou indirectement avec la recherche.

Lors de la réflexion concernant les impacts qu'il est souhaitable de générer, il ne faut pas se limiter aux impacts positifs ; il faudra également envisager les impacts négatifs, directs ou collatéraux, qui seront dans ce cas appelés risques (figure 8).

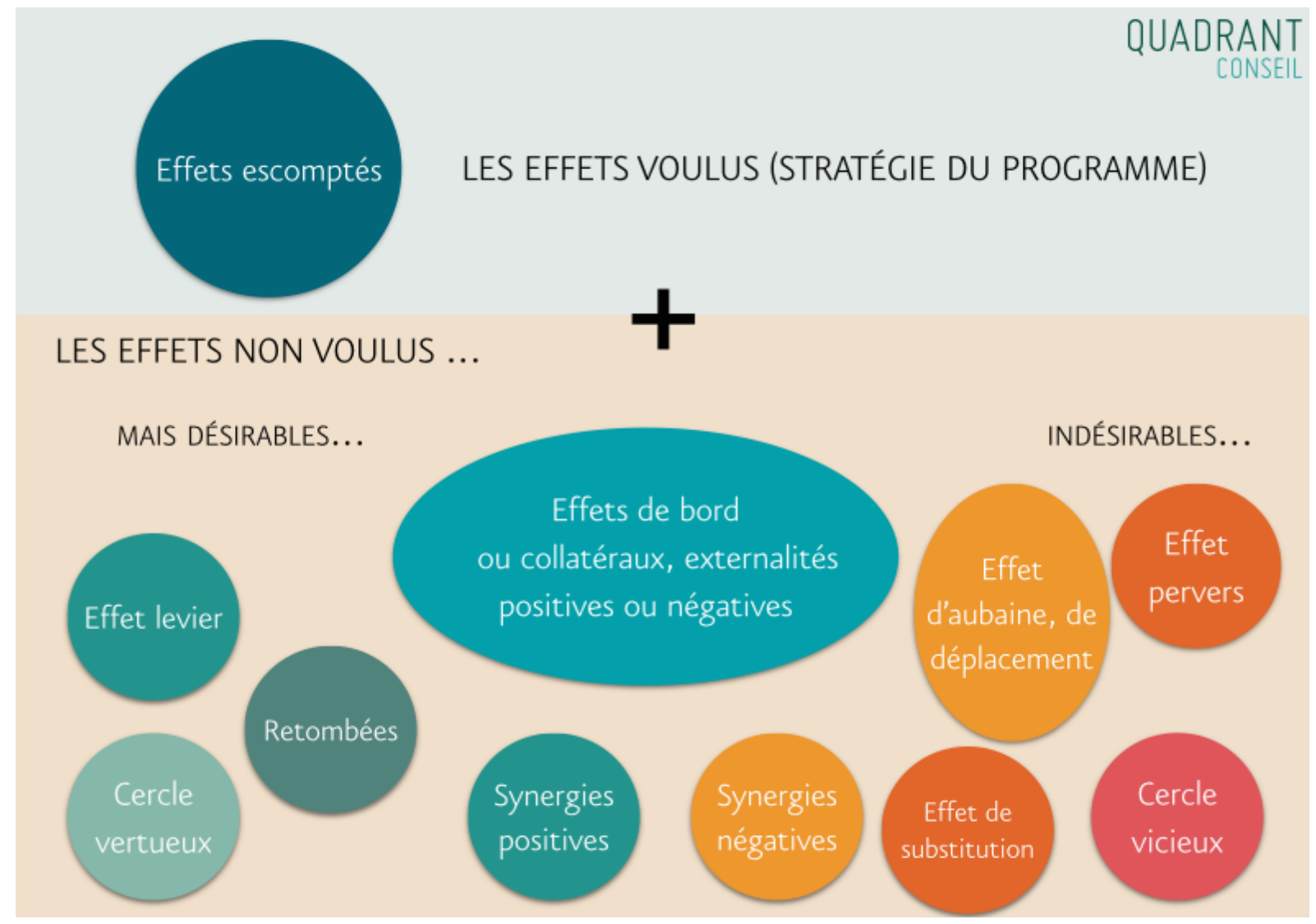

Figure 8 : Les différents types d'impacts.

9 Des impacts positifs de $1^{\text {er }}$ niveau issus d'une étude de cas réalisée dans le cadre de la démarche ImpresS ex post recouvrent une hausse des revenus des transformatrices de fonio, l'amélioration de la qualité du fonio décortiqué, etc. Les impacts positifs de $2^{\mathrm{e}}$ niveau concernent la hausse des revenus de producteurs de fonio dans la zone de production. 
Même si dans la démarche ex ante tous les effets possibles ne peuvent pas être prévus, la réflexion doit porter sur ceux qui seraient éventuellement impactés négativement par l'intervention et sur la façon de réduire ces risques. Cette réflexion sera complétée au cours de l'étape 2 pendant laquelle les résultats et les obstacles à l'appropriation des acteurs seront examinés.

Les équipes projet peuvent définir les impacts souhaités de leur intervention en référence aux onze domaines d'impacts identifiés par le Cirad sur la base des 13 études de cas analysées dans le cadre de la démarche ImpresS ex post, et qui peuvent être mis en correspondance avec les objectifs du développement durable [tableau 1, page suivante). La visualisation par le « radar des impacts » (figure 9) aide à communiquer de manière directe et claire sur les impacts visés par l'intervention. Ces radars constituent un support tant pour les interventions qui définissent les impacts sous un format quantitatif, que pour celles qui utilisent une définition qualitative. Dans la phase d'élaboration d'une intervention, il ne sera pas possible, sauf exception, de proposer une intensité dans les domaines d'impacts qui sont visés (longueur de la branche du radar].

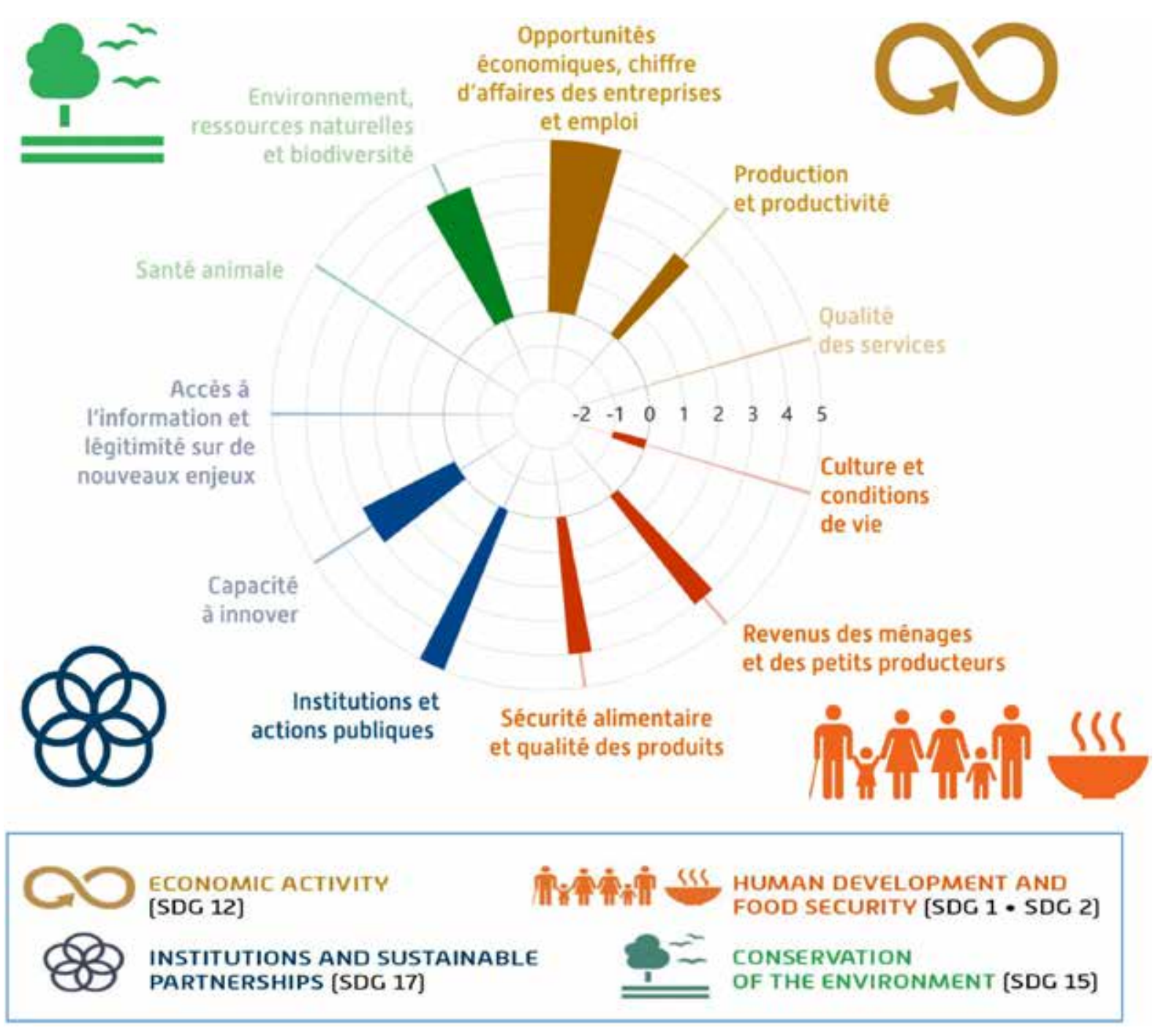

Figure 9 : Radar des impacts pour l'étude de cas « Sélection participative du sorgho au Burkina Faso ".

Le radar des impacts a été construit pour les études de cas ex post selon onze domaines qui couvrent les différentes missions du Cirad. Dans le cadre des études ex post, chaque domaine a été noté par scoring par un panel d'experts, en agrégeant les impacts qui s'y rattachent. La longueur des branches illustre l'intensité (de -5 à +5) et l'épaisseur figure l'ampleur (de 0 à 3) des impacts. 
Tableau 1 : Les 11 domaines d'impacts identifiés dans le cadre de la démarche ImpresS.

\begin{tabular}{|c|c|c|c|}
\hline Domaines d'impacts [11] & Dimensions d'impacts [4] & \multicolumn{2}{|l|}{ ODD concernés } \\
\hline Culture et conditions de vie & \multirow{3}{*}{$\begin{array}{l}\text { Développement humain } \\
\text { et sécurité alimentaire }\end{array}$} & $\begin{array}{l}\text { Éliminer l'extrême pauvreté } \\
\text { et la faim dans les milieux }\end{array}$ & ODD1 \\
\hline Sécurité alimentaire et qualité des produits & & Éliminer la faim, assurer la sécurité & \multirow{2}{*}{ ODD2 } \\
\hline Revenus des ménages et producteurs & & et promouvoir l'agriculture durable & \\
\hline $\begin{array}{l}\text { Environnement, ressources naturelles } \\
\text { et biodiversité }\end{array}$ & \multirow{2}{*}{$\begin{array}{l}\text { Préservation de } \\
\text { l'environnement }\end{array}$} & \multirow{2}{*}{$\begin{array}{c}\text { Préserver et restaurer } \\
\text { les écosystèmes terrestres, } \\
\text { les exploiter de façon durable, } \\
\text { mettre fin à l'appauvrissement } \\
\text { de la biodiversité }\end{array}$} & \multirow{2}{*}{ ODD15 } \\
\hline Santé animale & & & \\
\hline $\begin{array}{l}\text { Opportunités économiques, chiffre d'affaires } \\
\text { des entreprises et emploi }\end{array}$ & \multirow{3}{*}{ Activité économique } & \multirow{3}{*}{$\begin{array}{l}\text { Établir des modes de consommation } \\
\text { et de productions durables }\end{array}$} & \multirow{3}{*}{ ODD12 } \\
\hline Production et productivité & & & \\
\hline Qualité des services & & & \\
\hline Institutions et actions publiques & \multirow{3}{*}{$\begin{array}{l}\text { Institutions et partenariats } \\
\text { durables }\end{array}$} & \multirow{3}{*}{ Partenariats } & \multirow{3}{*}{ ODD17 } \\
\hline $\begin{array}{l}\text { Accès à l'information et légitimité } \\
\text { sur de nouveaux enjeux }\end{array}$ & & & \\
\hline Capacité à innover & & & \\
\hline
\end{tabular}

\subsection{Un chronogramme provisoire à compléter à la fin de la réflexion [étape 6]}

À ce stade, l'équipe projet est en mesure de spécifier provisoirement la chronologie d'ensemble de l'intervention et de son chemin de l'impact. Celle-ci reflète non seulement la chronologie de court terme du bailleur, centrée essentiellement sur les produits, mais aussi l'horizon auquel les produits, résultats et impacts pourraient se matérialiser. Qu'est-ce qui peut réellement être fait dans les temps impartis à l'intervention par l'agenda du bailleur? Est-il possible de montrer à travers les grappes de projets que l'intervention fait partie d'une trajectoire d'innovation qui va au-delà du temps du projet et que les impacts se matérialiseront de ce fait sur le long terme?

\subsection{Boucle : ajouter les résultats [outcomes]}

Le récit sera progressivement amélioré au fur et à mesure que la démarche ex ante sera déroulée et approfondie. Après avoir réfléchi aux résultats (voir partie " Étape 2 : Cartographier les résultats loutcomes] "), incluant le renforcement des capacités et l'interaction avec les acteurs publics [voir parties " Étape 3 : Prendre en compte les acteurs publics » et "Étape 4 : Viser le renforcement des capacités »), il faut revenir au récit et $y$ ajouter les résultats visés. Dans la structure du récit, les résultats sont placés juste avant des impacts souhaités (détaillés à la fin de l'étape 5) pour pouvoir expliciter le lien entre eux.

\subsection{Boucle : améliorer le récit avec le chemin de l'impact finalisé}

Après avoir finalisé l'étape 5 (voir partie « Étape 5 : Finaliser le chemin de l'impact et imaginer des chemins de l'impact alternatifs "], le récit sera amélioré : le chemin de l'impact représente en fait une visualisation synthétique du récit. Le récit doit donner une explicitation convaincante du chemin de l'impact et des mécanismes sous-jacents à la génération des résultats et d'impacts. 


\section{Ingrédient $\mathbf{n}^{\circ} \mathbf{1}$ : Orientation}

L'attention du lecteur doit être captée dès le début du récit. D'entrée, l'histoire doit l'orienter sur le contexte, l'introduire aux protagonistes. Le lecteur doit se sentir engagé dans ce que vivent les protagonistes.

\section{Ingrédient $\mathbf{n}^{\circ} \mathbf{2}$ : Crise / problème}

La crise ou le problème auquel doivent faire face les protagonistes n'est pas immédiatement soluble par eux. Le défi que représente cette crise justifie l'histoire.

Ingrēdient $\mathrm{n}^{\circ} \mathbf{3}$ : Découverte / innovation

Au climax de l'histoire, les protagonistes font une découverte / innovation qui change leur vie (l'intervention !). Typiquement, cette découverte / innovation résulte d'un choix des protagonistes. Ils peuvent être accompagnés par d'autres pour cette découverte / innovation (par exemple, les chercheurs !), mais la décision ultime revient aux protagonistes [l'appropriation par les acteurs !].

\section{Ingrédient $\mathbf{n}^{\circ} \mathbf{4}$ : Changement}

La solution de la crise / du problème est marquée par un changement et cela permet de conclure l'histoire : on montre que les actions, perceptions et attitudes des protagonistes ont changé par rapport au début de l'histoire [les résultats / outcomes !). Ces changements provoquent des effets sur leur vie quotidienne (les impacts !).

Inspiré de http://www.writersdigest.com/ online-editor/the-5-essential-story-ingredients 


\section{Étape 2. Cartographier les résultats [outcomes]}

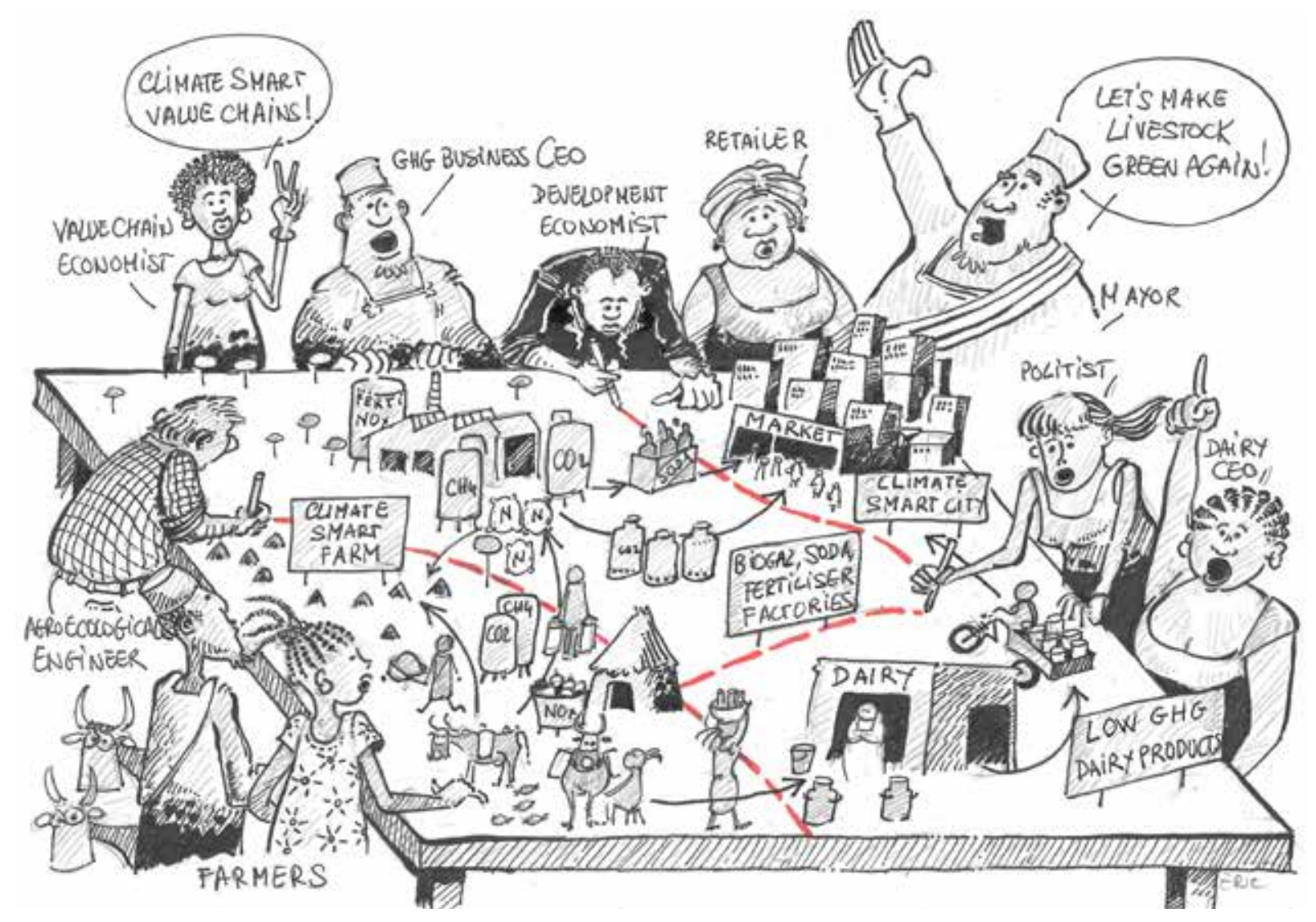

Une fois décrits les enjeux de l'intervention et les éléments tels que les impacts, les grappes de projets, les chemins de l'impact globaux et le mode d'intervention de la recherche, la réflexion doit se focaliser sur les acteurs et leur rôle de protagonistes des changements susceptibles de générer les impacts sociétaux et environnementaux souhaitables. L'intervention doit viser des changements plausibles et réalisables qu'elle est capable de catalyser en tenant compte des capacités et des ressources mobilisables dans le cadre de l'intervention.

Les changements nécessaires, mais hors de portée de l'intervention, nécessiteront d'identifier des stratégies alternatives pour les atteindre, par exemple via une collaboration avec d'autres projets. À la fin de cette deuxième étape, on sera mieux à même de définir les contours et les limites de l'intervention, ce qui constitue une boucle vers l'étape 1.

\subsection{Quels sont les résultats [outcomes] finaux souhaités? Qui fait quoi, différemment?}

Tout d'abord, il s'agit de se concentrer sur les acteurs et sur la manière dont l'intervention permet l'usage, l'adaptation, la transformation et au final l'appropriation de produits qu'elle aura générés, ou, en d'autres termes, comment l'intervention peut atteindre ses résultats.

La démarche ImpresS définit un " résultat " [outcome) comme l'appropriation d'un produit de la recherche par les acteurs interagissant directement ou indirectement avec l'intervention, qui conduit à des changements de pratiques (agricoles ou managériales), des changements organisationnels, d'interactions ou des règles. Cette appropriation représente des changements dans les pratiques ou les comportements de divers acteurs par rapport à la situation initiale. Ces changements permettent aux acteurs de générer un processus d'innovation, produisant in fine des impacts. Plusieurs exemples de résultats ont été identifiés dans les 13 études de cas analysées dans le cadre de la démarche ImpresS ex post. Ils sont repris 
ci-dessous, en explicitant le lien qu'ils entretiennent avec les produits d'une part, et les impacts d'autre part :

- Des agriculteurs qui adoptent ou adaptent (résultat / outcome) une nouvelle pratique agricole ou managériale [produit / output] constatent une réduction de leurs coûts de production et des pertes post-récolte, ce qui entraîne une augmentation de la production et des revenus (impact) ;

- Un projet qui expérimente un nouveau processus de transformation des produits et propose de nouvelles normes [produit / output] travaille avec des services de conseil agricole qui, avec d'autres acteurs, accompagnent la mise en œuvre de nouvelles technologies auprès des artisans ruraux (résultat / outcome), entraînant l'augmentation des revenus des ménages bénéficiant de leurs conseils (impact) ;

- Les acteurs qui font fonctionner un nouveau dispositif de formation (résultat / outcome), suite à la sensibilisation ou promotion du projet (produit / output), augmentent le recyclage de leurs déchets domestiques (impact) ;

- Les acteurs qui développent de nouvelles activités ou changent leurs pratiques managériales (résultat / outcome] sous l'impulsion d'une organisation de production, de commercialisation, ou de gestion territoriale élaborée avec l'aide de l'intervention (produit / output) contribuent à rendre plus équitable l'exploitation des biens communs (impact) ;

- Les acteurs qui changent leur manière de travailler leur terroir et de gérer leurs exploitations (résultat / outcome] en tirant partie des nouvelles organisations de coordination et/ou de suivi élaborées avec le projet (produit / output) contribuent à améliorer la gestion et à préserver la biodiversité (impact) ;

- Les acteurs qui renforcent leurs capacités et s'organisent mieux (résultat / outcome) grâce à des partenariats multi-acteurs et/ou de réseau impulsés par l'intervention (produit / output), contribuent à la résolution des conflits (impact) ;

- Un gouvernement qui, sur la base de travaux scientifiques liés à l'intervention (produit / output), établit une nouvelle norme (résultat / outcome), parvient à réduire l'empreinte environnementale des entreprises transformatrices (impact).

Cette présentation peut paraître très linéaire, mais c'est dans un but pédagogique, afın de faciliter l'identification des liens de causalité. Dans la réalité, cette relation est souvent nettement moins linéaire et plus complexe. Par ailleurs, la distinction entre produits, résultats et impacts n'est pas forcément tranchée : il s'agit en partie d'une convention de vocabulaire couramment utilisée dans le monde de l'évaluation et que la démarche ImpresS a retenue.

Afin d'être en cohérence avec la problématique / opportunité identifiée dans l'arbre à problèmes et solutions (voir partie "Étape 1: Quelle est la problématique centrale ? Quelle est l'opportunité ? Quelles sont les solutions ? "), il faut se demander à ce stade si les changements de pratiques et de comportements identifiés permettront effectivement de résoudre certaines des causes principales du problème motivant l'intervention.

\subsection{Quel est le rôle des acteurs dans la production des résultats [outcomes] ?}

Au niveau de l'étape de construction du récit, les acteurs impliqués dans le processus d'innovation visé par l'intervention ont été cartographiés et classifiés comme majeurs, majeurs et partenaires, influents et/ ou impactés.

Afın de comprendre au mieux comment produire les résultats souhaités, il faut répondre à plusieurs questions relatives aux rôles et à l'attitude des acteurs vis-à-vis des produits de l'intervention :

- Quel est précisément le rôle de chaque acteur dans la production des résultats (outcomes) ? Quelle est son influence probable?

- Comment la production des résultats (outcomes] affecte-t-elle chaque acteur?

- Quelle est la réaction prévisible de l'acteur à la solution proposée?

Les réponses à ces questions doivent être obtenues par type d'acteurs, notamment en distinguant les acteurs majeurs et les acteurs influents. Quand les réponses à ces questions ne sont pas disponibles pour l'équipe projet, une phase de diagnostic peut être envisagée lors du démarrage de l'intervention, afin de définir la meilleure stratégie à mettre en place pour engager les différents acteurs et favoriser l'appropriation des résultats ou modifier l'intervention et notamment ses produits. 


\subsection{Quels sont les changements majeurs souhaitables pour générer les résultats [outcomes] finaux et les principaux obstacles à ces changements ?}

À ce stade, il est nécessaire de se demander quels changements majeurs quant aux connaissances, capacités, attitudes et interactions / relations entre les acteurs sont nécessaires pour que les résultats se produisent, et dans certains cas, pour que les acteurs puissent participer à la génération des produits.

Il s'agit de se demander, par rapport à chaque résultat visé, quels changements sont nécessaires et souhaitables pour que les acteurs s'approprient le ou les produits de l'intervention.

Les changements majeurs provoqués par l'intervention représentent des résultats intermédiaires qui permettent d'arriver à un résultat final plausible. À nouveau, la distinction entre résultat intermédiaire et résultat final est une convention : certaines interventions considèreront comme des résultats finaux ce que d'autres considèrent comme des résultats intermédiaires. Cela n'est pas gênant, l'idée est plutôt de réfléchir aux stratégies et aux pratiques des acteurs et aux changements souhaitables pour changer l'état actuel des choses et déclencher des impacts.

Quelques questions peuvent être posées pour appuyer la réflexion :

- En relation avec la problématique centrale, quels acteurs devraient faire les choses différemment pour atteindre les impacts souhaités?

- Quels changements dans les pratiques et les comportements des acteurs sont nécessaires pour résoudre la problématique et atteindre les impacts souhaités?

- Qui devrait apprendre à faire quoi ? Qui devrait être capable de changer ses pratiques et comment ?

Une fois les changements souhaités identifiés, il faut comprendre quels sont les obstacles prévisibles qui s'opposeraient à ces changements, pourquoi tel ou tel acteur ne ferait pas ce qui serait souhaitable pour générer les impacts, quels éléments du contexte rendraient ces changements difficiles ou impossibles.

Des questions spécifiques peuvent aider à comprendre les obstacles au changement :

\section{Obstacles reliés aux acteurs}

- Les acteurs identifiés souhaitent-ils changer et pour quelles raisons ? En quoi le changement est en ligne avec leurs valeurs?

- Jusqu'à quel point les acteurs ont-ils les capacités, le savoir, les ressources disponibles, l'opportunité, le pouvoir de faire les choses différemment?

- Y a-t-il des acteurs ayant des intérêts qui peuvent rendre le changement difficile ou impossible ?

- Observe-t-on des rapports de force qui peuvent influer sur les changements souhaitables au niveau de certains acteurs?

- Pour quels changements souhaitables la recherche (ou les acteurs partenaires du projet) est-elle légitime et/ou en capacité de gestion et d'accompagnement?

Lors de la cartographie des résultats (outcomes], il est important de tenir compte des acteurs influents. Un acteur influent opposé à la solution proposée par l'intervention peut constituer un risque pour le succès de cette dernière (par exemple, une entreprise produisant des intrants qui se sentirait menacée par l'introduction d'une nouvelle pratique agricole). Si c'est le cas, il faut réfléchir à une stratégie en réponse à ces acteurs, à des activités de communication et de sensibilisation pour les convaincre, etc. Ce n'est pas forcément la recherche qui doit mener ces activités, mais il faut construire une stratégie anti-risques et identifier les acteurs partenaires qui doivent s'y impliquer.

Dans certains cas, c'est un élément de contexte qui pourra constituer un obstacle. Par exemple, en listant les obstacles, on peut trouver qu'une technologie proposée par l'intervention n'être appropriée que s'il y a un accès au crédit. Certains acteurs, comme des fournisseurs, pourraient avoir une attitude négative à l'égard de la technologie proposée si elle gêne leurs pratiques commerciales. 


\section{Obstacles reliés au contexte}

- Les conditions du milieu physique [sols, climat] ne contraignent-elles pas le changement ?

- L'environnement économique est-il favorable au changement (prix incitatifs, existence de marché, d'infrastructures, etc.) ?

- Y a-t-il un cadre réglementaire ou législatif qui encadre ou limite le changement ?

- La culture et les valeurs des acteurs du territoire ne définissent-elles pas des changements possibles et impossibles?

À nouveau, l'intervention seule ne pourra pas forcément traiter tous les obstacles identifiés : la réflexion devra se concentrer sur les obstacles majeurs que l'intervention peut surmonter. Pour les autres, l'intervention pourrait rechercher des liens avec d'autres projets ou interventions à même de les traiter. D'un autre côté, certains obstacles que l'intervention ne peut pas surmonter peuvent constituer des risques affectant l'appropriation des produits de recherche. Par exemple, une organisation et une culture bureaucratiques peuvent constituer un obstacle à la production d'un résultat. Surmonter de tels obstacles se situe souvent au-delà de l'objectif ou des possibilités de l'intervention ; dans ce cas, ces obstacles devraient être considérés comme des risques potentiels.

\subsection{Quelles stratégies pertinentes mettre en place avec les partenaires pour surmonter les obstacles?}

Une fois les obstacles identifiés, il s'agit de déterminer des stratégies pour les surmonter. La stratégie, c'est l'art de combiner ses ressources pour atteindre son objectif. II faut se concentrer sur les obstacles reliés aux acteurs, car ceux reliés au contexte demandent souvent des actions qui sont au-delà des capacités de l'intervention. La question est donc : comment faire pour que l'intervention contribue à l'usage, l'adaptation, ou l'appropriation de ses produits?

Les stratégies pour surmonter les obstacles peuvent être construites en se posant quelques questions :

- Si un acteur ne veut pas changer, peut-on influer sur son attitude ? Comment ? Qui peut le faire ?

- Si un acteur ne peut pas changer, comment peut-on lui faciliter l'accès à des ressources [cognitives, financières, matérielles, humaines) ?

- Faut-il proposer à un acteur de nouvelles technologies ou améliorer les technologies existantes ? Comment les expérimenter ou les tester avec les acteurs?

- La formation est certainement nécessaire pour certains acteurs afin de lever des obstacles, mais comment la penser? Qui peut la donner ? Avec quelle méthode ?

- La mise en place de nouveaux mécanismes de concertation entre deux acteurs permettra-t-elle de résoudre les conflits entre eux ? À quelles conditions ?

Une intervention ne pourra pas mettre en œuvre toutes les stratégies possibles, mais s'appuiera sur celles qui s'avèrent les plus susceptibles de se concrétiser en activités réelles. In fine, si les partenaires impliqués dans l'intervention n'ont pas les capacités, les moyens ou le mandat pour mettre en œuvre certaines stratégies, ils peuvent aussi envisager un accompagnement ou une intégration avec d'autres projets, programmes ou acteurs qui ont ces possibilités.

L'encadré 6 présente un exemple de réflexion sur les changements espérés, les obstacles identifiés et les stratégies pour surmonter ces derniers.

Cartographier puis relier entre eux les résultats, les obstacles, et les stratégies pour dépasser les obstacles, c'est in fine réfléchir à la façon de démontrer que les produits de l'intervention et les résultats génèrent effectivement des changements chez les acteurs. 
Afin de pouvoir adopter une nouvelle machine agricole, les agriculteurs doivent connaître son fonctionnement et être capables de l'utiliser ou de l'adapter, et ils doivent aussi pouvoir y accéder [coût acceptable, subvention, etc.) ; la machine peut générer des tensions au sein de la famille en accroissant le travail de certains ou auprès de salariés qui peuvent être exclus d'une opportunité de travail ; les agriculteurs peuvent être sceptiques par rapport à la nouvelle machine car elle heurte leur culture ; la machine doit être disponible [sur le marché ou par des actions publiques], et il ne doit pas y avoir de conflits entre les acteurs par rapport à sa distribution. Par exemple, les fournisseurs de machines concurrentes pourraient avoir une attitude négative par rapport à ce nouvel équipement.

Cette analyse de la situation amène à identifier des mesures à prendre :
- Une coordination entre la recherche et les services de vulgarisation sera peut-être nécessaire pour favoriser les interactions entre acteurs et l'adaptation de la machine en vue de son appropriation, ce qui signifie de nouveaux liens créés entre les acteurs, de nouvelles activités à prévoir pour l'intervention, etc.

- Pour la distribution de la nouvelle machine, il pourrait être nécessaire de développer de nouvelles stratégies commerciales, par exemple en incluant les fournisseurs de machines alternatives dans le jeu, en renforçant la capacité de négociation de certains acteurs, etc.

Ces actions peuvent se situer en dehors des possibilités d'action de l'intervention en cours de montage et dans certains cas, il sera donc nécessaire de développer des alliances avec d'autres interventions.

\subsection{Définir les activités, les moyens et les produits de l'intervention}

Jusqu'ici, les résultats et les acteurs qui en sont protagonistes, les obstacles aux changements souhaitables pour atteindre les résultats et les stratégies que l'intervention peut mettre en place pour surmonter ces obstacles ont été détaillés. En fait, les stratégies choisies représentent les grands axes d'activités que l'intervention mettra en place en totalité ou en partie. C'est à ce stade que peuvent être définis ou revus [s'ils avaient déjà été imaginés] les activités de l'intervention, et par conséquence les produits que l'intervention va générer.

Détailler les activités de l'intervention signifie défınir :

- le type d'intervention [analyse, expertise, formation, fourniture d'un service, etc.] ;

- la méthode choisie pour réaliser chaque type d'intervention, et notamment le degré de participation des acteurs dans la mise en œuvre de la méthode (recherche en situation d'observation, recherche-action, recherche participative, etc.] ;

- les interactions entre acteurs de l'intervention et hors intervention ;

- un chronogramme précis qui montre les activités par rapport au Gantt chart de l'intervention ;

- l'acteur responsable de l'activité et les acteurs qui participent à l'activité.

Les moyens (inputs) quant à eux concernent l'ensemble des ressources qui permettent de mener une activité (ressources humaines et matérielles, budget de recherche, informations, connaissances tacites ou autres, etc.) et ainsi de générer des produits (outputs) de recherche. Selon la démarche ImpresS ex ante, ces moyens (inputs) renvoient aux investissements réalisés et aux ressources mobilisées avant la période choisie pour le démarrage de l'intervention (un financement ou le recrutement d'un chercheur peuvent intervenir pendant la période de l'étude de cas et restent des inputs).

La définition des détails des activités dépend quand même du grain auquel le chemin de l'impact est construit : pour une intervention qui se concentre dans des zones et avec des acteurs spécifiques (et non des types d'acteurs), il est nécessaire de définir les activités et leur déroulement en détail. Dans ce cas-là, les stratégies apparaissent à un grain plus fin, davantage traduisibles directement en activités et produits [outputs] spécifiques [tableau 2]. 
Tableau 2 : Exemple de la déclinaison de stratégies à " grain fin » en activités et en produits [projet Maggi sur le clou de girofle à Madagascar].

\begin{tabular}{|l|l|l|}
\hline \multicolumn{1}{|c|}{ Stratégie à " grain fin " } & \multicolumn{1}{|c|}{ Activités } & \multicolumn{1}{c|}{ Produits [outputs] } \\
\hline $\begin{array}{l}\text { La fabrication locale d'alambics permet } \\
\text { d'assurer une production artisanale par les } \\
\text { paysans }\end{array}$ & $\begin{array}{l}\text { Mise au point d'un prototype d'alambic } \\
\text { Formation à la production et à l'usage } \\
\text { de l'alambic }\end{array}$ & $\begin{array}{l}\text { Alambic } \\
\text { Artisans locaux formés } \\
\text { Paysans formés }\end{array}$ \\
\hline $\begin{array}{l}\text { La certification foncière permet une gestion } \\
\text { durable du bois qui sert à alimenter les } \\
\text { alambics }\end{array}$ & $\begin{array}{l}\text { Formation sur la gestion durable dans } \\
\text { chaque site pour chaque acteur } \\
\text { Formation de pépiniéristes pour } \\
\text { reforester }\end{array}$ & $\begin{array}{l}\text { Certificats fonciers délivrés } \\
\text { Pépiniéristes formés }\end{array}$ \\
\hline $\begin{array}{l}\text { Des discussions approfondies avec Givaudan } \\
\text { distillerie industrielle) sur les approches vis- } \\
\text { à-vis des producteurs permettent de créer un } \\
\text { partenariat entre le privé et les paysans qui } \\
\text { distillent }\end{array}$ & $\begin{array}{l}\text { Rédaction d'un projet opérationnel pour } \\
\text { intéresser Civaudan }\end{array}$ & Projet opérationnel \\
\hline
\end{tabular}

Pour de grands programmes, des grappes de projets ou des dispositifs en partenariat, il est probable que les activités ne puissent pas être détaillées et que la défınition du chemin de l'impact hypothétique doive rester au niveau des stratégies et d'activités génériques. Au fur et à mesure que des projets plus précis s'inscrivent dans ces grands programmes, ces grappes de projets ou ces dispositifs, ou que des sites d'intervention spécifiques sont identifiés, il sera possible de définir les activités avec plus de détails.

Dans le cas où les activités sont issues des stratégies à un " grain plus gros " (tableau 3), elles peuvent même devenir les work packages de l'intervention.

Tableau 3 : Exemple de la déclinaison de stratégies à « gros grain » en activités et en produits (projet Wildmeat en Afrique centrale).

\begin{tabular}{|l|l|l|}
\hline \multicolumn{1}{|c|}{ Stratégie à " gros grain " } & \multicolumn{1}{c|}{ Activités } & \multicolumn{1}{c|}{ Produits [outputs] } \\
\hline $\begin{array}{l}\text { Les diagnostics participatifs des } \\
\text { filières permettent d'identifier les } \\
\text { points de blocage }\end{array}$ & $\begin{array}{l}\text { Atelier participatif et entretiens avec des acteurs } \\
\text { clés pour un diagnostic de chaque filière sur } \\
\text { chaque site }\end{array}$ & $\begin{array}{l}\text { Publications sur la base des } \\
\text { diagnostics } \\
\text { Actions participatives }\end{array}$ \\
\hline $\begin{array}{l}\text { Le renforcement des capacités } \\
\text { de gestion des acteurs leur permet } \\
\text { de mieux gérer les ressources de } \\
\text { leur territoire }\end{array}$ & $\begin{array}{l}\text { Actions de formation de formation à la gestion } \\
\text { dans chaque site pour chaque type d'acteurs en } \\
\text { s'appuyant sur des méthodes participatives } \\
\text { Création de comités locaux de gestion des } \\
\text { ressources }\end{array}$ & $\begin{array}{l}\text { Activités de formation } \\
\text { Identification des capacités à } \\
\text { renforcer par type d'acteurs } \\
\text { Comités locaux de gestion des } \\
\text { ressources }\end{array}$ \\
\hline $\begin{array}{l}\text { Négociation de contrats } \\
\text { d'exploitation durable des } \\
\text { ressources }\end{array}$ & $\begin{array}{l}\text { Discussions avec les acteurs publics et } \\
\text { privés pour définir le contenu d'un contrat } \\
\text { d'exploitation } \\
\text { Sessions de travail avec les comités de gestion } \\
\text { pour la négociation des contrats } \\
\text { Discussion de contrats dans les assemblées de } \\
\text { gouvernance locale }\end{array}$ & $\begin{array}{l}\text { Contenu d'un contrat d'exploitation } \\
\text { Contrats négociés et mis en œuvre }\end{array}$ \\
\hline
\end{tabular}

La réflexion sur les résultats, les obstacles et les stratégies pour dépasser ces derniers conduira probablement à identifier des activités complémentaires pour soutenir la génération des produits prévus ou la génération de nouveaux produits.

Construire le chemin de l'impact ex ante sur la base du processus itératif nécessite donc à ce stade de retourner au récit pour:

- expliciter les résultats que l'intervention souhaite obtenir par la génération des produits et les stratégies retenues pour y parvenir ;

- revoir la plausibilité des impacts sur la base des résultats [outcomes] identifiés avec leurs liens de causalité. 
Pour construire une vision commune, il peut être intéressant de travailler sur une structure de récit, représentée sous forme d'un graphe. Cela est surtout utile quand on commence l'exercice de réflexion ex ante après soumission d'une proposition ou d'une concept note, par exemple lors de l'atelier de démarrage de l'intervention.

Il s'agit de retrouver dans la proposition les éléments constitutifs du récit tels que les suggère la démarche Impress, et de les organiser sous forme de récit court, clair et convaincant. Une fois que les éléments disponibles sont repérés et structurés dans un récit, on présente la relecture de la proposition pendant l'atelier de démarrage à l'équipe de l'intervention, ses partenaires et possiblement les acteurs majeurs. Cette relecture peut prendre différentes formes, mais deux supports sont considérés comme utiles : l'envoi aux participants du récit par l'équipe projet avant l'atelier de démarrage pour entamer une discussion; et la représentation graphique des éléments clés de l'intervention. Cette dernière, utile pour lancer un débat, montre dans quel[s] chemin[s] de l'impact global[aux] s'inscrit l'intervention en explicitant les impacts et les résultats [outcomes], et en montrant les work packages prévus pour organiser le travail. On peut ainsi représenter sur la même visualisation deux façons de voir l'intervention : selon les mécanismes de causalité et selon la logique projet.

En fait, cet exercice de relecture et de visualisation a pour but de générer un débat autour des éléments clés de l'intervention, pour pouvoir se les approprier, les transformer et finalement arriver à une vision partagée.

La figure montre un exemple de visualisation après relecture de la proposition du projet TAMA.

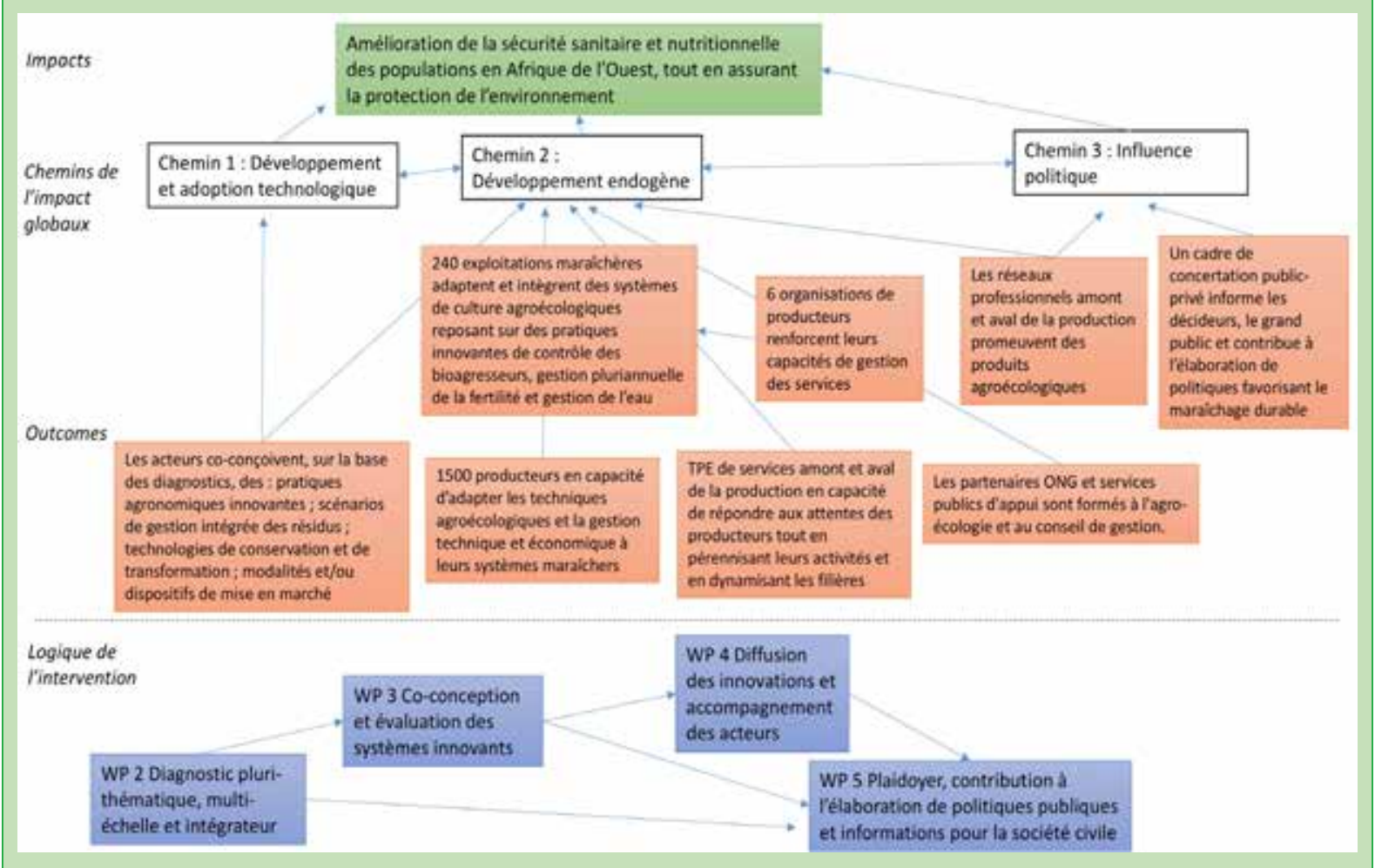




\section{Étape 3. Prendre en compte les politiques publiques}

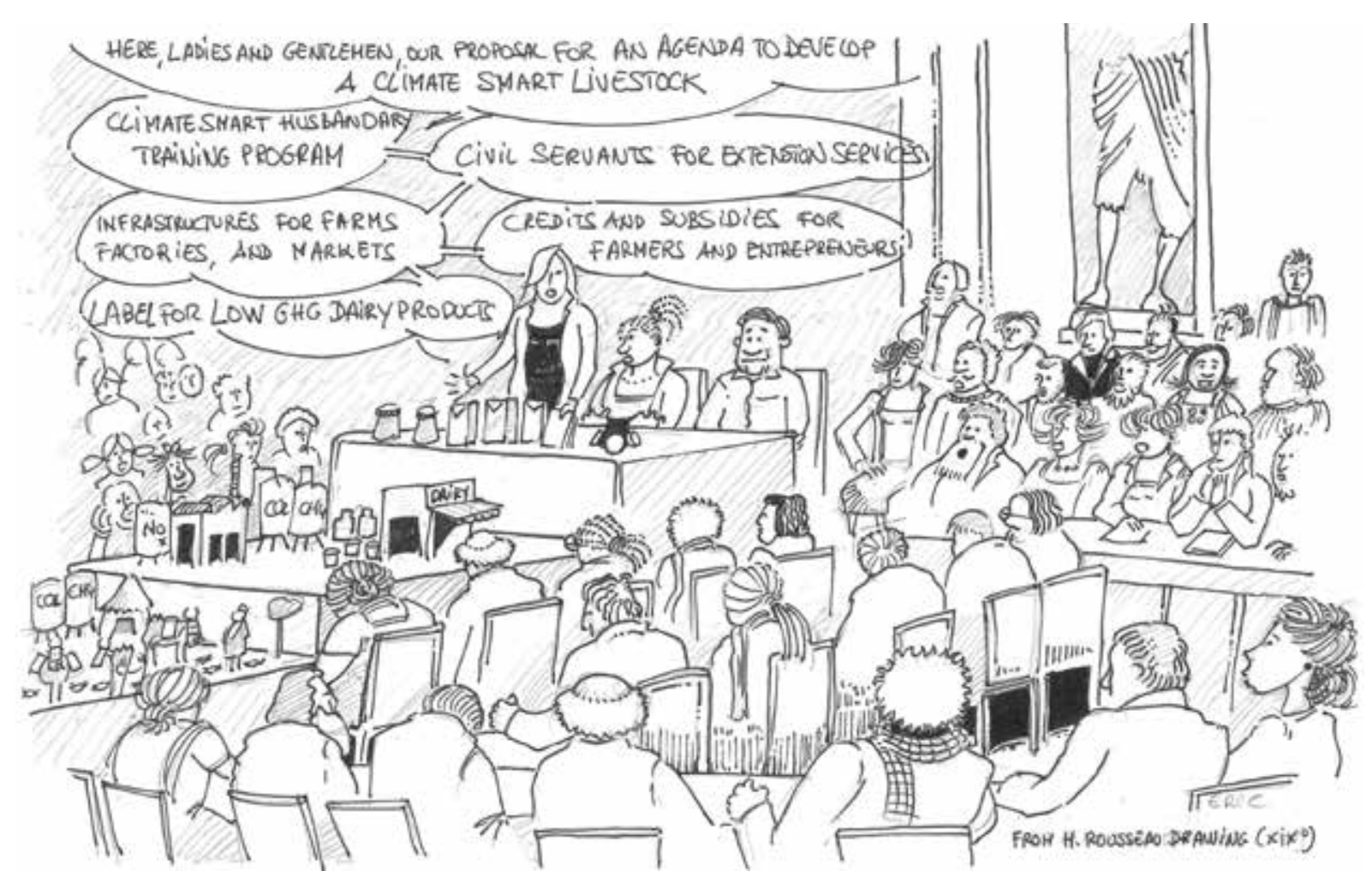

Dans la réflexion sur les résultats (outcomes) et les changements majeurs, il est nécessaire de s'intéresser plus particulièrement à deux aspects analysés dans le cadre de la démarche ImpresS ex post : les interactions avec les acteurs des politiques publiques et le renforcement des capacités des acteurs. Même si ces aspects ont déjà été abordés dans les étapes précédentes, deux étapes spécifiques de ce chapitre leur sont dédiées, du fait de leur importance.

\subsection{Pourquoi faut-il prendre en compte les politiques publiques ?}

Le contexte institutionnel a une grande influence sur le processus d'innovation. Une des leçons tirées de la démarche ImpresS ex post est que les interactions avec les acteurs des politiques publiques sont nécessaires pour développer l'innovation et générer des impacts. Les interactions entre la recherche et les politiques publiques sont déterminantes pour permettre à la recherche de contribuer à des impacts sociétaux. Les recherches s'inscrivent dans un contexte institutionnel, façonné par les politiques publiques passées et présentes, plus ou moins porteur et favorable à l'innovation. L'émergence de certaines priorités dans les agendas politiques, sur le long terme ou en réaction à une crise, peuvent orienter les choix et les modes d'intervention des chercheurs et la capacité de leurs recherches à avoir de l'impact. Les acteurs des politiques publiques, par des moyens très diversifiés, ont un effet de levier qui rend l'impact de la recherche possible. En interagissant avec les acteurs publics, et c'est particulièrement le cas des recherches participatives qui en associent certains, les chercheurs jouent ainsi un rôle dans l'élaboration des politiques publiques. Mais quand l'objet de la recherche n'est pas directement lié aux acteurs publics, les chercheurs décident plus rarement de communiquer avec ceux-ci ou de les solliciter. Enfin, la réceptivité des acteurs publics vis-à-vis des travaux des chercheurs peut être faible dans le contexte des pays en développement.

Les acteurs publics peuvent jouer un rôle clé à différentes étapes du chemin de l'impact, que ce soit en influençant directement l'orientation de la recherche, en orientant les financements et les subventions pour les acteurs qui innovent, en produisant des règles ou des normes ou en orientant les organisations en charge de la formation. Ils ont par ailleurs un rôle crucial dans les étapes de changement d'échelle. 
La participation des acteurs publics aux processus d'innovation, et en particulier leur collaboration à des recherches participatives multi-acteurs, renforcent leurs capacités à interagir avec les chercheurs et les autres acteurs du système d'innovation et à faciliter durablement l'impact des recherches.

La recherche impacte aussi les politiques publiques en rédigeant des plaidoyers, en participant à la construction ou à l'évaluation des politiques publiques. Cependant, les agendas politiques diffèrent des agendas scientifiques. Les chercheurs sont appelés à être flexibles pour interagir avec les acteurs publics, par exemple via des relations informelles, ou en participant à des coalitions visant à influer certaines politiques publiques qui sont favorables à l'innovation.

\subsection{Comment repérer et renforcer les interactions avec les acteurs publics ?}

Quand on définit à la première étape [voir partie "Étape 1 : Qui sont les acteurs majeurs, influents et impactés de l'intervention ? "] les acteurs majeurs, influents et impactés et les changements majeurs souhaités, il est important de considérer à ce stade plus particulièrement les acteurs publics qui contribuent à la conception ou à la mise en œuvre de politiques publiques aux échelles nationale ou locale. Un acteur public peut être défini comme un acteur majeur, un acteur influent, et/ou un acteur impacté selon le processus d'innovation analysé. Les études de cas analysées dans le cadre de la démarche ImpresS ex post aident à repérer les différents types d'acteurs publics :

- les acteurs publics nationaux [État, ministères, services centraux, etc.] ;

- les services déconcentrés de l'État [administration, préfecture / sous-préfecture, etc.] qui sont le relai des acteurs publics nationaux ;

- les acteurs publics locaux des collectivités locales (acteurs indépendants administrativement et politiquement de l'État, même s'ils sont en grande partie financés par l'état, comme des régions ou des communes] ;

- les organisations internationales et autres acteurs publics extérieurs au pays où se déroule l'intervention (bailleurs de fonds, coopération bilatérale ou multilatérale, etc.), qui mènent leurs propres stratégies politiques et influencent les politiques des gouvernements dans les pays en développement.

Lors de la cartographie des résultats [outcomes], ceux reliés aux acteurs publics doivent clairement être identifiés. On doit également préciser le moment auquel l'intervention d'un acteur public dans le processus d'innovation est attendue et le/les rôles qu'il serait conduit à jouer pour favoriser l'impact.

Il est nécessaire d'être attentif aux modalités concrètes de l'action publique et aux moments auxquels les acteurs publics sont susceptibles d'entrer en relation avec les autres acteurs de l'innovation et de faciliter (ou freiner] le processus menant à l'impact. Ainsi, les acteurs publics peuvent avoir un effet de levier sur l'innovation [création ou changement d'échelle) au cours des différentes phases du chemin de l'impact :

- au niveau des investissements pour la recherche [inputs] : financement public, orientations des programmes de recherche, mise en relation avec d'autres acteurs, etc. ;

- au niveau de la production de la recherche [outputs] : contribution des acteurs publics à des recherches participatives multi-acteurs ; création d'un cadre propice à l'innovation ;

- au niveau de la création de résultats (outcomes) : mobilisation d'acteurs, mise en place de normes et de règles, création de structures de concertation ou de gestion, financement d'opérations de communication, financement d'investissement, etc. ;

- au niveau des impacts : incitations financières, création d'un environnement favorable à l'innovation, facilitation du changement d'échelle, etc.

Cette réflexion a déjà été conduite dans l'étape 2, mais faire un point particulier sur les interactions avec les politiques publiques permet de ne pas omettre certaines activités ou stratégies indispensables pour obtenir des ouputs et outcomes. Réfléchir aux interactions avec les acteurs publics amène à revoir les résultats [outcomes] et les changements que l'on souhaite accompagner. Cette boucle implique aussi un enrichissement du récit. 


\section{Étape 4. Viser le renforcement des capacités}

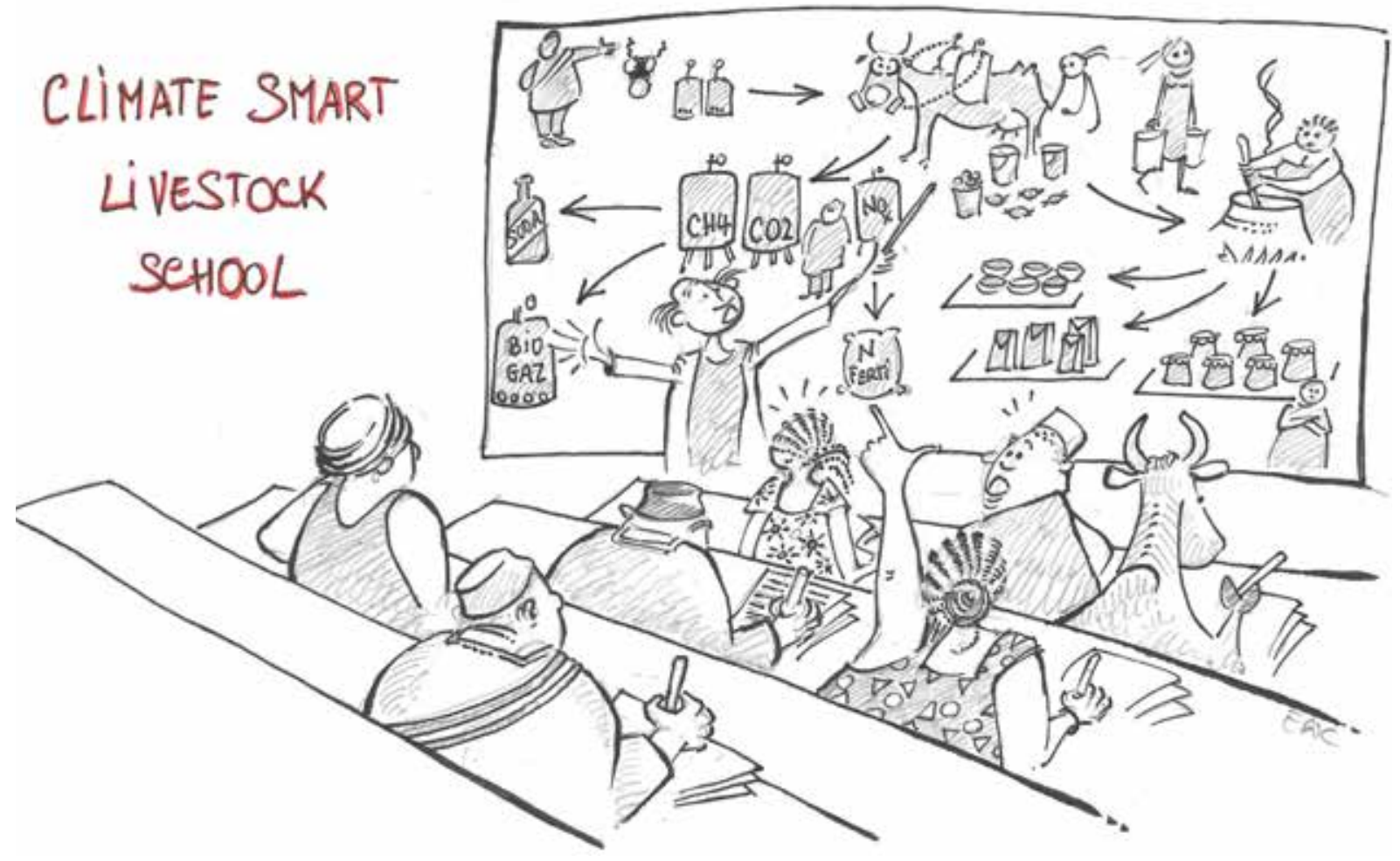

\subsection{Pourquoi prendre en compte le renforcement des capacités ?}

Les changements de pratiques et de comportements se produisent parce que des individus, des communautés ou des organisations acquièrent ou développent de nouvelles capacités et compétences. Le Cirad, en déployant ses activités de recherche en partenariat, contribue au renforcement des capacités de ses partenaires, dans le but de permettre aux chercheurs du Sud et à leurs institutions, mais aussi aux acteurs du développement (agriculteurs, représentants des organisations de producteurs, agents techniques des ONG, responsables d'entreprises privées, cadres des institutions publiques], de mieux répondre aux défis du développement.

Le renforcement des capacités implique un processus d'acquisition et d'accumulation de connaissances, de savoirs, de savoir-faire et de savoir-être, suivi d'une application par les individus ou organisations pour remplir leurs fonctions et atteindre les objectifs visés (Morgan, 1998). Le renforcement des capacités se fait dans l'action, dans le cadre de situations d'apprentissage. Une situation d'apprentissage (Toillier, 2012) est définie comme un ensemble de conditions et de circonstances susceptibles d'amener une personne, un collectif ou une organisation à construire des nouvelles connaissances et à les appliquer pour résoudre des problèmes, saisir des opportunités ou améliorer des façons de faire. L'apprentissage résulte en de nouvelles capacités. Une situation d'apprentissage peut être organisée ou informelle, intentionnelle ou non.

Dans une étude de cas issue de l'analyse ImpresS ex post, le "Riz pluvial d'altitude à Madagascar ", où la recherche intervenait sur le mode " transfert participatif ", plusieurs situations d'apprentissage formelles ont été identifiées. Par exemple, les techniciens vulgarisateurs et les partenaires de la recherche ont participé à des formations organisées par l'équipe de recherche sur la culture et les techniques culturales et sur les capacités pour les vulgariser.

\subsection{Quel type de renforcement des capacités et pour quels acteurs ?}

Dans la réflexion ImpresS ex ante, le renforcement des capacités des acteurs majeurs et des acteurs impactés constitue un mécanisme clé pour atteindre les résultats [outcomes] et obtenir les impacts. Le 
renforcement des capacités recouvre le renforcement du capital humain (individus) et du capital social (organisations, relations entre individus ou organisations à travers des réseaux formels ou informels). Les capacités à renforcer sont très diverses - techniques, managériales, capacités à expérimenter, à apprendre, à interagir avec les autres - en fonction des innovations spécifiques en cours de développement. Prises ensemble, elles permettent aux acteurs concernés de développer une plus grande capacité à innover [tableau 4].

Tableau 4 : Types de capacités définies dans les études de cas ImpresS.

\begin{tabular}{|c|c|c|c|c|}
\hline $\begin{array}{l}\text { Capacités } \\
\text { techniques }\end{array}$ & $\begin{array}{l}\text { Capacités } \\
\text { de gestion }\end{array}$ & $\begin{array}{l}\text { Capacitēs } \\
\text { à expérimenter } \\
\text { et à apprendre }\end{array}$ & $\begin{array}{l}\text { Capacités à interagir } \\
\text { avec les autres }\end{array}$ & $\begin{array}{c}\text { Capacités dont } \\
\text { le renforcement conforte } \\
\text { le pouvoir d'agir }\end{array}$ \\
\hline $\begin{array}{l}\text { Maîtriser } \\
\text { une nouvelle } \\
\text { technologie } \\
\text { Maîtriser de } \\
\text { nouveaux } \\
\text { procédés }\end{array}$ & $\begin{array}{l}\text { Savoir analyser sa } \\
\text { situation et son } \\
\text { environnement } \\
\text { Savoir programmer ses } \\
\text { activités } \\
\text { Savoir suivre et évaluer } \\
\text { ses activités et résultats } \\
\text { Savoir mobiliser des } \\
\text { ressources [financières } \\
\text { et autres] } \\
\text { Savoir gérer son } \\
\text { exploitation et évaluer } \\
\text { la performance des } \\
\text { innovations au regard de } \\
\text { leurs critères [diagnostic] }\end{array}$ & $\begin{array}{l}\text { Savoir expérimenter, } \\
\text { adapter } \\
\text { Savoir formaliser des } \\
\text { connaissances pour } \\
\text { résoudre d'autres } \\
\text { problèmes } \\
\text { Savoir partager les } \\
\text { connaissances et } \\
\text { compétences avec ses } \\
\text { pairs et autres acteurs }\end{array}$ & $\begin{array}{l}\text { Savoir travailler } \\
\text { ensemble pour concevoir } \\
\text { et mettre en œuvre une } \\
\text { innovation } \\
\text { Savoir agir } \\
\text { collectivement pour } \\
\text { concevoir et mettre en } \\
\text { œuvre une organisation } \\
\text { et s'engager dans un } \\
\text { processus politique } \\
\text { Savoir interagir avec } \\
\text { les autres acteurs du } \\
\text { système d'innovation } \\
\text { [État, entreprises, } \\
\text { marchés, etc.) }\end{array}$ & $\begin{array}{l}\text { Prendre confiance en soi } \\
\text { Modifier sa perception } \\
\text { d'un problème et des } \\
\text { solutions } \\
\text { Devenir pro-actif } \\
\text { Augmenter le pouvoir } \\
\text { de décision et la } \\
\text { participation des } \\
\text { femmes ou des groupes } \\
\text { marginalisés dans les } \\
\text { systèmes d'innovation }\end{array}$ \\
\hline
\end{tabular}

\subsection{Comment repérer les besoins de renforcement des capacités ?}

Il est important réfléchir dans le contexte ex ante aux moyens de promouvoir les situations d'apprentissage qui permettront le renforcement des capacités. Ce dernier peut intervenir tout au long du chemin de l'impact et constituer un prérequis à la génération des produits de la recherche, par exemple quand la recherche co-conçoit les produits avec les paysans. Il peut aussi constituer une stratégie pour dépasser des obstacles et atteindre des résultats [outcomes] : par exemple, dans le transfert participatif de technologies, les paysans ne peuvent pas adopter une variété sans formation sur sa gestion agronomique.

Dans le cadre de l'évaluation ex ante, il faut identifier les besoins en renforcement des compétences par type d'acteurs pour les outputs et pour les outcomes, mais aussi les situations d'apprentissage à favoriser en se posant les questions suivantes:

- Quels sont les besoins de renforcement des capacités des agriculteurs, des leaders paysans, des techniciens, des entreprises, etc. ?

- Quelles sont les méthodes les plus appropriées pour ce renforcement des capacités (formation en salle, échanges entre pairs, visite de terrain, essais chez les paysans, accès à des informations, etc.) ?

- Quels sont les moments les plus appropriés pour mener ces activités de renforcement des capacités ?

- Qui peut accompagner le processus de renforcement des capacités (chercheur, technicien, éducateur, agriculteurs ayant un savoir particulier, etc.) ?

- La recherche a-t-elle les capacités pour concevoir et participer à ce renforcement des capacités ? Peutelle ou doit-elle renforcer ses propres capacités pour agir ?

Cette réflexion a normalement déjà été conduite au cours de l'étape 2, mais faire un point particulier sur le renforcement des capacités permet de ne pas omettre des activités ou stratégies indispensables pour obtenir des ouputs et outcomes. Finalement, comme dans le cas des interactions avec les acteurs publics, réfléchir au renforcement des capacités conduit à enrichir le récit. 


\section{Étape 5. Finaliser le chemin de l'impact et imaginer des chemins de l'impact alternatifs}

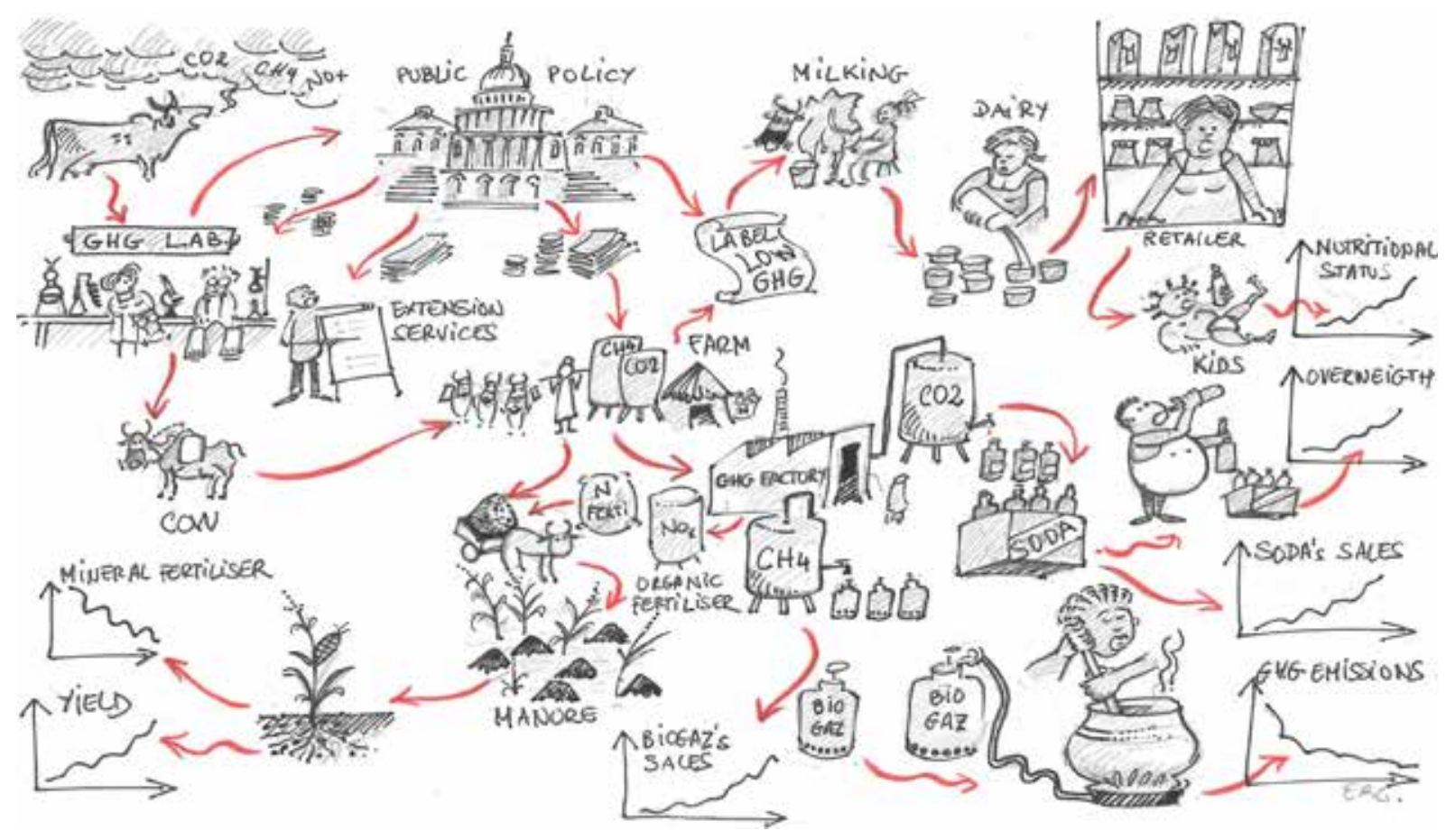

\subsection{Dessiner le chemin de l'impact principal}

À cette étape, tous les éléments pour proposer le chemin de l'impact argumenté de l'intervention sont disponibles: les impacts, les outcomes, les outputs, et les inputs. Il faut retourner au récit de l'intervention, à la cartographie des résultats [outcomes], à l'identification des stratégies pour relier les cases du chemin de l'impact. Ces liens représentent les relations de causalité entre produits, résultats et impacts.

Les stratégies choisies aident à expliciter les liens de causalité, $y$ compris celles reliées aux acteurs publics et au renforcement des capacités. Par exemple, pour expliquer le lien entre la production d'un alambic (produit) et l'utilisation par les artisans [résultat), l'explicitation du lien de causalité est la " co-conception et fabrication locale de l'alambic » et la « formation / conseil auprès des agriculteurs ». On peut rendre plus visibles les activités de renforcement des capacités en soulignant les situations d'apprentissage critiques sur le chemin de l'impact, par exemple avec une étoile (figure 10, page suivante).

\subsection{Identifier les facteurs contextuels influençant le chemin de l'impact}

Le contexte institutionnel est un facteur important à prendre en compte pour comprendre un processus d'innovation et son déroulement. Il faut donc apprécier le contexte institutionnel dans lequel l'intervention s'insère et qui pourra avoir une influence sur la mise en œuvre et l'impact de l'intervention.

À cette étape, il s'agit de réfléchir aux facteurs de contexte qui influencent le processus d'innovation, y compris ceux qui sont internes; la composition du partenariat de l'intervention, le niveau de collaboration entre les acteurs, les acteurs leaders, l'accès à des financements additionnels, etc., qui sont liés au montage de l'intervention, peuvent en effet faciliter ou rendre plus difficile le chemin de l'impact recherché dans le futur. II faut identifier les facteurs de contexte connus ou attendus qui pourraient favoriser / empêcher les changements souhaités et constituent ce qu'il est convenu d'appeler l'« enabling environment " (cf. Douthwaite et al., 2017). Les acteurs ont souvent des idées sur la façon dont leur contexte peut changer dans les années à venir, compte-tenu des tendances de l'économie, des nouvelles lois, etc., mais il peut aussi exister des études prospectives, des plans stratégiques nationaux qui projettent les priorités de l'action publique à 5 ans, à 10 ans, etc. Il est nécessaire de prendre en compte l'existence de tels documents ou études. L'intervention peut d'ailleurs contribuer à modifier ces facteurs ou se contenter de les considérer comme des risques ou des opportunités. 
Input

Les équipes

de lintervention

ont mobilise

ces ressources

\section{Output \\ Outcome}

Pour générer ces produits de Tintervention
Les acteurs se sont saisis

des produits et les ont transformees pour se les approprier...
Impact 1

Ces résultats ont provoqués des impacts chez les acteurs qui interagissent directement ou indirectement avec rintervention...
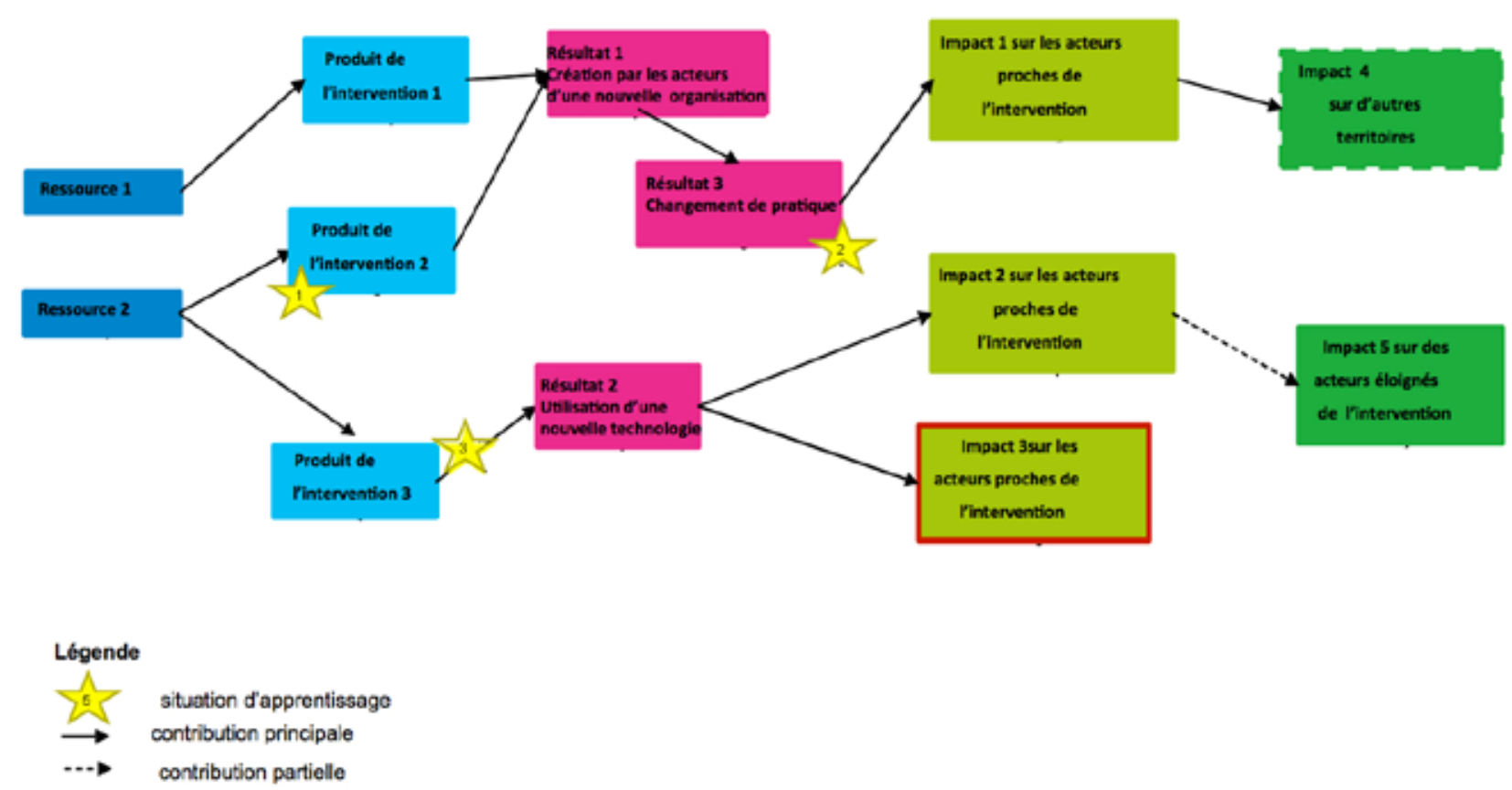

Figure 10 : Visualisation schématique du chemin de l'impact avec mise en évidence des situations d'apprentissage.

Parmi les facteurs contextuels à considérer, peuvent par exemple être citées les politiques publiques ou privées suivantes :

- les politiques scientifiques, de recherche, d'innovation, de formation, qui conditionnent les moyens humains, techniques et financiers affectés à la recherche ;

- les investissements planifiés par le secteur privé dans l'agriculture ;

- l'agenda public fixant les priorités des questions à traiter ;

- les politiques de développement ou sectorielles, en appui desquelles les projets de recherche interviennent directement : politiques agricoles, politiques du crédit, politiques alimentaires, politiques sanitaires, politiques foncières, politiques énergétiques, etc.

Par exemple, si le chemin envisagé pour augmenter durablement la productivité agricole passe par la mise en œuvre des techniques agroécologiques, les facteurs favorables peuvent inclure l'accès et la disponibilité de moyens de communication, l'existence d'une loi en faveur de l'agroécologie, et la disponibilité de financements internationaux. Inversement, les facteurs susceptibles de gêner le changement souhaitable peuvent inclure des politiques de subvention aux intrants chimiques, des politiques foncières ne favorisant pas la stabilité de l'accès à la terre, ou la promotion continue par de grandes entreprises de technologies non compatibles avec celles proposées, etc.

Autre exemple, si un chemin de l'impact qui repose sur des concertations et prises de décision participatives pour gérer les ressources naturelles est proposé : les facteurs favorables dans ce contexte incluraient les politiques favorisant la décentralisation, ou instituant et rendant obligatoires certains types de consultations des acteurs de la société civile, alors que des facteurs défavorables seraient des règles et routines centralisées et verticales de prise décision publique, ou l'absence de financement accordé aux collectivités, des modes paternalistes de prise de décision, etc. 


\subsection{Imaginer des chemins de l'impact alternatifs}

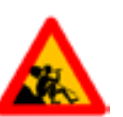

Souvent, plusieurs chemins de l'impact plausibles peuvent être envisagés, et cela pour diverses raisons. Des facteurs de contexte peuvent être valorisés ou pondérés différemment par différents acteurs impliqués dans l'intervention, certains accordant une influence forte à certains facteurs, d'autres non. Il peut aussi y avoir différentes préférences de mode d'action, certains acteurs voulant privilégier les approches plus verticales, les considérants comme plus efficaces, d'autres les approches plus concertées. En fonction de ces différentes lectures des contextes, et des risques ou opportunités associés, il peut y avoir des façons différentes d'envisager les interventions à mener et les meilleurs moyens d'obtenir les résultats recherchés.

Sur cette base, il est possible d'imaginer facilement ex ante deux ou trois chemins de l'impact aussi plausibles les uns que les autres. Pour ce faire, il faut identifier deux ou trois résultats [outcomes] essentiels à la contribution de l'intervention à l'impact et réfléchir à une, voire deux stratégies alternatives, différentes de celles envisagées dans la partie « Étape 2 : Définir les activités, les moyens et les produits de l'intervention ", et qui pourraient être mises en place pour atteindre ces résultats d'une manière considérée comme plausible sur la base de raisonnements explicites. Il s'agit de répondre à la question : quelle[s] autre[s] stratégie[s] pourrai(en]t être mise[s] en œuvre pour atteindre ce même résultat, et aboutir à ces mêmes impacts ? Dans certains cas, cela peut amener l'équipe ex ante à réfléchir non seulement à différentes façons d'atteindre des résultats [outcomes], mais aussi à d'autres produits [outputs] à développer, qui serviront de base à l'obtention des résultats de ces différents scénarios.

In fine, on obtient le chemin de l'impact principal et un, deux ou trois chemins alternatifs qui peuvent être suivis durant la mise en œuvre de l'intervention.

Il peut s'avérer utile de s'appuyer sur des études prospectives portant sur la problématique centrale de l'intervention pour nourrir la réflexion sur les divers scénarios possibles. Les méthodes de prospective permettent d'identifier des grandes tendances et d'imaginer différents futurs par rapport à une situation donnée. Mais ces méthodes ne détaillent pas le comportement de chaque acteur par rapport à la production d'un résultat ou pour surmonter un obstacle.

À ce stade, tous les éléments pour compléter le récit afin qu'il reflète l'histoire que le chemin de l'impact permet de visualiser sont disponibles. Le récit sera convaincant et plausible et il communiquera la vision partagée des partenaires pour les mobiliser vers un objectif commun, en intégrant le cas échéant des scénarios alternatifs.

Reprendre à ce moment le chronogramme (voir partie « Étape 1: Un chronogramme provisoire à compléter à la fin de la réflexion "] permet d'imaginer le temps approximatif de production des résultats [outcomes]. 


\section{Étape 6. Suivi-évaluation participatif et apprentissage}

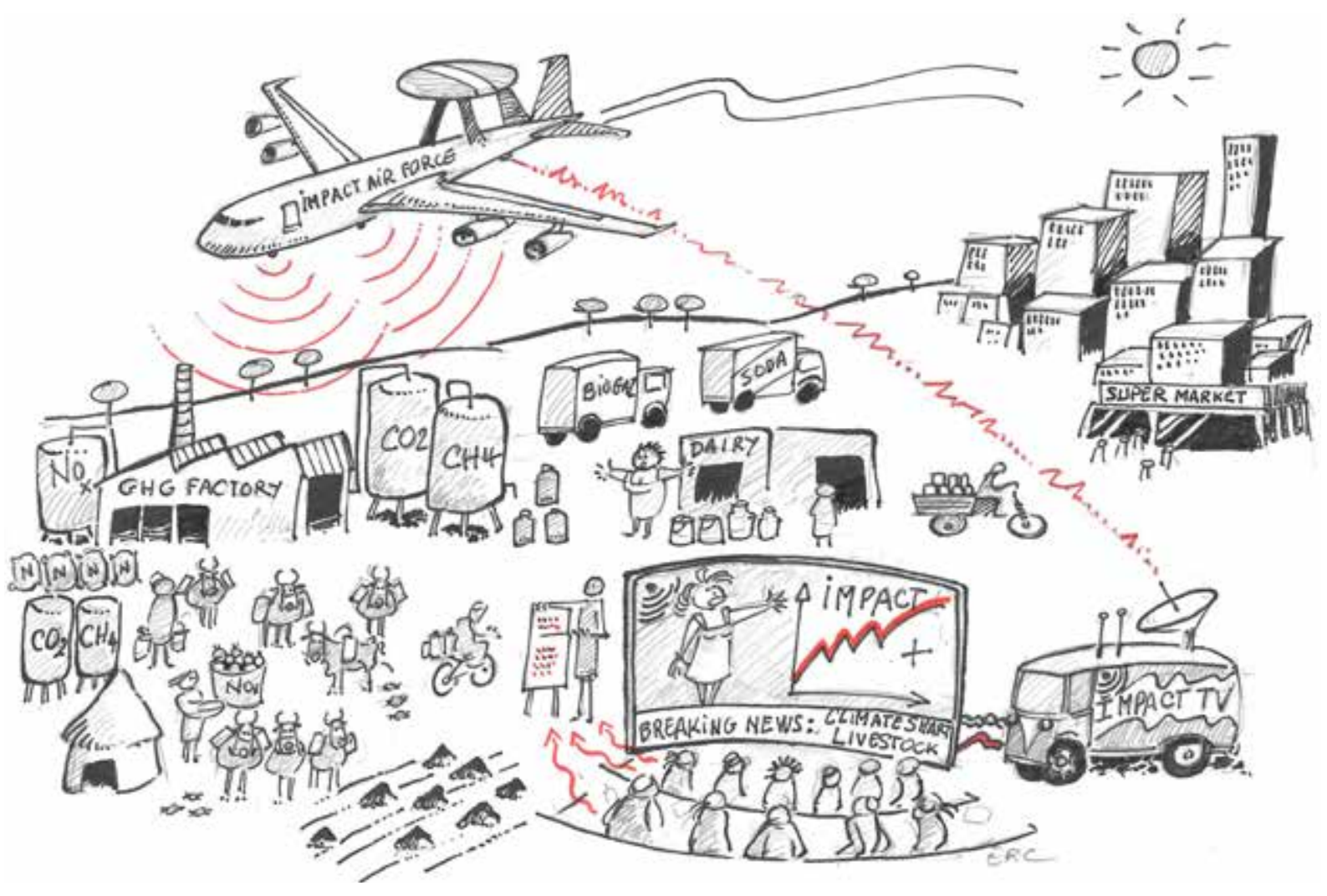

La démarche ImpresS ex ante se trouve encore dans une phase exploratoire par rapport à l'étape suivi-évaluation participatif et apprentissage [SEPA ou PMEL ${ }^{10}$ ]. À ce stade, cette partie se contente de proposer quelques pistes générales sur le SEPA; elle fera l'objet de développements méthodologiques futurs.

Les bailleurs se concentrent généralement sur le suivi des produits d'un projet et ne regardent que peu les résultats (outcomes]. Ils ne prennent pas en considération la consolidation des produits et des résultats obtenus par une grappe de projets. La démarche ImpresS ex ante vise à suivre les progrès accomplis dans l'obtention des résultats (outcomes] et des apprentissages correspondants, en prenant en considération le temps long (grappe de projets). II s'agit d'un suivi finalisé, favorisant le pilotage adaptatif de l'intervention.

L'équipe ImpresS préconise que ce suivi soit participatif. La FAO définit le suivi participatif ${ }^{11}$ comme " having all passengers on the bus know their destination and decide how they will measure their progress " (le fait que tous les passagers du bus connaissent leur destination et décident comment mesurer le progrès vers cette destination]. Sur cette base, il est clair que développer un système SEPA et le mettre en œuvre impliquent forcément la participation active des acteurs majeurs de l'intervention, passagers mais aussi chauffeurs du bus pour certains d'entre eux. Et cela implique aussi que la destination du bus (l'impact recherché] ait été validée, voire construite, par tous.

Dans cette section, sont abordés l'identification des indicateurs, le déploiement du SEPA, et l'utilisation de ses résultats pour piloter l'intervention.

10 PMEL : sigle anglais pour «Participatory monitoring evaluation and learning ».

11 www.fao.org/docrep/x5307e/x5307e05.htm 


\subsection{Les indicateurs : de quel type ? Avec quelles caractéristiques ? Mesurés par qui ?}

Sur la base des objectifs communs identifiés pour l'intervention (résultats et impacts), il s'agit donc d'identifier ou de développer, si possible de manière participative [Lennie et al., 2011), deux types d'indicateurs : des indicateurs de suivi, qui documentent le progrès de l'intervention vers les résultats et permettent de rendre compte de la nature du processus d'innovation en cours, et des indicateurs finaux, qui montrent si les résultats ont été obtenus. Il faut également que ces deux types d'indicateurs permettent non seulement de suivre les changements en cours, mais aussi de déterminer pourquoi et comment ceux-ci sont ce qu'ils sont : c'est en grande partie sur la réponse à ces dernières questions que reposent les possibilités d'apprentissage collectif et de pilotage.

Bien que les indicateurs s'intéressent surtout au suivi des résultats et des processus d'innovation permettant de les obtenir, il est important de définir aussi des indicateurs permettant de suivre les évolutions clés des facteurs de contexte, car cela peut amener l'équipe en charge de l'intervention à privilégier certains scénarios envisagés lors de la réflexion ex ante plutôt que d'autres, ou à mieux interpréter les progrès (ou leur absence) observés.

Finalement, il faut que les indicateurs sur lesquels l'équipe en charge de l'intervention se mettra d'accord restent limités en nombre et suffisamment " simples " à interpréter, afın de ne pas causer une surcharge de travail et un coût excessifs pour leur collecte et analyse périodique, qui se feraient au détriment de la mise en place des activités. Autrement dit, le SEPA ne peut pas avoir vocation à suivre de manière exhaustive et détaillée les progrès, les résultats obtenus ou l'évolution du contexte, mais doit plus modestement permettre de détecter les problèmes lors de la mise en œuvre et offrir des clés de compréhension et d'ajustement.

Dans certaines situations, par exemple dans un contexte nouveau ou en travaillant avec de nouveaux acteurs, il peut être intéressant de se garder une certaine souplesse, de ne pas verrouiller un SEPA et d'attendre que le processus d'innovation prenne corps pour identifier les indicateurs des résultats [outcomes].

Par exemple, dans le projet Sustainable Wildlife Management en Zambie, le résultat (outcome) suivant a été identifié : « Des communautés de conservation ont été établies et gèrent et commercialisent la faune sauvage ». Des indicateurs de progrès pourraient concerner la formation et l'évolution des communautés de conservation (nombre, type, acteurs participant ou s'opposant, formalisation des relations entre acteurs, etc.) et l'établissement de règles de gestion, alors que les indicateurs finaux pourraient concerner les résultats de gestion (nombre et type de contrats, acteurs participant, résolution de conflits, environnement [état des ressources naturelles] et les résultats de commercialisation [nombre d'échanges, acteurs participant, quantités mises sur le marché) de faune sauvage.

Le choix des indicateurs dépend aussi en partie de qui sera en charge de la mise en œuvre du SEPA, et donc de la stratégie de collecte des indicateurs : par exemple, un indicateur pour suivre l'évolution des rendements d'une culture ne sera pas forcément le même si c'est une organisation de producteurs qui est chargée de son suivi et peut vouloir privilégier les indicateurs qualitatifs visuels (aspect des cultures, grosseur des épis], ou si c'est un chercheur qui tendra à privilégier un indicateur plus quantitatif basé sur des mesures (kg / ha).

Les méthodes participatives de collecte de données pour renseigner les indicateurs sont souvent recommandables, même si elles ne sont pas toujours obligatoires et peuvent avoir des désavantages [coûts, difficulté de standardisation et de comparaison, etc.) : elles tendent cependant à accroître l'apprentissage collectif sur la progression de l'intervention et l'émergence de consensus sur les nécessités éventuelles d'ajustements dans les stratégies mises en œuvre ; elles favorisent également l'appropriation des résultats par les acteurs. Elles sont logiques dans les cas de co-conception. Dans un tel contexte, le SEPA peut vouloir privilégier la collecte effectuée lors d'ateliers multi-acteurs, ou dans des focus groups par type d'acteurs permettant de recueillir des informations désagrégées, plutôt que la collecte via un travail analytique moins facilement partageable que mèneraient des chercheurs ou des techniciens. Les ateliers de lancement d'une intervention sont une bonne occasion pour planifier et initier ce travail participatif sur les indicateurs. Durant la phase de conception ex ante du chemin de l'impact, travailler sur les indicateurs 
de suivi des résultats peut être particulièrement utile si les acteurs pertinents pour définir le suivi sont présents.

Le « Equal Access Participatory Monitoring and Evaluation toolkit » ${ }^{12}$ donne des recommandations pour la définition participative d'indicateurs de suivi.

\subsection{Mettre en œuvre et utiliser les résultats du SEPA}

Le but du suivi des résultats (outcomes) via le SEPA est de favoriser les apprentissages collectifs et de permettre des réévaluations et ajustements participatifs réguliers du chemin de l'impact initial en cas de besoin. Les données issues du SEPA doivent permettre de s'assurer que l'intervention contribue effectivement à l'impact, plutôt que de fournir un suivi mécanique et rigide d'un plan de travail pré-établi. Cette dernière attitude pourrait facilement conduire à des dérives dommageables, voire des échecs, pour différentes raisons : erreur ou omission sur les facteurs de contexte pris en compte, ou sur la lecture initiale des obstacles, non-obtention de certains produits invalidant du même coup le chemin de l'impact qui les intègre, dynamique imprévue du partenariat, etc.

Dans cette vision, le SEPA peut et doit permettre d'interagir avec les partenaires de l'intervention et d'autres acteurs, afin de tirer des enseignements de l'intervention en cours, d'évaluer le processus d'innovation et d'aider les acteurs à adapter leurs activités et actions, notamment celles relatives à l'intervention. Une telle réflexion doit se fonder sur une compréhension et une interprétation partagées des résultats atteints, des changements intervenus (ou non) pour les acteurs majeurs (perception, compétences, attitude, stratégies], des processus d'innovation générés, et de l'évolution du contexte.

Un des facteurs nécessaires pour que le SEPA joue un tel rôle est l'identification des moments clés au cours desquels l'équipe d'intervention se donnera le temps et les moyens de procéder, sur la base des données du suivi, à une lecture critique de ses actions et activités, et pourra réévaluer et valider les résultats (outcomes) et les hypothèses des impacts, les acteurs réellement impliqués et les changements en cours dans leurs pratiques ou attitudes, les évolutions du contexte qu'il s'agit de prendre en compte, etc. Ces moments de réflexivité et d'apprentissage peuvent avoir lieu lors de temps dédiés à l'occasion des ateliers annuels de rendu des résultats et de planification des activités futures, ou pendant de mini-ateliers (ou réunions virtuelles) périodiques dédiés à cette fin. Des moments spécifiques d'évaluation intermédiaires pendant lesquels des évaluateurs internes ou externes réalisent ces évaluations peuvent également être prévus.

Le SEPA peut être intégré à l'intervention avec des moyens humains et financiers spécifiques. Mais il peut aussi être confié à des évaluateurs extérieurs qui viendront collecter l'information pour renseigner les indicateurs et animer les ateliers de réflexion.

Dans tous les cas, il est important de se donner le temps et des ressources suffisantes pour pouvoir collectivement et périodiquement " lever le nez du guidon " de l'action quotidienne et réfléchir de manière critique et stratégique à la question : sommes-nous sur le " bon " chemin de l'impact ? Nos activités contribuent-elles effectivement à nous rapprocher de l'impact désiré ? La forme que prendra le SEPA dépendra in fine de la nature, la taille, et la complexité de l'intervention, et aussi du ou des modes d'intervention privilégiés [voir partie "Étape 1: Quel est le mode d'intervention principal de la recherche ? "). II faut enfin bien sûr veiller à ce que l'investissement attribué au SEPA ne soit pas disproportionné, en temps ou en moyens financiers, et se fasse donc au détriment de la mise en œuvre des activités elles-mêmes. Il n'y a pas de formule standard.

12 Lennie et al., 2011, http://www.betterevaluation.org/sites/default/files/EA_PM\%26E_toolkit_module_2_objectives\%26indicators_for_publication.pdf. 


\section{L'atelier ImpresS ex ante}

Lors de l'application de la démarche ImpresS ex ante, les équipes sont amenées à travailler les questions posées de manière itérative et si possible participative. Construire une intervention en y incluant une réflexion sur l'impact est donc un processus fait de réunions virtuelles et présentielles, d'allers-retours sur les documents, de diagnostics et/ou d'ateliers incluant les chercheurs et les partenaires clés qui montent l'intervention ensemble.

Quelle que soit l'intensité de mise en œuvre de la démarche, l'organisation d'au moins un atelier ex ante présentiel en constitue une étape incontournable. Dans cet atelier se retrouvent idéalement (en fonction des financements disponibles) les représentants des acteurs partenaires, et éventuellement des représentants des acteurs influents et impactés visés par l'intervention.

Le tableau 5 illustre à titre indicatif ce qui peut être inclus dans l'agenda de cet atelier quand les participants sont réunis pendant 1,2 ou 2,5 jours. Il faut bien sûr être flexible quant au timing, et prêt à l'adapter si les participants souhaitent approfondir tel ou tel sujet.

Tableau 5 : Différentes modalités d'atelier participatif ex ante selon le temps disponible.

\begin{tabular}{|c|c|c|c|c|c|}
\hline Avant l'atelier & $\begin{array}{c}\text { Temps } \\
\text { disponible }\end{array}$ & Jour 1 & Jour 2 & Jour 3 & Après l'atelier \\
\hline \multirow[t]{3}{*}{$\begin{array}{l}\text { 1. Diagnostic } \\
\text { initial: } \\
\text { contextes, } \\
\text { acteurs, projets } \\
\text { existants } \\
\text { 2. Premier } \\
\text { brouillon de } \\
\text { récit sur la base } \\
\text { du diagnostic } \\
\text { initial } \\
\text { 3. Envoyer } \\
\text { le récit aux } \\
\text { participants [2 } \\
\text { pages max.) }\end{array}$} & 1 jour & $\begin{array}{l}\text { Introduction, attentes et } \\
\text { présentation du diagnostic ou } \\
\text { brouillon de récit (1h], étape } 1.1 \text { et } \\
\text { vision du futur }\left(30^{\prime} \text { ] }\right. \\
\text { Arbre à problèmes et solutions } \\
\text { [1h30] } \\
\text { Cartographie d'acteurs [1h] } \\
\text { Détermination des outcomes [1h] } \\
\text { Obstacles aux changements et } \\
\text { stratégies [1h] }\end{array}$ & & & $\begin{array}{l}\text { Activités sur la } \\
\text { base des stratégies } \\
\text { Outputs sur la base } \\
\text { des activités et } \\
\text { outcomes } \\
\text { Chemins de } \\
\text { l'impact } \\
\text { Finalisation du } \\
\text { récit } \\
\text { Suivi-évaluation et } \\
\text { apprentissage } \\
\text { Document final }\end{array}$ \\
\hline & 2 jours & $\begin{array}{l}\text { Introduction, attentes et } \\
\text { présentation du diagnostic ou } \\
\text { brouillon de récit [1h] } \\
\text { Vision du futur }\left(30^{\prime}\right] \\
\text { Arbre à problèmes et solutions (2h] } \\
\text { Cartographie d'acteurs [1h] } \\
\text { Détermination des outcomes (1h] } \\
\text { Obstacles aux changements [1h] }\end{array}$ & $\begin{array}{l}\text { Stratégies pour } \\
\text { surmonter les obstacles } \\
\text { [1h] } \\
\text { Activités sur la base des } \\
\text { stratégies [1h30] } \\
\text { Outputs des activités [1h] } \\
\text { Chemin de l'impact \& } \\
\text { chemins alternatifs [1h30] } \\
\left.\text { Reprise du récit [ } 30^{\prime}\right]\end{array}$ & & $\begin{array}{l}\text { Finalisation du } \\
\text { récit } \\
\text { Suivi-évaluation et } \\
\text { apprentissage } \\
\text { Document final }\end{array}$ \\
\hline & 2,5 jours & $\begin{array}{l}\text { Introduction, attentes et } \\
\text { présentation du diagnostic ou } \\
\text { brouillon de récit [1h] } \\
\left.\text { Vision du futur [ } 30^{\prime}\right] \\
\text { Arbre à problèmes et solutions [2h] } \\
\text { Cartographie d'acteurs [1h] } \\
\text { Détermination des outcomes [1h] } \\
\text { Obstacles aux changements [1h] }\end{array}$ & $\begin{array}{l}\text { Stratégies pour } \\
\text { surmonter les obstacles } \\
\text { (1h] } \\
\text { Activités sur la base des } \\
\text { stratégies [2h] } \\
\text { Outputs des activités } \\
\text { (1h30) } \\
\text { Chemin de l'impact \& } \\
\text { chemins alternatifs (1h30) }\end{array}$ & $\begin{array}{l}\text { Finalisation du } \\
\text { récit (1h30] } \\
\text { Suivi- } \\
\text { évaluation } \\
\text { participatif et } \\
\text { apprentissage } \\
\text { (2h) }\end{array}$ & Document final \\
\hline
\end{tabular}


Si l'atelier ne dure qu'un jour, on se focalise sur l'arbre à problèmes et solutions, les acteurs et les résultats qu'on souhaite provoquer. Si le groupe est actif et les discussions sont conclusives, il est possible de travailler également les obstacles et les stratégies pour les surmonter. Après l'atelier, il faudra identifier les activités ressortant de ces stratégies et les outputs, construire le chemin de l'impact et imaginer des scénarios alternatifs, finaliser le récit et éventuellement identifier les indicateurs d'outcome à suivre.

$S^{\prime}$ il est possible de rajouter une deuxième journée au programme, alors les obstacles et stratégies pour les surmonter seront travaillés plus en détail, et les activités à mettre en place en fonction des stratégies identifiées, et par conséquent les produits de ces activités, seront définis. II y aura aussi le temps de visualiser le chemin de l'impact et d'imaginer des scénarios alternatifs. Éventuellement, le récit sera brièvement travaillé en consolidant tous les éléments obtenus.

Finalement, s'il est possible de travailler une demi-journée supplémentaire avec les participants, le système de suivi-évaluation participatif sur la base des indicateurs d'outcome peut être identifié. Dans ce cas, le récit peut être finalisé, ou alors plus de temps sera pris dans les journées antérieures pour aller dans le détail lors de certaines sessions.

À noter que l'existence d'un diagnostic plus ou moins détaillé est un préalable essentiel pour le succès de I'atelier. À défaut, un travail spécifique devra être entrepris pour ce faire en tout début de l'atelier, avec les acteurs participant, ce qui permettra de construire une vision globale du chemin de l'impact.

\section{Trucs \& Astuces $\mathrm{n}^{\circ} 6$ : Animation de l'atelier}

1. Pour la cartographie d'acteurs, vous pouvez imaginer un jeu de rôle : chaque participant représente un type d'acteurs $[O P, O N G$, ministère, institut de recherche, etc.). Chaque participant répond à différentes questions:

- Avec quels autres acteurs cet acteur est-il en lien dans la situation actuelle ? Quel type de relation les relie?

- Quel impact aura la solution / innovation proposée par l'intervention sur cet acteur ? Est-ce un impact négatif ou positif ?

- Cet acteur est-il favorable ou non à la solution / innovation proposée par l'intervention ? Son influence (positive ou négative) par rapport à la solution proposée est-elle faible, intermédiaire ou forte?

2. Par rapport aux résultats [outcomes] :

- Quels sont les changements qui vous semblent nécessaires pour arriver à l'impact ? Quels sont les obstacles?

- Comment se comporterait chaque acteur par rapport aux changements des pratiques et comportements que l'intervention souhaite provoquer chez l'acteur ? Y serait-il favorable ou au contraire y opposerait-il une résistance?
3. Si votre public est actif et qu'il participe facilement, vous pouvez utiliser l'interface ImpresS pour générer l'arbre à problèmes et solutions, la cartographie d'acteurs, le graphe d'outcomes et le chemin de l'impact. Si votre public est moins actif, il est recommandé de faire les exercices en utilisant des post-it ou des flip charts.

4. Si vous vous divisez en groupes pour travailler certaines sections, par exemple les outcomes ou les stratégies pour surmonter les obstacles, vous pouvez aussi appliquer un World Café. C'est un outil adapté pour que tout le monde puisse contribuer à l'analyse de plusieurs éléments. Par exemple, quand les outcomes et les changements identifiés sont nombreux, il est plus efficace d'y travailler par petits groupes (pas plus de 3 personnes], mais pour que tous les participants les discutent tous, les groupes y travailleront à tour de rôle sur des périodes de 30 minutes. Chaque groupe discute et complète le travail fait par le groupe antérieur. L'exercice se termine par une session de synthèse en plénière. Vous pouvez vous référer au lien suivant : http://www.pratiques-collaboratives.net/WorldCafe-une-presentation-du-comment-faire.html 


\section{L'interface ImpresS}

Le chantier ImpresS s'est doté d'un système d'information pour capitaliser les résultats des études de cas analysées dans le cadre de la démarche ex post et pour faciliter la visualisation et la systématisation des chemins de l'impact issus de l'application de la démarche ex ante. Un informaticien, sous la conduite de l'équipe ImpresS et des chercheurs de l'unité de recherche AIDA, a développé une interface de saisie et de dialogue en ligne, appelée « interface ImpresS » (figure 11).

Les chercheurs du Cirad peuvent se connecter à l'interface avec leur identifiant Cirad en utilisant le lien : https://intranet-impress.cirad.fr/index.php

Pour aller directement sur la création d'un nouveau scénario ex ante, il faut suivre ce lien :

https://intranet-impress.cirad.fr/index.php/scenario/nouveau_scenario

L'interface permet aux équipes de visualiser de manière flexible et adaptable certains éléments clés utiles pour la construction participative. Les outils fournis par l'interface peuvent être adaptés pour l'exercice miroir (voir Trucs $\&$ astuces $n^{\circ} 5$ ), via un dialogue entre équipes projets et l'équipe ImpresS. Au final, il contribue à générer une mémoire institutionnelle et une systématisation des connaissances sur l'évaluation de l'impact au Cirad.

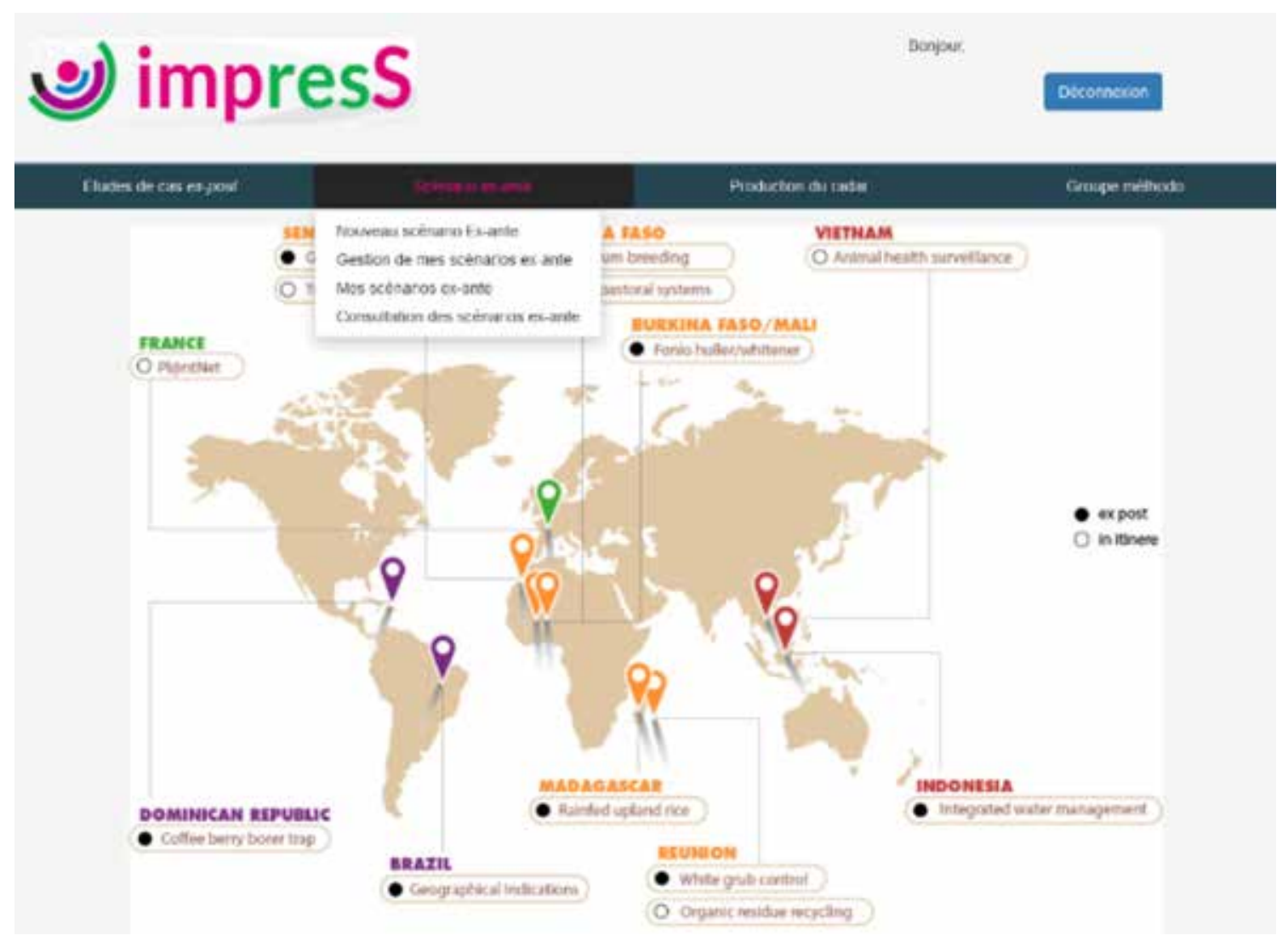

Figure 11: Création d'un nouveau scénario ex ante dans l'interface ImpresS.

La saisie des données permettant de visualiser les outils peut être effectuée à l'aide d'un mode " déconnecté ". Cette fonction permet de conserver les données de la saisie en cours du scénario ex ante lors de coupures de courant intempestives ou de fermetures inopinées du navigateur internet. Une synchronisation de l'interface de saisie sur le serveur ImpresS permet la mise à jour et le stockage sur internet. 
Parmi les opérations autorisées [création / suppression d'un scénario ex ante), il est possible de dupliquer un scénario existant afin de permettre la construction d'un nouveau scénario à partir de celui-ci. Tous les scénarios ex ante sont consultables par leur auteur exclusivement et les représentations graphiques créées sont téléchargeables.

L'interface permet de stocker des informations et de visualiser les outils graphiques suivants:

1. Carte d'identité : cet outil permet de stocker des informations basiques issues de la réflexion ex ante, incluant la possibilité de sauvegarder le récit sous format Word (figure 12).

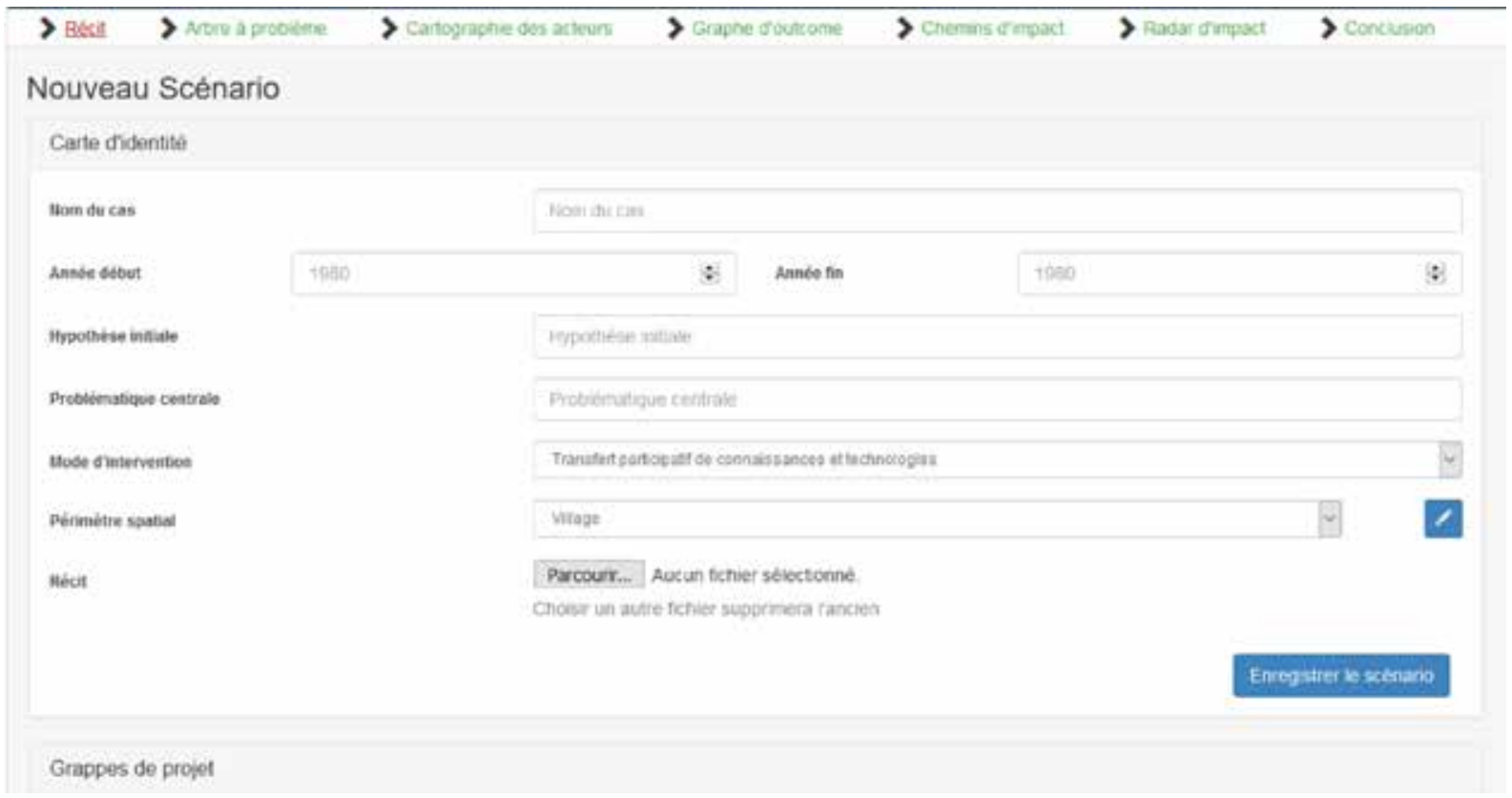

Figure 12 : Carte d'identité du scénario ex ante dans l'interface ImpresS.

2. Arbre à problèmes et solutions : cet outil permet de représenter l'arbre à problèmes ou l'arbre à solutions, ou les deux sur le même graphe (figure 13).

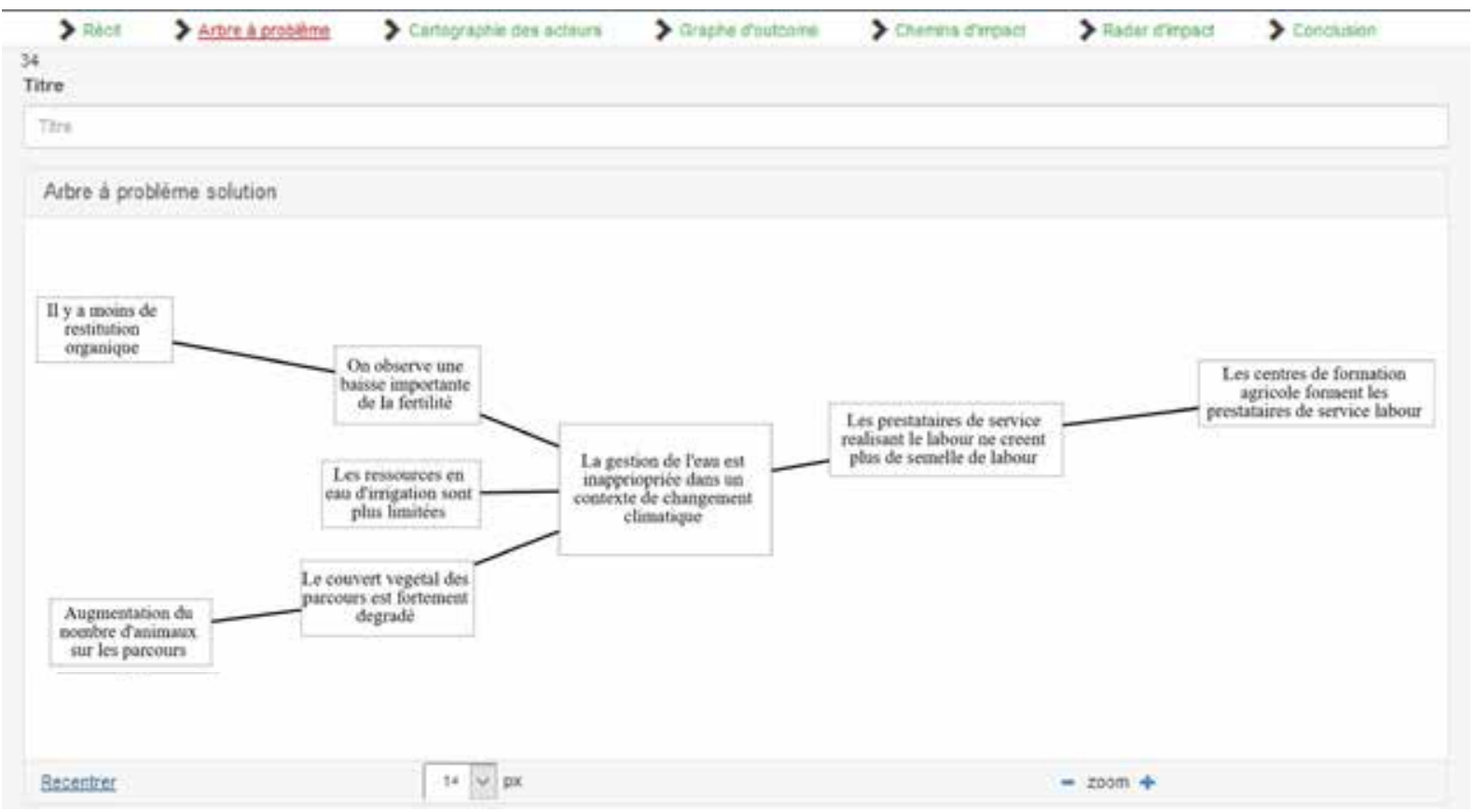

Figure 13 : Représentation d'un arbre à problèmes dans l'interface ImpresS. 
3. Cartographie des acteurs : cet outil permet de représenter le rôle des acteurs, les relations entre eux et de caractériser les types de relations (figure 14].

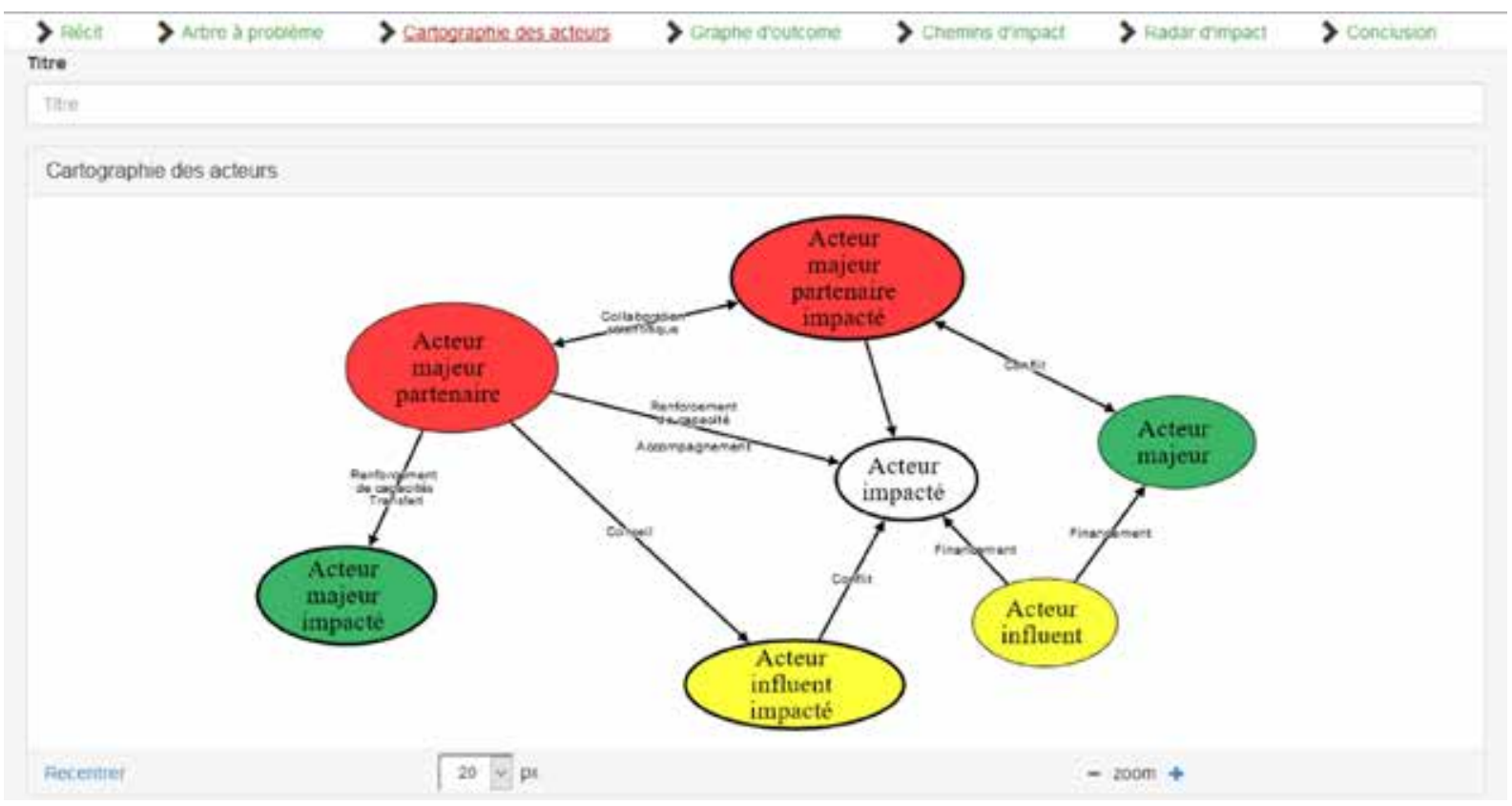

Figure 14 : Cartographie des acteurs dans l'interface ImpresS.

4. Graphe d'outcomes : cet outil permet de visualiser les impacts, outcomes, changements majeurs, obstacles, stratégies et activités dans un seul graphe [figure 15].

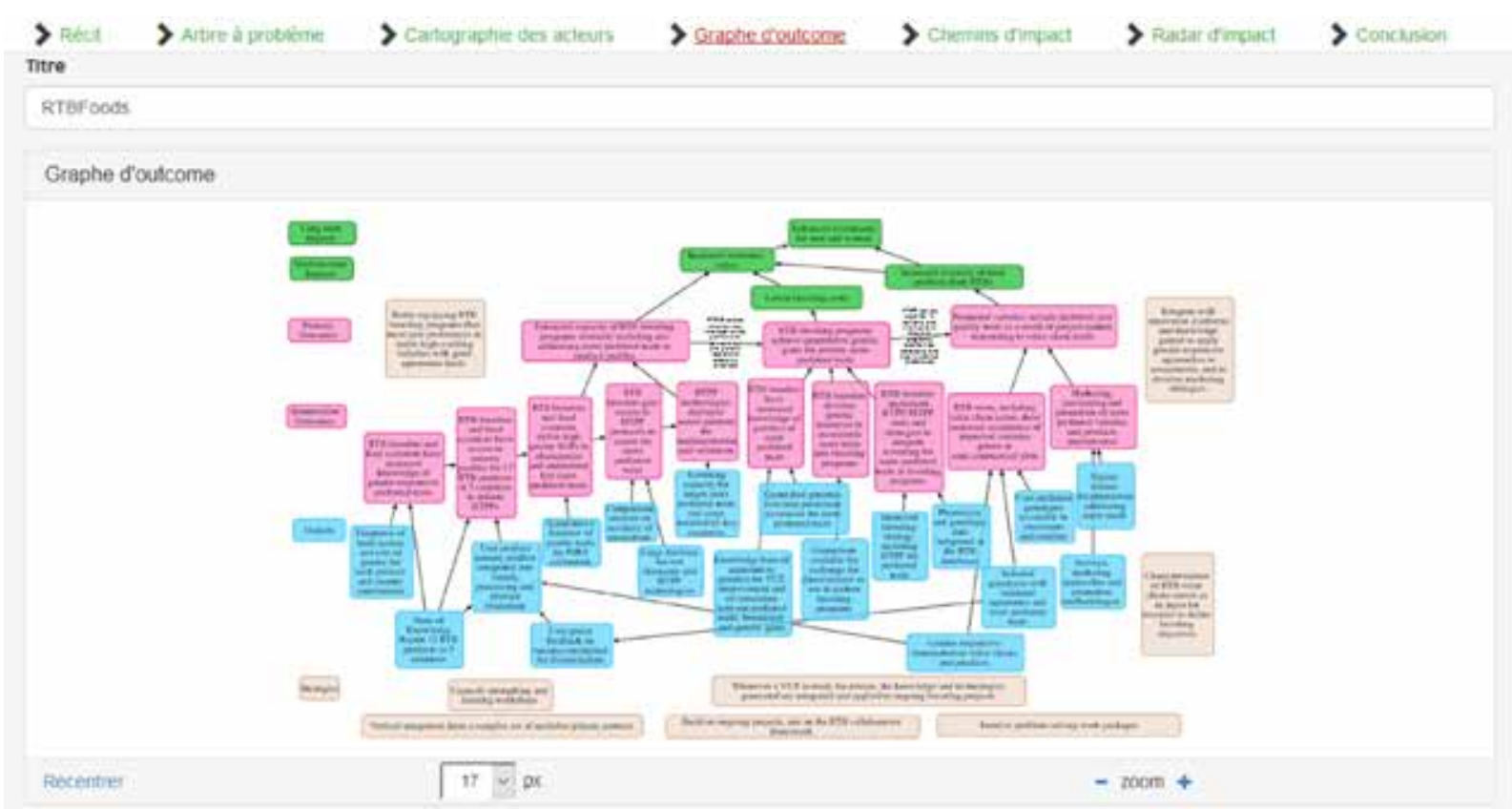

Figure 15 : Exemple de graphe d'outcomes du projet RTB Foods dans l'interface ImpresS. 
5. Chemins de l'impact : cet outil permet de visualiser le chemin de l'impact et le dupliquer pour pouvoir construire deux chemins de l'impact alternatifs [figure 16].

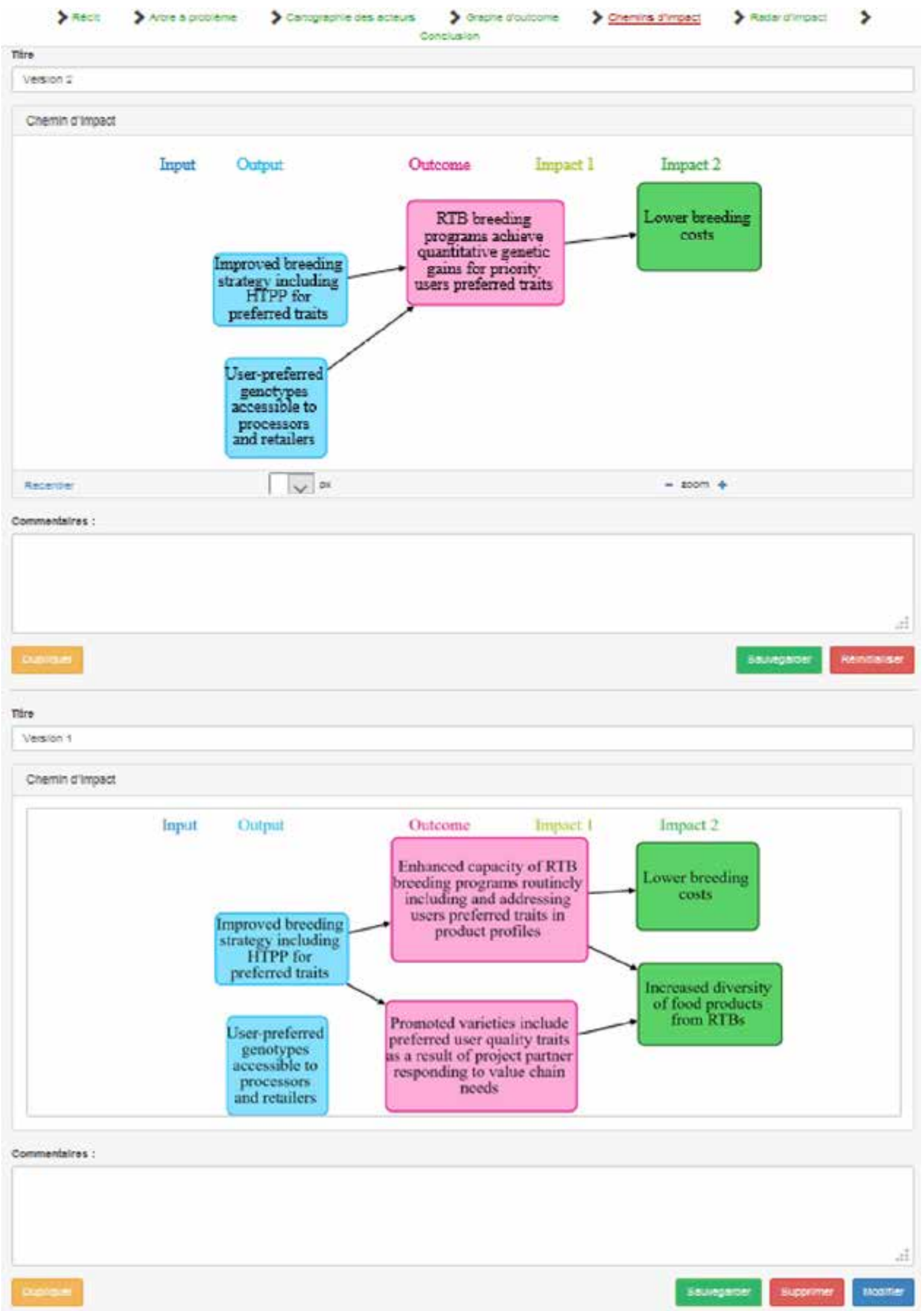

Figure 16 : Chemins de l'impact alternatifs dans l'interface ImpresS. 
6. Le radar des impacts : cet outil permet la visualisation des domaines d'impacts, incluant des hypothèses d'ampleur et d'intensité des impacts (figure 17).

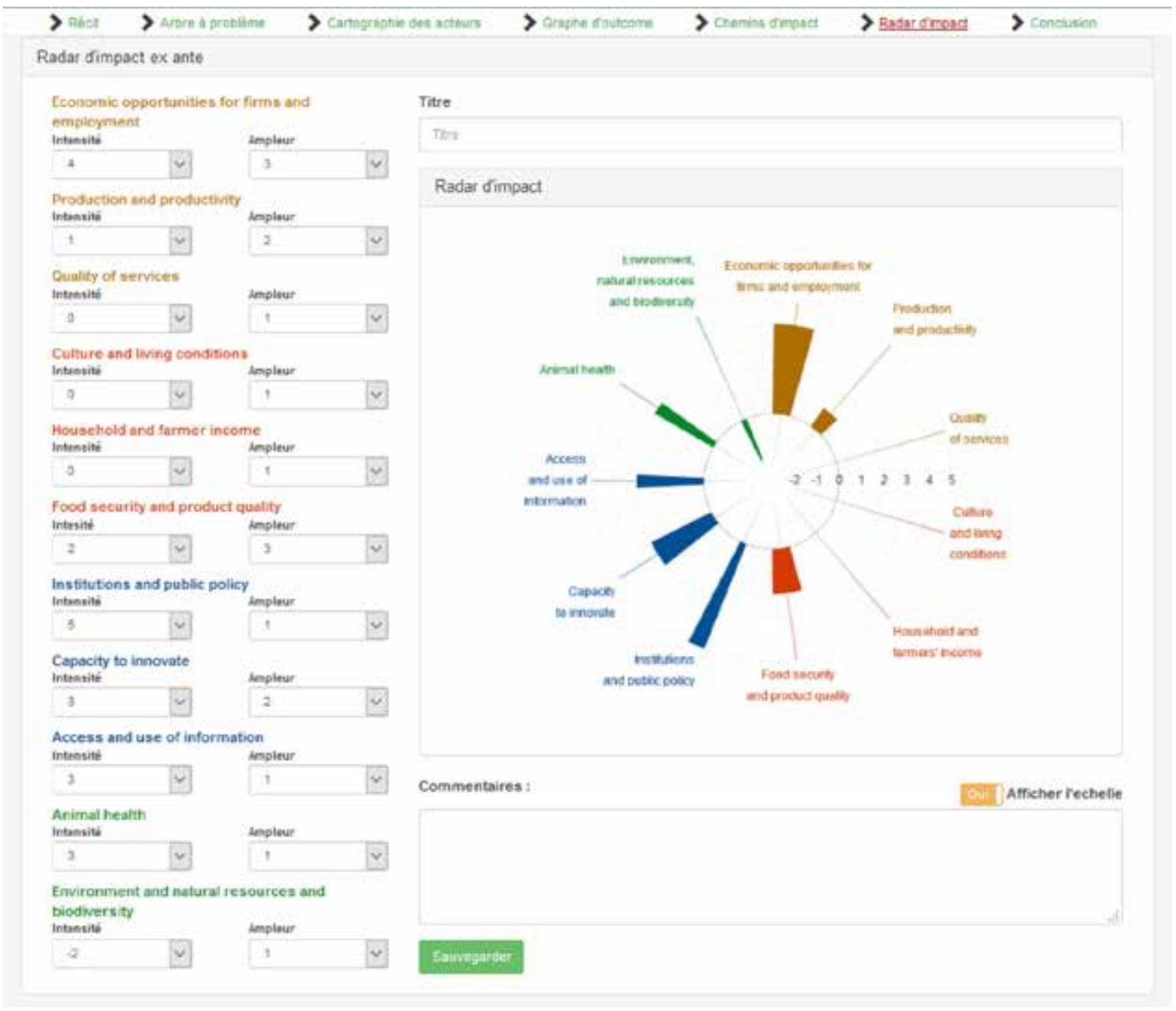

Figure 17 : Un radar des impacts dans l'interface ImpresS. 



\section{Adapter la démarche ImpresS ex ante à différentes situations}

\section{Adapter la démarche à différentes ressources disponibles}

La démarche ImpresS ex ante est adaptable à différentes exigences de délais, de ressources et de capacités. Mais, même si le temps n'est pas une contrainte, il faut éviter d'en passer trop à développer des chemins de l'impact hypothétiques trop détaillés ; il faut trouver le juste équilibre entre un récit trop simpliste et un récit trop détaillé. La réflexion doit surtout aider à concevoir et planifier des interventions plus plausibles et efficaces, en se posant des questions stratégiques sur la meilleure manière de s'y prendre, en maximisant la probabilité que les produits de l'intervention soient transformés en résultats appropriés par les acteurs. Dans cette construction, la participation des acteurs et le travail en boucle sont clés car ils permettent d'enrichir le chemin de l'impact hypothétique, de le rendre progressivement plus réaliste, plus crédible, et favorisent l'apprentissage et l'appropriation collective de la vision de l'intervention qui émerge de ces réflexions.

Au final, la démarche proposée n'est pas figée : elle propose certes des étapes cohérentes et certains outils, mais elle laisse aux équipes le choix des méthodes qu'elles considèrent les plus adaptées pour répondre aux questions mises en avant dans la démarche. Obtenir des réponses pertinentes à ces questions est plus stratégique qu'utiliser tel ou tel outil. De ce fait, les chercheurs en phase de construction d'une intervention sont encouragés à ne pas mettre en œuvre ImpresS ex ante tout seuls : ils sont invités dans la mesure du possible à s'appuyer sur et à s'intégrer dans des équipes multidisciplinaires et multipartenaires, sur l'équipe ImpresS et les chargés de valorisation du Cirad.

La démarche proposée est adaptable aux ressources en temps, en budget, et en capacités mobilisables pour mener une réflexion sur l'impact ex ante d'une intervention. Si les ressources disponibles lors de la phase de montage d'une intervention ne permettent pas une participation adéquate des partenaires futurs de l'intervention aux réflexions ex ante, l'équipe en charge de ce montage pourra toujours mettre à profit toutes les étapes et composantes de la réflexion. Mais dans ce cas, il est fortement recommandé d'intégrer à la proposition une demande de financement spécifique permettant d'organiser un atelier multi-acteurs en tout début d'intervention, dont le but sera de partager, améliorer et valider entre les partenaires et/ou les acteurs majeurs de l'intervention le chemin de l'impact ex ante imaginé par les chercheurs. Certains bailleurs peuvent être très sensibles à la pertinence d'une telle demande, car elle démontre que les chercheurs comprennent véritablement la nature participative et multi-acteurs des processus d'innovation, dont dépend l'obtention de l'impact.

La figure 18 (page suivante) montre différentes options de développement de la démarche selon les ressources disponibles avant ou après l'obtention du financement. 


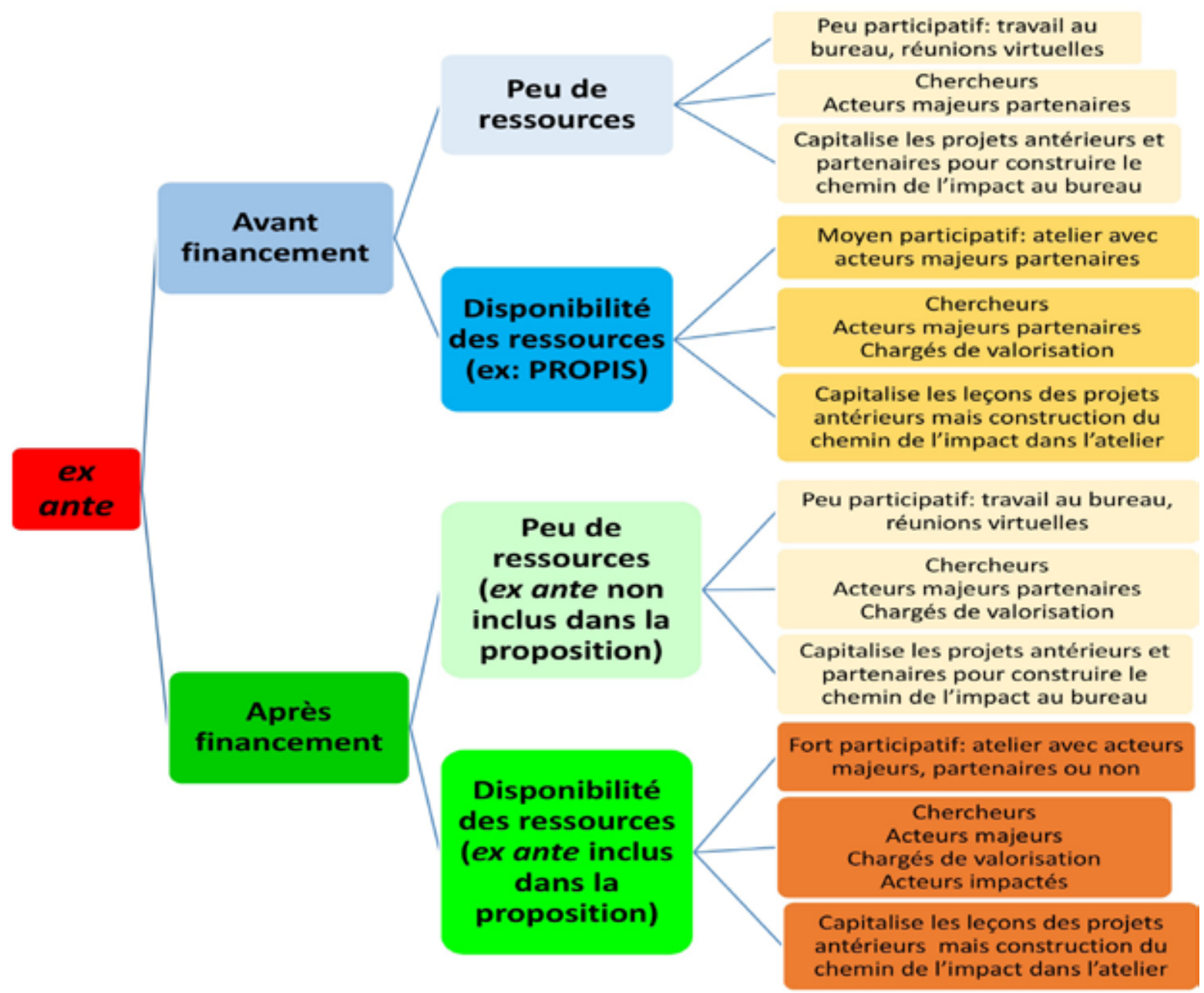

Figure 18 : Adaptabilité de la démarche ImpresS ex ante. 


\section{Adapter le récit pour différentes audiences}

Dans la démarche proposée dans ce document, l'équipe en charge de la réflexion ex ante produit un seul récit. Cependant, le récit a vocation à faciliter la communication avec différentes audiences, chacune ayant ses attentes propres : il s'agit donc de pouvoir, sans en trahir le fond, moduler le récit pour être en position de dialogue avec les différentes audiences.

Les partenaires de l'intervention sont les premiers concernés par le récit, il faut donc s'assurer que celui-ci saura les convaincre de la pertinence et de la plausibilité de l'intervention proposée et du chemin de l'impact imaginé, et parviendra à les motiver à participer activement.

La communauté scientifique, dont les chercheurs, forme une autre audience que le récit doit savoir convaincre : il est important dans ce cas de mettre en avant les étapes initiales du chemin de l'impact, qui sont celles dans lesquelles la recherche a souvent un rôle fondamental, dont la genèse des produits.

Les bailleurs sont bien entendu l'une des audiences clés pour le récit ex ante : il s'agit de les convaincre "d'acheter le récit », en s'assurant dans la mesure du possible que le récit produit résonne avec leurs propres préoccupations, attentes, priorités et visions de l'impact, et leurs propres cadres de pensée (par exemple l'attente par de nombreux bailleurs de la présentation d'un cadre logique que la démarche ImpresS ex ante peut très bien contribuer à développer - voir encadré 7, page suivante). Mais cela doit être fait sans masquer la complexité des interventions proposées, la nécessité de flexibilité de la programmation au cours du temps pour s'adapter aux aléas, ni passer sous silence le pas de temps pertinent pour obtenir l'impact souhaité. Le récit peut aussi être modulé en fonction du fait que certains bailleurs ne se contentent pas de financer des interventions, mais en sont aussi des partenaires dont le rôle doit donc être rendu visible à leur intention.

Les chargés de valorisation du Cirad sont particulièrement bien positionnés pour articuler la mise en œuvre de la démarche ImpresS ex ante avec les attentes des bailleurs. Les actions pour promouvoir la culture de l'impact passent donc par la collaboration de l'équipe ImpresS avec les chargés de valorisation, par des formations pour aider ces derniers à s'approprier la démarche ex ante (voir annexe) mais aussi à trouver des solutions qui l'améliorent, et enfin par un appui méthodologique à tous ceux qui montent des projets. 


\section{Encadré 7 : Exemple d'éléments issus de la démarche Impress permettant de mieux renseigner les demandes de bailleurs}

La démarche ImpresS ex ante permet de réfléchir à différents éléments demandés dans certains appels d'offres et à propositions, ou de remplir un cadre logique, etc. Ci-dessous des exemples des différents formats remplis en mettant à profit la démarche ImpresS.

\section{BIOPHORA : Cadre logique rempli après une réflexion menée selon la démarche ImpresS ex ante [LEAPACRI]}

\begin{tabular}{|c|c|}
\hline Research outputs & Indicators \\
\hline $\begin{array}{l}\text { Gene bank for native } \\
\text { entomopathogens }\end{array}$ & $\begin{array}{l}\text { Candidate strains }(>3) \\
\text { Patent }(>1)\end{array}$ \\
\hline $\begin{array}{l}\text { Optimal phoretic agent- } \\
\text { entomopathogen } \\
\text { system }\end{array}$ & Candidate combinations \\
\hline $\begin{array}{l}\text { SIT-based biocontrol } \\
\text { technologies for fruit } \\
\text { flies and mango } \\
\text { anthracnose }\end{array}$ & Patent $(>1)$ \\
\hline $\begin{array}{l}\text { Auto-dissemination } \\
\text { stations ready-for-use }\end{array}$ & $\begin{array}{l}\text { Commercial availability } \\
\text { of station (1) }\end{array}$ \\
\hline Academic publications & $\begin{array}{l}(>4) \text { scientific papers in } \\
\text { peer-reviewed journals }\end{array}$ \\
\hline Implementation strategy & Technical guidelines \\
\hline Risk monitoring plan & $\begin{array}{l}\text { Monitoring plan for } \\
\text { regulatory agency }\end{array}$ \\
\hline $\begin{array}{l}\text { Technical guidelines, } \\
\text { communication and field } \\
\text { demonstration }\end{array}$ & $\begin{array}{l}\mathrm{Nb} \text { of field } \\
\text { demonstrations }(>2) \\
\mathrm{Nb} \text { of attendees }(>100)\end{array}$ \\
\hline $\begin{array}{l}\text { Stakeholder platform for } \\
\text { biocontrol innovations }\end{array}$ & $\mathrm{Nb}$ of annual meetings \\
\hline $\begin{array}{l}\text { Training workshops } \\
\text { for producers, } \\
\text { governmental bodies, } \\
\text { pesticide retailers, etc. }\end{array}$ & $\begin{array}{l}(>4) \text { training and } \\
\text { educational operations } \\
\mathrm{Nb} \text { of attendees }(>100)\end{array}$ \\
\hline $\begin{array}{l}\text { Training for graduate } \\
\text { students }\end{array}$ & $\begin{array}{l}\mathrm{Nb} \text { of graduate students } \\
\text { trained }\end{array}$ \\
\hline Policy briefs & $\mathrm{Nb}$ of policy briefs (3) \\
\hline
\end{tabular}

\begin{tabular}{|l|l|}
\hline \multicolumn{1}{|c|}{ Outcomes } & \multicolumn{1}{|c|}{ Indicators } \\
\hline $\begin{array}{l}\text { Improved pest } \\
\text { management practices } \\
\text { (better knowledge of IPM } \\
\text { and innovative } \\
\text { technologies) }\end{array}$ & $\begin{array}{l}\text { Quantity of insecticide use } \\
\text { Nb of farmers using } \\
\text { developed technologies } \\
\text { and IPM strategies }\end{array}$ \\
\hline $\begin{array}{l}\text { Organizational capacity of } \\
\text { mango producers (for } \\
\text { better access to and use of } \\
\text { biopesticides and collective } \\
\text { pest management) }\end{array}$ & $\begin{array}{l}\text { Nb and size of farmer } \\
\text { organizations engaged in } \\
\text { pest biocontrol (good } \\
\text { practices) }\end{array}$ \\
\hline $\begin{array}{l}\text { Emergence of local private } \\
\text { units dedicated to } \\
\text { biopesticide production and } \\
\text { commercialization } \\
\text { (valorisation of indigenous } \\
\text { biocontrol agents) }\end{array}$ & $\begin{array}{l}\text { Nb of partnerships } \\
\text { between local } \\
\text { entrepreneurs and Biobest } \\
\text { Nb of local entrepreneurs } \\
\text { Conventional phytosanitary } \\
\text { industry shift to biocontrol }\end{array}$ \\
\hline $\begin{array}{l}\text { Emergence of a new } \\
\text { regulatory framework for } \\
\text { SIT and biopesticides }\end{array}$ & $\begin{array}{l}\text { New national regulation } \\
\text { and support framework } \\
\text { Sustained risk monitoring }\end{array}$ \\
\hline $\begin{array}{l}\text { Governmental bodies and } \\
\text { private sector strengthened } \\
\text { for innovation outscaling }\end{array}$ & $\begin{array}{l}\text { Nb of field schools } \\
\text { products }\end{array}$ \\
\hline $\begin{array}{l}\text { Evidence-based policy } \\
\text { making }\end{array}$ & $\begin{array}{l}\text { New policy supporting pest } \\
\text { biocontrol }\end{array}$ \\
\hline
\end{tabular}

\begin{tabular}{|l|}
\hline \multicolumn{1}{|c|}{ Impact } \\
\hline Economic impact \\
Increase of fruit production \\
and quality (residues) and \\
organoleptic quality of \\
mangoes \\
Increased income along the \\
chain value \\
Enhanced resilience of mango \\
production systems \\
New market opportunities for \\
mango production \\
(conventional, \\
organic/certified/export, \\
processing) \\
\hline $\begin{array}{l}\text { Social impact } \\
\text { Improved food and nutrition } \\
\text { security, better health } \\
\text { Employment opportunities } \\
\text { Organizational capital and } \\
\text { capacities developed } \\
\text { Women and youth benefit } \\
\text { from new income generating } \\
\text { activities including } \\
\text { transformation }\end{array}$ \\
\hline Environmental impact \\
Reduction of pesticide use \\
Benefits for functional \\
biodiversity in ecosystems \\
\hline
\end{tabular}

2. Gender-SMART : Exemple d'un chemin de l'impact global articulé avec les Work Packages [H2020]

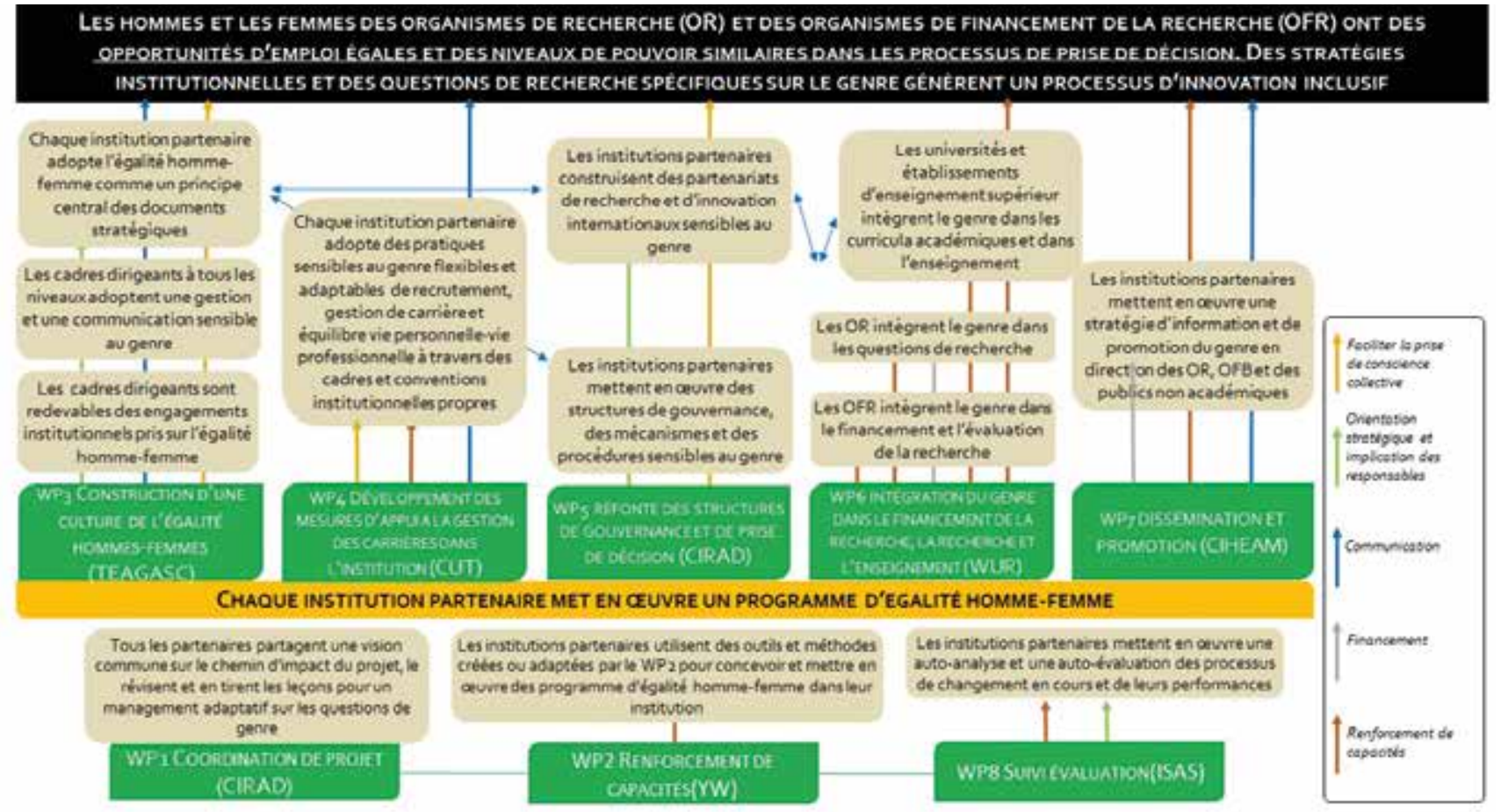




\section{Conclusion}

La démarche ImpresS ex ante vise à rendre explicites les mécanismes par lesquels on souhaite qu'une action de recherche contribue à générer des résultats, et les stratégies qu'on se donne pour y parvenir. Les mécanismes relèvent souvent de l'implicite, de l'impensé ou de l'imprévu dans la conception et la mise en œuvre conventionnelles de la recherche, et la démarche est donc exigeante ; elle guide l'élaboration collective - et si possible participative - de " chemins de l'impact hypothétiques et plausibles ", prenant en compte les acteurs majeurs et les changements souhaités, les obstacles et des scénarios alternatifs permettant d'y faire face, le renforcement des capacités ou l'interaction avec les décideurs politiques. Le but ultime est d'aider les équipes de recherche et leurs partenaires à structurer intelligemment la conception, la mise en œuvre, le pilotage et le suivi des interventions qu'ils entreprennent pour maximiser les chances d'impacter.

Il n'est jamais simple de mettre en œuvre une approche pleinement participative pour concevoir et programmer une action de recherche. La démarche ImpresS considère cependant que cette participation est hautement souhaitable, soit avant la conception de l'intervention, soit juste avant son démarrage. La participation des acteurs partenaires de l'intervention dans la réflexion ex ante renforce la plausibilité et la faisabilité des chemins de l'impact proposés. Elle permet aussi de construire une vision réellement partagée des objectifs d'une intervention et facilite la mobilisation au cours de la mise en œuvre de cette dernière.

S'il n'est pas nécessaire que tous les chercheurs et partenaires maîtrisent la méthode dans son ensemble, il est toutefois important d'avoir en tête quelques aspects stratégiques : acteurs, périmètre, obstacles, stratégies, chemin de l'impact, ainsi que la capacité à utiliser une panoplie d'outils, parfois assez simples, permettant de traiter ces aspects. La démarche ImpresS n'est ni une recette à appliquer, ni un cadre imposé, mais une approche pour structurer la réflexion collective, améliorer la réflexivité et développer une culture de l'impact au sein du Cirad et chez ses partenaires.

Bien menée, la démarche aboutit à un récit plausible explicitant comment une intervention contribue aux impacts. Ce récit est la distillation de divers ingrédients dont l'agencement vise l'adhésion, voire l'émotion, des partenaires et des bailleurs de fonds, mais sans entrer dans la promesse des impacts. On formule des hypothèses de façon rigoureuse, en les explicitant de façon transparente et honnête. Ce récit peut être adapté dans sa forme pour s'adresser aux bailleurs, aux acteurs, ou aux partenaires, mais ses différentes versions doivent rester absolument cohérentes.

L'équipe ImpresS espère que cette démarche rendra les équipes plus performantes dans la conception de leurs interventions. Elle suivra sa mise en œuvre dans différents projets et tirera profit de tous les feedbacks reçus de la part des utilisateurs pour l'affiner et l'améliorer. 


\section{Liste des figures, tableaux, encadrés et trucs \& astuces}

\section{Figures}

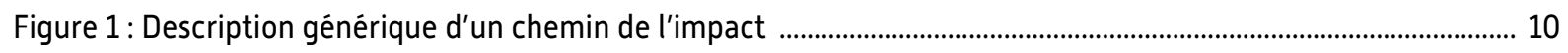

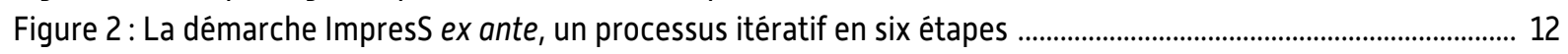

Figure 3 : Exemples d'arbre à problèmes (a) et d'arbre à solutions (b) (cas du projet GITES) .............................................17

Figure 4 : Une partie de la grappe des projets identifiée par le projet GITES ................................................................... 19

Figure 5 : Exemple de cartographie d'acteurs adaptée du projet GITES ........................................................................ 21

Figure 6 : Trois chemins de l'impact globaux interconnectés qui montrent la contribution de la recherche

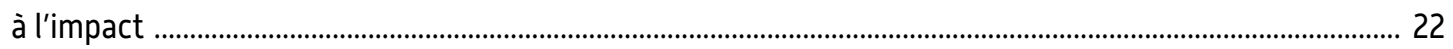

Figure 7 : Archétypes des situations d'intervention de la recherche au Cirad ................................................................. 23

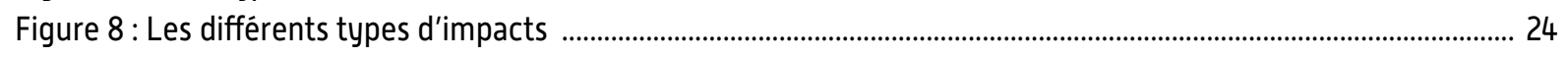

Figure 9 : Radar des impacts pour l'étude de cas « Sélection participative du sorgho au Burkina Faso » ................... 25

Figure 10 : Visualisation schématique du chemin de l'impact avec mise en évidence des situations

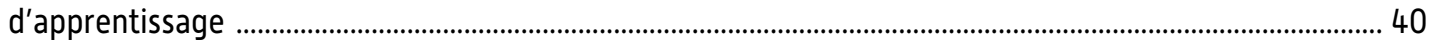

Figure 11 : Création d'un nouveau scénario ex ante dans l'interface ImpresS .................................................................47

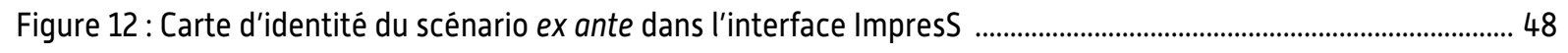

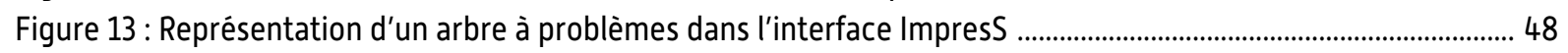

Figure 14 : Cartographie des acteurs dans l'interface ImpresS ........................................................................................49

Figure 15 : Exemple de graphe d'outcomes du projet RTB Foods dans l'interface ImpresS ..............................................49

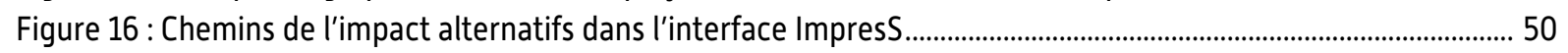

Figure 17 : Un radar des impacts dans l'interface ImpresS .................................................................................................. 51

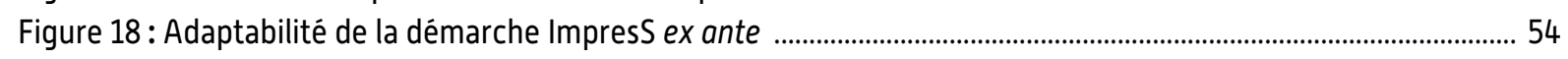

Figure 19 : Articulations entre démarche projet et démarche Impres S ex ante ............................................................. 62

\section{Tableaux}

Tableau 1 : Les 11 domaines d'impacts identifiés dans le cadre de la démarche ImpresS .............................................. 26

Tableau 2 : Exemple de la déclinaison de stratégies « à grain fin » en activités et en produits (projet Maggi

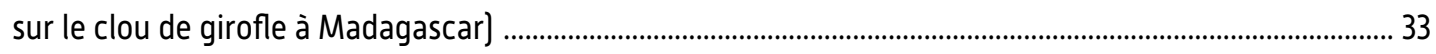

Tableau 3 : Exemple de la déclinaison de stratégies « à gros grain » en activités et en produits (projet

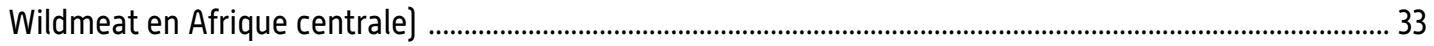

Tableau 4 : Types de capacités définies dans les études de cas ImpresS .........................................................................38

Tableau 5 : Différentes modalités d'atelier participatif ex ante selon le temps disponible ............................................. 45

\section{Encadrés}

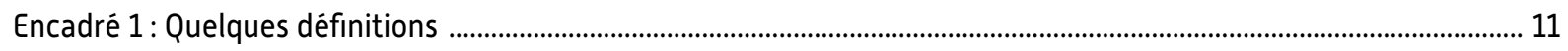

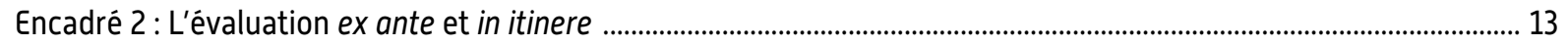

Encadré 3 : Quelques exemples d'hypothèses des impacts tirés d'expériences d'analyses ex ante ............................... 16

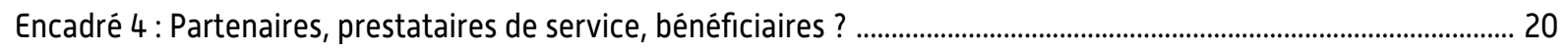

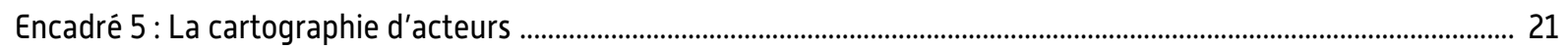

Encadré 6 : Réfléchir les changements souhaitables, les obstacles qui s'y opposent et les stratégies pour surmonter ces derniers ................................................................................................................................... 32

Encadré 7 : Exemple d'éléments issus de la démarche ImpresS permettant de mieux renseigner les demandes

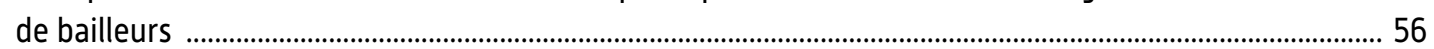

\section{Trucs $\&$ astuces}

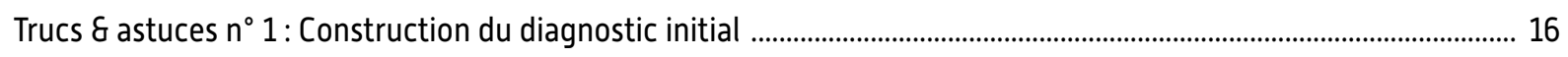

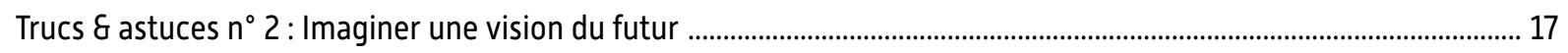

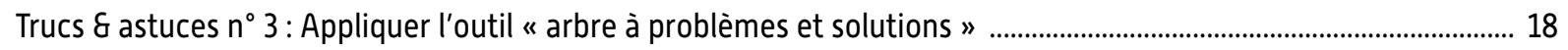

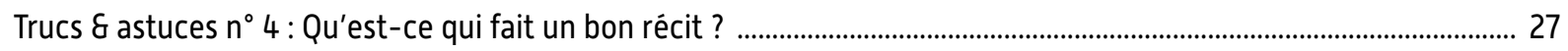

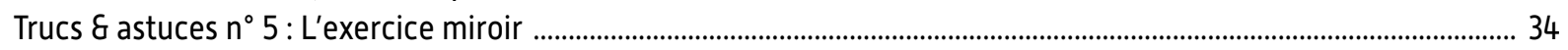

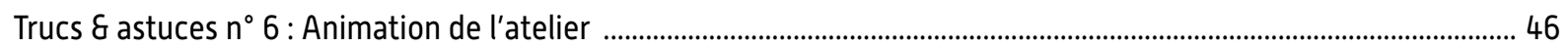




\section{Glossaire}

Acteurs : Individus et organisations jouant un rôle dans le processus d'innovation étudié. On distingue quatre catégories d'acteurs, non mutuellement exclusives, en fonction de la participation de ces derniers dans le processus d'innovation:

- acteurs majeurs partenaires de l'intervention avec lesquels une interaction directe est souhaitée ;

- acteurs majeurs clés dans le processus d'innovation mais qui ne sont pas partenaires de l'intervention ;

- acteurs influents susceptibles d'influencer positivement ou négativement le processus d'innovation sans avoir un rôle d'acteurs actifs dans le processus d'innovation ;

- acteurs impactés qui sont positivement ou négativement impactés par le processus d'innovation.

Si la délimitation entre ces quatre catégories s'avère floue, l'équipe projet fera un choix qui sera explicité.

Apprentissage : Processus d'acquisition de savoirs, savoir-faire et savoir-être d'un individu ou d'un collectif par l'observation, l'échange entre individus et la mise en pratique.

Capacité : Aptitude à exercer des fonctions, résoudre des problèmes, fixer et atteindre des objectifs.

Chemin de l'impact: Description d'un processus d'innovation qui met en évidence des relations de causalité entre les moyens (inputs] mobilisés par une intervention, les produits [outputs) de l'intervention, les résultats [outcomes], qui se matérialisent directement au niveau des utilisateurs des produits [outputs] de l'intervention, et les impacts de $1^{\text {er }}$ et $2^{\mathrm{e}}$ niveaux.

Changement d'échelle : Extension géographique ou du nombre d'adoptants d'une innovation [scaling out] et accroissement du nombre de types d'acteurs et des arrangements entre acteurs liés au déploiement d'une innovation [scaling up]. Le changement d'échelle implique une transformation des connaissances et des techniques à travers les réseaux des acteurs impliqués dans le changement d'échelle, et l'extension de processus d'apprentissage. L'échelle peut être locale [village, canton, etc.], régionale (infranationale), nationale, régionale (supranationale dans des espaces contigus ou pas) ou globale [supranationale dans des espaces non contigus].

Chronogramme : Il permet de visualiser le récit de l'innovation dans sa dimension temporelle en précisant les évènements marquants.

Évaluation ex post / ex ante / in itinere : Détermination des produits, résultats ou impacts des activités d'une intervention après l'exécution des activités [évaluation ex post), avant l'exécution des activités [évaluation ex ante], ou en cours d'activités [évaluation in itinere] ; ces évaluations peuvent être extérieures, et donc réalisées par un tiers acteur, ou participatives, ce qui implique une participation des acteurs engagés dans les activités.

Focus group [ou groupe d'intérêt] : Groupe de discussion généralement mis en place dans une démarche de recherche ou dans un projet de transformation, qui rassemble des individus appartenant à un même groupe social ou confrontés à une même situation, afın de déterminer la position de ce groupe au regard d'un problème, de propositions d'actions ou de l'élaboration d'innovations.

Grappe de projets : Ensemble des projets de recherche, de recherche-développement et de développement, mais aussi des interventions non formalisées en projets, liés à une innovation. C'est donc l'ensemble des projets et d'initiatives passés et en cours, contribuant à une trajectoire d'innovation.

Impacts : Effets à long terme, positifs et négatifs, intentionnels ou non, directs ou indirects, induits par une intervention. Les impacts, c'est ce qui reste une fois que l'intervention est terminée. II peut s'agir d'impacts de différentes natures : économiques, sociaux, territoriaux, environnementaux, politiques, sanitaires. Ils sont mesurés par des indicateurs.

Impacts de $1^{\text {er }}$ niveau : Ce sont les impacts sur les acteurs interagissant directement ou indirectement avec l'équipe en charge d'une intervention.

Impacts de $2^{\mathrm{e}}$ niveau : Ce sont les effets de spillover (impacts indirects), ou les effets de changement d'échelle (horizontal / scaling out et vertical / scaling up]. 
Innovation : Elle peut être définie, au niveau de l'entrepreneur, comme un nouveau produit, un nouveau procédé, une nouvelle manière d'accéder à des services, une nouvelle manière de commercialiser ses produits ou ses services. De manière très générale, l'innovation peut aussi être définie comme une idée mise en œuvre par des acteurs qui ont des intentions de changement ( $c f$. la définition de l'Union européenne dans le partenariat européen pour l'innovation). Elle implique toujours une combinaison de nouvelles techniques ou pratiques (hardware), de nouvelles connaissances et façons de penser [software], de nouvelles organisations et institutions [orgware). Le terme " innovation " peut aussi bien se référer selon les contextes au processus ayant permis de développer une innovation (voir ci-dessous) qu'au produit de ce processus.

Intervention - Une intervention dans ce document définit soit un projet, soit un programme, soit une grappe de projets, la démarche ImpresS ex ante étant applicable à différents " grains » de projets, mais aussi à différentes interventions de recherche ou de développement impliquant la recherche.

Inputs ou " ressources ": Ensemble des moyens et ressources permettant de mener des activités dans une intervention (ressources humaines et matérielles, budget de recherche, informations, connaissances scientifiques ou tacites préexistantes, autres connaissances, etc.) et donc de produire les outputs de l'intervention.

Outcome ou " résultat " : Il est l'appropriation d'un produit de la recherche ou de l'intervention par les acteurs interagissant directement ou indirectement avec la recherche, qui conduit à des changements de pratiques [agricoles ou managériales], des changements organisationnels, d'interactions ou de règles.

Output ou " produit " : Il consiste en la production issue de l'intervention, incluant ce qui n'est pas issu directement de la recherche si l'intervention n'est pas purement une intervention de recherche. Il peut s'agir de connaissance, scientifique ou non (publication, rapport, base de données, méthode, etc.), de formations professionnelles ou académiques, d'une expertise, d'une technologie, d'un réseau ou d'autres formes de production. Les outputs peuvent contribuer à l'émergence d'innovation lorsqu'ils font l'objet d'une appropriation par les acteurs de la société. Dans la méthode décrite dans ce guide, les produits de la recherche [connaissances, prototypes, etc.) élaborés avant le démarrage de l'étude de cas sont considérés comme des inputs alors que ceux élaborés pendant la période de l'étude de cas sont considérés comme des outputs.

Partenaire : C'est un acteur qui apporte un investissement intellectuel, matériel, humain, ou financier substantiel. Un partenaire participe formellement à l'intervention car celle-ci entre dans sa stratégie d'action et son mandat en tant qu'organisation ou institution.

Processus d'innovation : Processus complexe, interactif, parfois tourbillonnaire et imprévisible, très influencé par son environnement, dont le pilotage peut être incertain, voire impossible. Il comporte des phases d'accélération, de ralentissement, et de crise, et implique de nombreux allers-retours entre actions de recherche et actions entreprises par les partenaires des chercheurs, jusqu'à parvenir à la mise en œuvre des innovations par les utilisateurs finaux.

Radar: Représentation graphique qui synthétise les données relatives aux différents impacts identifiés. La méthode ImpresS propose de représenter les impacts après les avoir regroupés en 11 « domaines d'impacts".

Renforcement des capacités : Actions développées par un tiers acteur visant à permettre l'acquisition de nouvelles capacités ou le développement de capacités préexistantes pour les acteurs engagés dans l'innovation.

Situation d'apprentissage : Ensemble de conditions et de circonstances susceptibles d'amener une personne à construire des connaissances, à appliquer et transformer en savoirs et compétences des connaissances. Une telle situation peut être fortuite ou systématique, organisée ou informelle. Elle agit sur l'apprenant en l'interpellant par une observation, une rencontre, un évènement qui pose problème et remet en question ses représentations. Dans ces situations, l'apprentissage est rendu possible par une activité.

Trajectoire d'innovation : Courbe résumant la grappe de projets reliés à une innovation. Une trajectoire d'innovation est le résultat des choix du passé qui déterminent le futur d'une innovation. 


\section{Références}

Altamirano J.A., 2015. Teoria del cambio. Buscando el futuro del territorio con organizaciones en alianza. Informe, Programa Humid Tropics, Jinotega, Nicaragua.

Alvarez S., Jost C.C., Schuetz T., Förch W., Schubert C., Kristjanson P., 2014. Lessons in Theory of Change from the introductory training on Theories of Change, Impact Pathways and Monitoring \& Evaluation, CCSL Learning Brief 10, 4 p., https://cgspace.cgiar.org/handle/10568/52992.

Chevalier J.M.,BucklesD.J.,2008.SAS2 :Guide surlarecherchecollaborativeetl'engagementsocialESKA,CRDI, 328 p., https://www.idrc.ca/fr/book/sas2-guide-sur-la-recherche-collaborative-et-lengagement-social.

Devaux-Spatarakis A., Barret D., Bouyer J., Cerdan C., Dabat M-H., Faure G., Ferré T., Hainzelin E., Medah I., Temple L., Triomphe B., 2016. How Can International Agricultural Research Better Contribute to Innovations: Lessons from Impact Pathways Analysis. In Communication to $12^{\text {th }}$ European IFSA Symposium "Social and Technological Transformation of Farming Systems: Diverging and Converging Pathways," 14. IFSA, http://agritrop.cirad.fr/582679/1/IFSAlessons form impact pathways analysis CIRAD.pdf.

Douthwaite, B.; Alvarez, S.; Cook, S.; Davies, R.; George, Pamela; Howell, J.; Mackay, R.; Rubiano, J. 2007. Participatory impact pathways analysis: a practical application of program theory in research-for-development. Canadian Journal of Program Evaluation, 22[2]:127-159.

Douthwaite B., Mur R., Audouin S., Wopereis M., Hellin J., Moussa A.S., Karbo N., Kasten W., Bouyer J., 2017. Agricultural Research for Development to Intervene Effectively in Complex Systems and the Implications for Research Organizations. Working Paper 12, Royal Tropical Institute, Amsterdam, 20 p.

Durland M.A., Fredericks K.A., 2005. An introduction to social network analysis. New Directions for Evaluation, 107, 5-13.

Earl S., Carden F., Smutylo T., 2001. Outcome Mapping: Building Learning and Reflection into Development Programs, International Development Research Centre, Ottawa, 120 p.

Faure G., Gasselin P., Triomphe B., Temple L., Hocdé H., 2010. Innover avec les acteurs du monde rural : la recherche-action en partenariat, Quæ, Versailles, $224 \mathrm{p}$.

Faure G., Barret B., Dabat M.H., Devaux-Spatarakis A., Hainzelin E., Le Guerroué J.L., Marquié C., 2018. A Participatory Methodology to Assess the Contribution of Agricultural Research to Societal Changes in Developing Countries. World Development, submitted.

Hainzelin E., Barret D., Faure G., Triomphe B., Dabat M-H., 2017. Recherche agricole dans les pays du Sud: dépasser la promesse de l'impact pour piloter la recherche. Perspective 42, Cirad, Montpellier, $4 \mathrm{p}$.

Jost C.C., Alvarez S., Schuetz T., 2014. CCAFS Theory of Change Facilitation Guide. CGIAR Research Program on Climate Change, Agriculture and Food Security (CCAFS), Copenhagen, Denmark.

Lennie J., Tacchi J., Koirala B., Wilmore M., Skuse A., 2011. Equal access participatory monitoring and evaluation toolkit, http://www.betterevaluation.org/en/toolkits/equal_access_participatory_monitoring.

Mayers J., 2005. Stakeholder Power Analysis, Power Tools, International Institute for Environment and Development, London, 24 p., http://www.policy-powertools.org/Tools/Understanding/docs/stakeholder_ power_tool_english.pdf.

Mayne J., 2015. Useful Theory of Change Models. Canadian Journal of Program Evaluation, 30 (2), 119-42, doi:10.3138/cjpe.230.

Morgan P., 1998. Capacity and Capacity Development - Some Strategies. Note prepared for the Political and Social Policies Division, CIDA Policy Branch, Hull, Quebec, 13 p.

Schiffer E., Hauck J., 2010. Net-map: Collecting social network data and facilitating network learning through participatory influence network mapping. Field Method, 22 [3], 231-249.

Temple L., Barret D., Blundo Canto G., Dabat M-H., Devaux-Spatarakis A., Faure G., Hainzelin E., Mathé S., Toillier A., Triomphe B., 2018. Assessing Impacts of Agricultural Research for Development: a systemic model focusing on outcomes. «Forthcoming» in Research Evaluation (in press).

Toillier A., 2012. Contributions méthodologiques à l'évaluation de l'impact de la recherche menée au Cirad. Cas du CEF au Burkina Faso, Document technique et de recherche, Cirad, Montpellier, $26 \mathrm{p}$. 


\section{Annexe. Complémentarités et intégration avec la démarche projet et les outils classiques}

La démarche ImpresS mobilise différents outils existants, en particulier ceux mobilisés par la Délégation à la Valorisation de la Recherche, pour le montage des projets. La démarche ex ante intègre toutes les étapes de la démarche projet des chargés de valorisation. La figure 19 montre la logique de cycle de vie d'un projet et l'articulation avec les étapes de la démarche ImpresS ex ante.

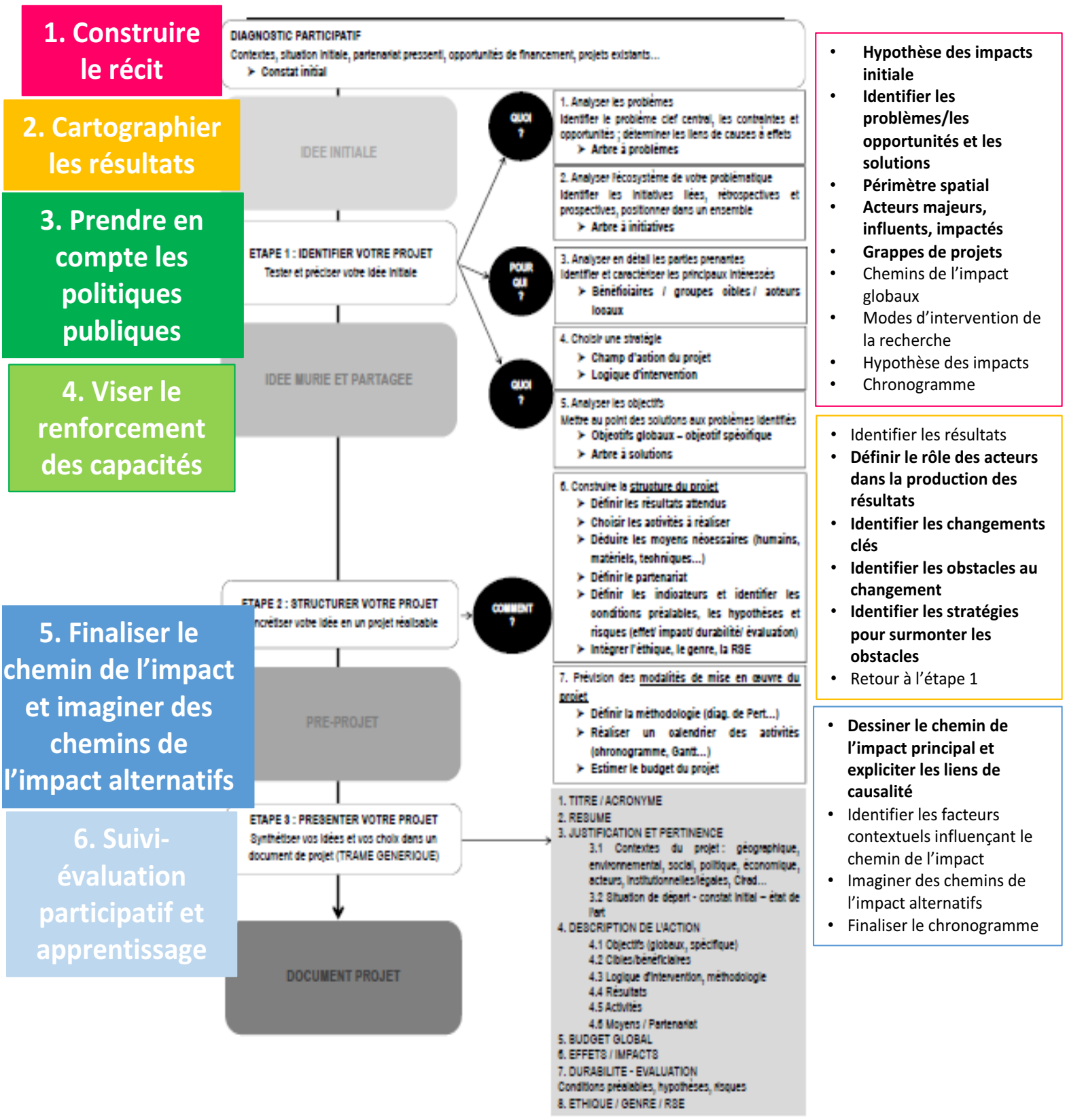

Figure 19 : Articulations entre démarche projet et démarche ImpresS ex ante. 





\section{e) impresS}

IMPACT OF RESEARCH IN THE SOUTF-

https://impress-impact-recherche.cirad.fr/ 